\title{
A SEMI-ANALYTIC SOLUTION FOR FLOW IN FINITE-CONDUCTIVITY VERTICAL FRACTURES USING FRACTAL THEORY
}

\author{
A Thesis \\ by \\ MANUEL COSSIO SANTIZO \\ Submitted to the Office of Graduate Studies of \\ Texas A\&M University \\ in partial fulfillment of the requirements for the degree of \\ MASTER OF SCIENCE
}

August 2012

Major Subject: Petroleum Engineering 
A Semi-Analytic Solution for Flow in Finite-Conductivity Vertical Fractures using Fractal Theory Copyright 2012 Manuel Cossio Santizo 


\title{
A SEMI-ANALYTIC SOLUTION FOR FLOW IN FINITE-CONDUCTIVITY VERTICAL FRACTURES USING FRACTAL THEORY
}

\author{
A Thesis \\ by \\ MANUEL COSSIO SANTIZO \\ Submitted to the Office of Graduate Studies of \\ Texas A\&M University \\ in partial fulfillment of the requirements for the degree of \\ MASTER OF SCIENCE
}

Approved by:

Co-Chairs of Committee, Thomas A. Blasingame

George J. Moridis

$\begin{array}{ll}\text { Committee Members, } & \text { Walter B. Ayers } \\ & \text { Maria A. Barrufet } \\ \text { Head of Department, } & \text { A. Daniel Hill }\end{array}$

$\begin{array}{ll}\text { Committee Members, } & \text { Walter B. Ayers } \\ & \text { Maria A. Barrufet } \\ \text { Head of Department, } & \text { A. Daniel Hill }\end{array}$

August 2012

Major Subject: Petroleum Engineering 


\author{
ABSTRACT \\ A Semi-Analytic Solution for Flow in Finite-Conductivity \\ Vertical Fractures using Fractal Theory. (August 2012) \\ Manuel Cossio Santizo, \\ M.Eng., Imperial College London \\ Co-Chairs of Advisory Committee: Dr. Thomas A. Blasingame \\ Dr. George J. Moridis
}

The exploitation of unconventional reservoirs goes hand in hand with the practice of hydraulic fracturing and, with an ever increasing demand in energy, this practice is set to experience significant growth in the coming years. Sophisticated analytic models are needed to accurately describe fluid flow in a hydraulic fracture and the problem has been approached from different directions in the past 3 decades — starting with the use of line-source functions for the infinite conductivity case, followed by the application of Laplace Transforms and the Boundary-Element Method for the finite-conductivity case. This topic remains an active area of research and, for the more complicated physical scenarios such as multiple transverse fractures in ultra-tight reservoirs, answers are presently being sought.

Fractal theory has been successfully applied to pressure transient testing, albeit with an emphasis on the effects of natural fractures in pressure-rate behavior. In this work, we begin by performing a rigorous analytical and numerical study of the Fractal Diffusivity Equation and we show that it is more fundamental than the classic linear and radial diffusivity equations. Subsequently, we combine the Fractal Diffusivity Equation with the Trilinear Flow Model, culminating in a new semi-analytic solution for flow in a finite-conductivity vertical fracture which we name the "Fractal-Fracture Solution". This new solution is instantaneous and has an overall accuracy of $99.7 \%$, thus making it comparable to the Trilinear Pseudoradial Solution for practical purposes. It may be used for pressure transient testing and reservoir characterization of hydrocarbon reservoirs being produced by a vertically fractured well. Additionally, this is the first time that fractal theory is used in fluid flow in porous media to address a problem not related to 
reservoir heterogeneity. Ultimately, this work is a demonstration of the untapped potential of fractal theory; our approach is very flexible and we believe that the same methodology may be extended to develop new reservoir flow solutions for pressing problems that the industry currently faces. 


\section{DEDICATION}

This thesis is dedicated to my parents, Fernando and Rosa. The effort that went into this thesis is a reflection of your unconditional love and support.

In any field, find the strangest thing and then explore it. - John Archibald Wheeler

No man should escape our universities without knowing how little he knows. - J. Robert Oppenheimer 


\section{ACKNOWLEDGEMENTS}

It is difficult for me to imagine a more privileged set of circumstances under which to do one's graduate studies. The fact of the matter is that for the past 2 years I have been paid to learn from some of the brightest minds in petroleum engineering. I would like to thank Drs. Thomas A. Blasingame and George J. Moridis for believing in me, welcoming me in their research group and for funding my studies. Wherever my career takes me in the future, I will always hold them in the highest esteem.

I would also like to acknowledge all my fellow students in Texas A\&M University for their support, friendship and for making my stay in College Station a most enjoyable experience.

Finally, my appreciation goes to the faculty of the department, for dedicating their lives to academia, for all the knowledge they have shared, and for the wonderful classes that I attended. 


\section{TABLE OF CONTENTS}

\section{Page}

ABSTRACT .iii

DEDICATION $\mathrm{V}$

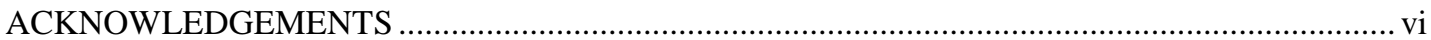

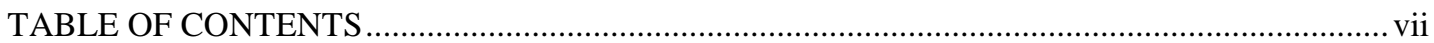

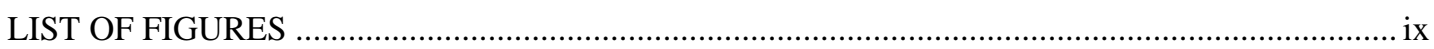

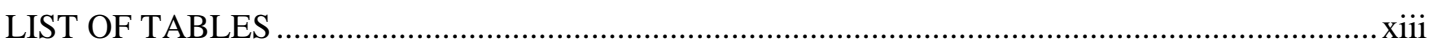

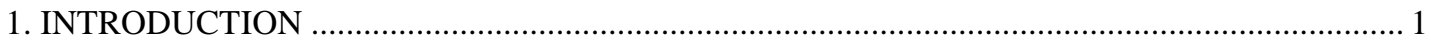

1.1 Statement of the Problem ................................................................................................. 1

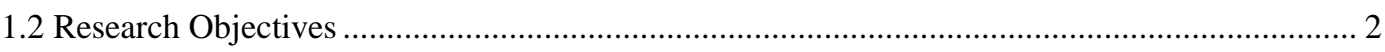

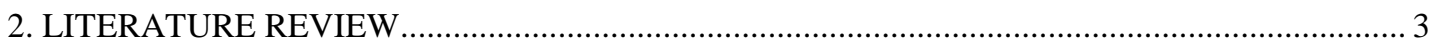

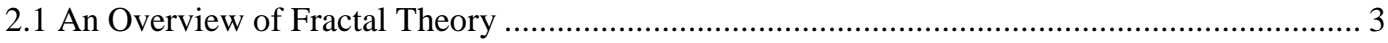

2.2 Fractal Theory Applied to Fluid Flow in Porous Media .................................................. 7

2.3 Fractals in Reservoir Engineering ......................................................................... 9

2.4 Reservoir Engineering Problems Related to Tight Gas/Shale Gas Reservoirs...................... 14

3. A STUDY OF THE FRACTAL DIFFUSIVITY EQUATION............................................ 16

3.1 Derivation of the Fractal Diffusivity Equation ......................................................... 16

3.2 Discussion of the Fractal Porosity Permeability Relations............................................. 24

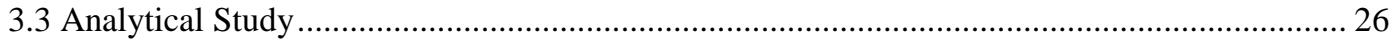

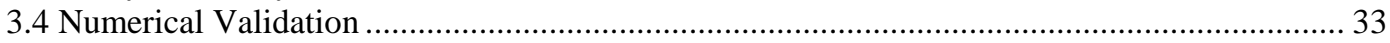

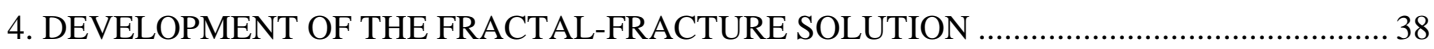

4.1 Trilinear Flow Model with Fractal Modification .............................................................. 38

4.2 Analytic Derivation of the Open-Ended Fractal-Fracture Solution .................................... 41

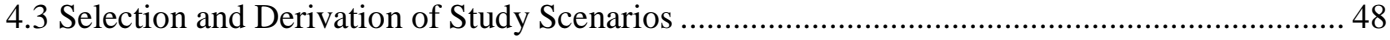

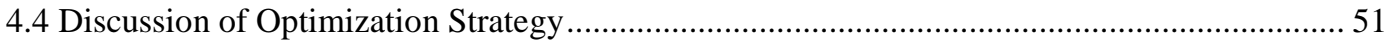

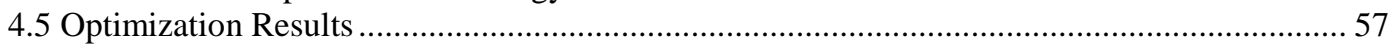




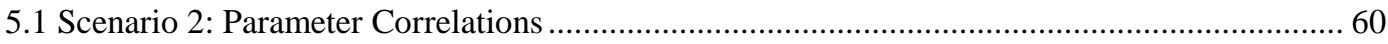

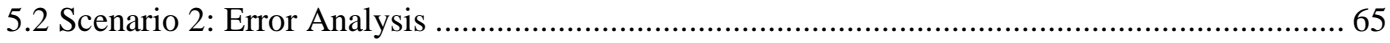

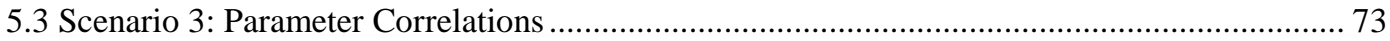

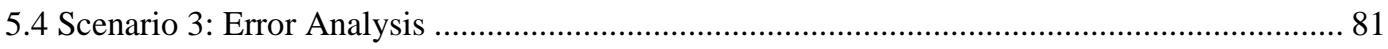

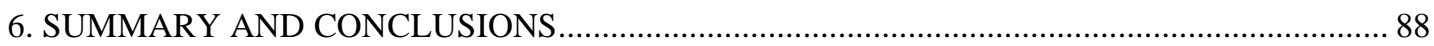

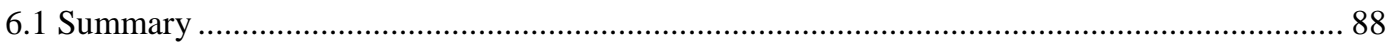

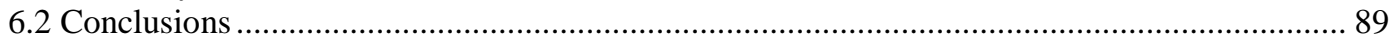

6.3 Recommendations for Future Research...................................................................... 89

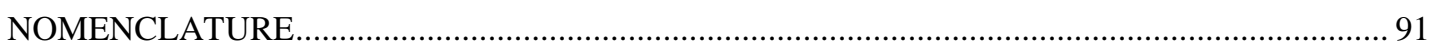

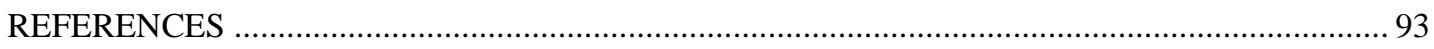

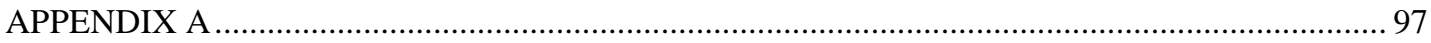

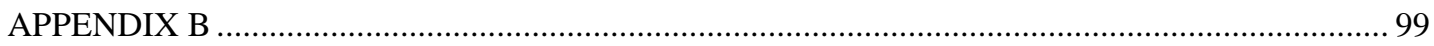

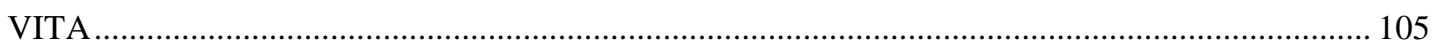




\section{LIST OF FIGURES}

Figure 1.1 - U.S. natural gas production, 1990-2035, trillion cubic feet per year (US DOE EIA 2011)

Figure 2.1 — The triadic Koch curve (Feder 1988) ....................................................................

Figure 2.2 - The coast of Lake Mead (Moran 2010). The "coastline paradox" is the counterintuitive observation that the coastline of a landmass does not have a welldefined length. This results from the fractal-like properties of coastlines. It was first observed by Lewis Fry Richardson (Mandelbrot 1967)............................................ 7

Figure 2.3 — Detail of a Romanesco broccoli (McNally 2010) ..................................................... 8

Figure 2.4 - Schematic of a pore fractal (Ruis 2008). Both the pore surface (in red) and pore space (in white) have been shown to exhibit fractal properties with respect to pore size (Yu 2008).

Figure 2.5 - Injection of a low viscosity fluid in a high viscosity fluid. (a) Air displacing glycerol. (b) Water displacing a non-Newtonian high viscosity mixture of sclerogutan in water (Feder 1988). This phenomenon is known as viscous fingering and it has been shown experimentally to possess fractal properties (Maloy et al. 1987).

Figure 2.6 - Schematic of a fractal fracture network embedded in a Euclidean matrix (Chang and Yortsos 1990)

Figure 2.7 — Radial Composite Model with Fractal Transition Zone (Fuentes-Cruz et al. 2010)

Figure 3.1 - Schematic of the equivalency between fractal linear flow and classic radial flow. Even though the top and bottom cases describe different physical scenarios, the Fractal Diffusivity Equation predicts that both should yield the same pressure signal at the wellbore. This has been verified analytically and numerically in this thesis....

Figure 3.2 - Mathematica 8 code to solve Equ 3.46

Figure 3.3 - Schematic of the 1D linear reservoir common to all cases. This reservoir has 1000 gridblocks in the $\mathrm{x}$-direction, each with its own porosity and permeability, as stipulated in the FPPR. The permeability and porosity distributions with respect to $x$, for all 8 cases, is shown in Figure 3.4 and Figure 3.5, respectively

Figure 3.4 - Permeability vs. distance. The linear 1D reservoir shown in Figure 3.3 has a permeability that varies with distance from the wellbore, as stipulated by the FPPR (Eq. 3.1). The 8 cases that were simulated are listed in Table 3.1 are labeled accordingly.

Figure 3.5 - Porosity vs. distance. The linear 1D reservoir shown in Figure 3.3 has a porosity that varies with distance from the wellbore, as stipulated by the FPPR (Eq. 3.2). The 8 cases that were simulated are listed in Table 3.1 are labeled accordingly. 
Figure 3.6 - Comparison between analytical and numerical results of the Fractal Diffusivity Equation. Note that Case 1 is equivalent to a Euclidean linear case, and Case 5 is equivalent to a Euclidean radial case. The excellent agreement in Case 5 validates numerically the equivalency proposed in Figure 3.1.

Figure 4.1 - Schematic of the trilinear flow concept as proposed by Lee and Brockenbrough (1986). Because of symmetry only a quadrant of the flow domain is considered. Region 3 flows in the $\mathrm{x}$ direction and meets Region 2 at the dashed lines at $x=x_{f}$; both Regions 2 and 3 involve formation flow. Region 2 flows in the y-direction and meets Region 1 at the dashed lines at $y=b_{f}$; Region 1 represents an idealized vertical fracture. Finally, the fracture flow of Region 1 feeds the wellbore, represented by the circle.

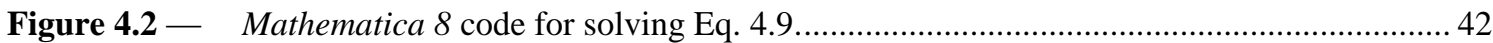

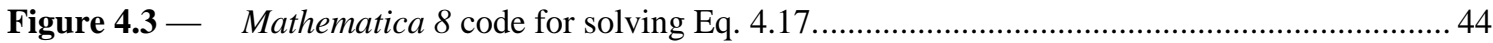

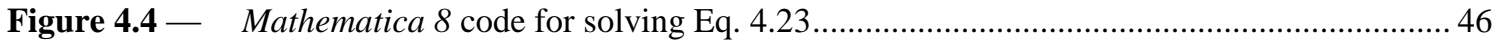

Figure 4.5 - Optimization process of the fractal parameters in the FFS. We begin with an initial guess of the values of the unknown fractal parameters, compute the Fractal-Fracture Solution with them, compare the results to the Cinco-Meng Solution, and attempt to optimize the parameter value by minimizing an objective function. The optimization is terminated when the Levenberg-Marquardt algorithm determines that the objective function $O_{F}$ cannot be further minimized.

Figure 4.6 - Evaluation of five different objective functions for a low conductivity $\left(F_{c D}=0.5\right)$ case

Figure 4.7 - Evaluation of five different objective functions for a high conductivity $\left(F_{c D}=10^{4}\right)$ case.

Figure 4.8 - The Fractal-Fracture Solution was derived analytically for the three Scenarios considered in Table 4.1. The solutions of Table 4.2 were each numerically optimized to match the Cinco-Meng (1988) solution. The $\mathrm{L}^{1}$ relative error norm (in percent) is plotted against fracture conductivity for each of these scenarios.

Figure 4.9 - Values of optimized fractal parameters versus the dimensionless fracture conductivity $\left(F_{c D}\right)$. The Fractal-Fracture Solution (Scenario 2) was numerically optimized to obtain values for the 4 fractal parameters in order to match the Cinco-Meng (1988) solution. Note that all curves are well-behaved and should lend themselves to approximations by smooth, closed form functions.

Figure 4.10 - Numerically optimized fractal parameters for Scenario 3 ( $d$-parameters)....................... 59

Figure 4.11 - Numerically optimized fractal parameters for Scenario 3 ( $\theta$-parameters).......................59 
Figure 5.1 - Parameter $\theta_{2}$ versus dimensionless fracture conductivity $F_{c D}$. Each black circle represents the optimized $\theta_{2}$ for a given $F_{c D}$ value. The red line is the model

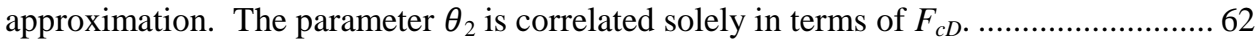

Figure 5.2 - Parameter $d_{2}$ versus dimensionless fracture conductivity $F_{c D}$. Each black circle represents the optimized $d_{2}$ for a given $F_{c D}$ value. The red line is the model approximation. The parameter $d_{2}$ is correlated solely in terms of $F_{c D} \ldots \ldots \ldots \ldots \ldots \ldots \ldots \ldots . . . \ldots 2$

Figure 5.3 - Parameter $d_{3}$ versus parameter $d_{2}$. Each black circle represents the optimized $d_{3}$ value compared to a corresponding optimized $d_{2}$ value. The red line is the model approximation. In this rendering, the parameter $d_{3}$ is correlated solely in terms of the parameter $d_{2}$ using a quadratic trend - which seems appropriate given the trending of these data.

Figure 5.4 - Parameter $\theta_{3}$ versus parameter $d_{3}$. Each black circle represents the optimized $\theta_{3}$ value compared to a corresponding optimized $d_{3}$ value. The red line is the model approximation. . In this rendering, the parameter $\theta_{3}$ is correlated solely in terms of the parameter $d_{3}$ using a linear trend - which seems appropriate given the trending of these data.

Figure 5.5 - (Scenario 2) Log-log plot of dimensionless pressure function versus dimensionless time for the FFS and Cinco-Meng Solutions (1988).

Figure 5.6 - (Scenario 2) Semi-log plot of dimensionless pressure versus dimensionless time for the FFS and Cinco-Meng (1988) Solutions.

Figure 5.7 - (Scenario 2) Relative error (percent) in the dimensionless pressure solutions versus dimensionless time.

Figure 5.8 - (Scenario 2) $\mathrm{L}^{1}$ relative error norms for the dimensionless pressure solutions for the closed form Fractal-Fracture Solution (FFS) and the Trilinear Pseudoradial Solution (TPRS) (Blasingame and Poe 1993) versus the dimensionless fracture conductivity $\left(F_{c D}\right)$. Reference solution obtained from Cinco-Meng (1988).

Figure 5.9 - (Scenario 2) Log-log plot of dimensionless pressure derivative function versus dimensionless time for the FFS and Cinco-Meng Solutions (1988).

Figure 5.10 - (Scenario 2) Semi-log plot of dimensionless pressure derivative versus dimensionless time for the FFS and Cinco-Meng Solutions (1988).

Figure 5.11 - (Scenario 2) Relative error (percent) in the dimensionless pressure derivative solutions versus dimensionless time.

Figure 5.12 - (Scenario 2) $\mathrm{L}^{1}$ relative error norms for the dimensionless pressure derivative solutions for the closed form Fractal-Fracture Solution (FFS) and the Trilinear Pseudoradial Solution (TPRS) (Blasingame and Poe 1993) versus the dimensionless fracture conductivity $\left(F_{c D}\right)$. Reference solution obtained from Cinco-Meng (1988). 
Figure 5.13 - Parameter $\theta_{1}$ versus dimensionless fracture conductivity $F_{c D}$. Each black circle represents the optimized $\theta_{1}$ for a given $F_{c D}$ value. The red line is the model

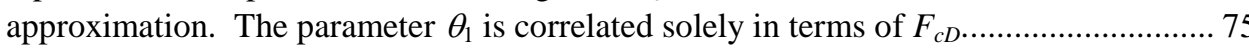

Figure 5.14 - Parameter $d_{3}$ versus dimensionless fracture conductivity $F_{c D}$. Each black circle represents the optimized $d_{3}$ for a given $F_{c D}$ value. The blue line is the model approximation. The parameter $d_{3}$ is correlated solely in terms of $F_{c D}$.

Figure 5.15 - Parameter $\theta_{3}$ versus parameter $d_{3}$. Each black circle represents the optimized $\theta_{3}$ for a given $d_{3}$ value. The red line is the model approximation. The parameter $\theta_{3}$ is correlated solely in terms of $d_{3}$

Figure 5.16 - Parameter $d_{1}$ versus dimensionless fracture conductivity $F_{c D}$. Each black circle represents the optimized $d_{1}$ for a given $F_{c D}$ value. The red line is the model approximation. The parameter $d_{1}$ is correlated solely in terms of $F_{c D}$.

Figure 5.17 - Parameter $\theta_{2}$ versus parameter $d_{1}$. Each black circle represents the optimized $\theta_{2}$ for a given $d_{1}$ value. The red line is the model approximation. The parameter $\theta_{2}$ is correlated solely in terms of $d_{1}$.....

Figure 5.18 - Parameter $d_{2}$ versus parameter $d_{1}$. Each black circle represents the optimized $d_{2}$ for a given $d_{1}$ value. The red line is the model approximation. The parameter $d_{2}$ is correlated solely in terms of $d_{1}$.

Figure 5.19 - (Scenario 3) Log-log plot of dimensionless pressure function versus dimensionless time for the FFS and Cinco-Meng Solutions (1988).

Figure 5.20 - (Scenario 3) Semi-log plot of dimensionless pressure versus dimensionless time for the FFS and Cinco-Meng Solutions (1988).

Figure 5.21 - (Scenario 3) Relative error (percent) in the dimensionless pressure solutions versus dimensionless time.

Figure 5.22 - (Scenario 3) $\mathrm{L}^{1}$ relative error norms for the dimensionless pressure solutions for the closed form Fractal-Fracture Solution (FFS) and the Trilinear Pseudoradial Solution (TPRS) (Blasingame and Poe 1993) versus the dimensionless fracture conductivity

Figure 5.23 - (Scenario 3) Log-log plot of dimensionless pressure derivative function versus dimensionless time for the FFS and Cinco-Meng Solutions (1988).

Figure 5.24 - (Scenario 3) Semi-log plot of dimensionless pressure derivative versus dimensionless time for the FFS and Cinco-Meng (1988) Solutions.

Figure 5.25 - (Scenario 3) Relative error (percent) in the dimensionless pressure derivative solutions versus dimensionless time.

Figure 5.26 - (Scenario 3) $\mathrm{L}^{1}$ relative error norms for the dimensionless pressure derivative solutions for the closed form Fractal-Fracture Solution (FFS) and the Trilinear Pseudoradial Solution (TPRS) (Blasingame and Poe 1993) versus the dimensionless fracture conductivity $\left(F_{c D}\right)$. Reference solution obtained from Cinco-Meng (1988). 


\section{LIST OF TABLES}

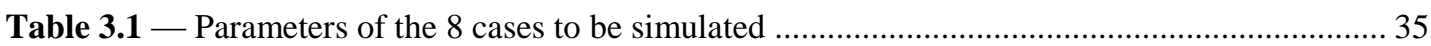

Table 3.2 - Reservoir and flow parameters common to all simulation cases .............................. 35

Table 4.1 - Scenarios to be optimized ............................................................................. 49

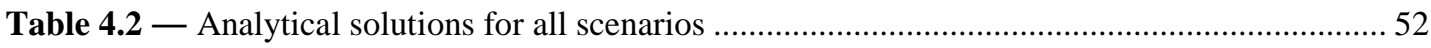

Table 4.3 - Evaluated weight coefficients for objective function ........................................... 55

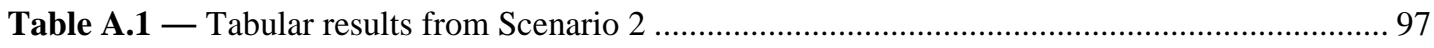

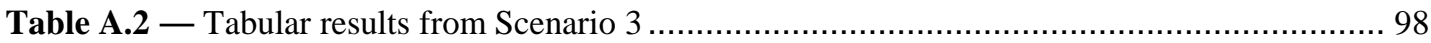




\section{INTRODUCTION}

\subsection{Statement of the Problem}

Unconventional resources are a very promising source of energy, mainly because of their enormous estimated reserves. As shown in Figure 1.1, shale gas, tight gas and coalbed methane are expected to be the main sources of natural gas production in the coming decades. Due to the extremely low permeabilities, it is generally not possible to economically exploit unconventional reservoirs without the use of horizontal drilling and hydraulic fracturing. Officially, a tight gas is defined as having a permeability below $0.1 \mathrm{md}$, but current shale gas/oil can be in the nano-darcy range.

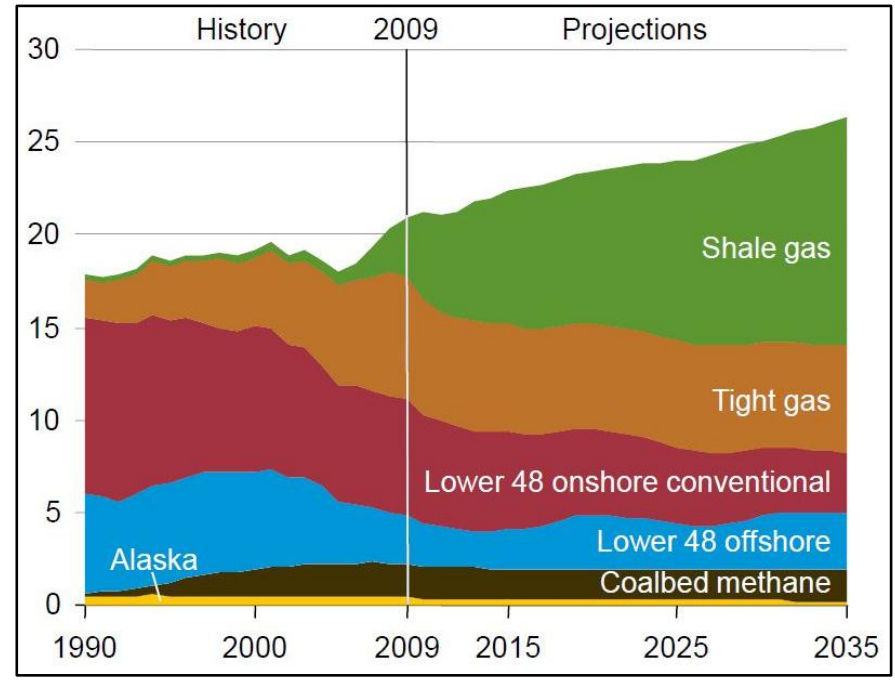

Figure 1.1 - U.S. natural gas production, 1990-2035, trillion cubic feet per year (US DOE EIA 2011)

This thesis follows the style of the SPE Journal. 
Compounding this extremely low permeability is the fact that other factors such as sorption effects, natural fractures, heterogeneity or multiphase flow may play an important role in determining production performance and estimated ultimate recovery. In order to economically exploit a field, engineers must be able to predict, with a high degree of accuracy, the production performance of the reservoir. This will guide the asset team in their expectations of cash flow, and therefore help them decide what investments to make next.

Mathematical tools can be broadly divided into two categories: analytical and numerical. Analytical tools have the advantage that they are very fast and easy to manipulate; but on the other hand, analytical methods may be fairly limited in the types of the physical scenarios that they can model. Numerical models are much more flexible and can accommodate a variety of scenarios, but can be expensive - in terms of computational time and problem set-up.

We believe that new analytical (or perhaps a better term would be semi-analytical) solutions can be created by merging fractal theory with existing reservoir solutions. This thesis utilizes fractal theory and provides the detailed proposal, development, calibration, and validation of the "Fractal-Fracture Solution" (FFS) for the case of a single vertical well containing a single (planar) hydraulic fracture of finite fracture conductivity producing in an infinite-acting homogeneous reservoir.

\subsection{Research Objectives}

The primary objectives of this work are

- To perform a rigorous numerical and analytical study of the Fractal Diffusivity Equation, which is derived in detail. We demonstrate that analytical solutions for various combinations of boundary conditions can be obtained by exploiting the capabilities of symbolic math software.

- To develop an accurate semi-analytical solution for flow in a single finite-conductivity vertical fracture fully penetrating a homogeneous infinite-acting reservoir. To our knowledge, this is the first application of fractal theory for a problem that is not related to heterogeneous systems. 


\section{LITERATURE REVIEW}

\subsection{An Overview of Fractal Theory}

Benoit B. Mandelbrot (1982) generated a widespread interest in fractal geometry — a concept introduced by Mandelbrot himself. His book (Mandelbrot 1982) is the standard reference and contains both the elementary concepts and a broad range of new ideas. Mandelbrot (1982) defined a fractal as a "rough or fragmented geometric shape that can be split into parts, each of which is (at least approximately) a reduced-size copy of the whole".

The complex nature of a phenomenon is manifested in the underlying intricate geometry which in most cases can be described in terms of objects with a non-integer (fractal) dimension (Frame et al. 2012). Put in simpler terms, a property of a given system is said to be fractal if its seemingly chaotic and unpredictable behavior with respect to space or time can be captured in a simple power-law equation. In this equation, which we discuss below, the so-called "fractal dimension" is the only variable and it characterizes the chaotic property of the studied system. Thus, seemingly incomprehensible problems are distilled and encapsulated in the simplicity of a power-law equation.

The fractal dimension has been defined (Feder 1988) as a statistical quantity that gives an indication of how completely a fractal appears to fill a space as one zooms down to finer and finer scales. Mandelbrot (1982) and Feder (1988) discuss at length how to determine the fractal dimension of a geometric object or a data set. Given the highly visual nature of fractals, it is best to illustrate this rather abstract concept via the classic example of the triadic Koch curve (Figure 2.1). 


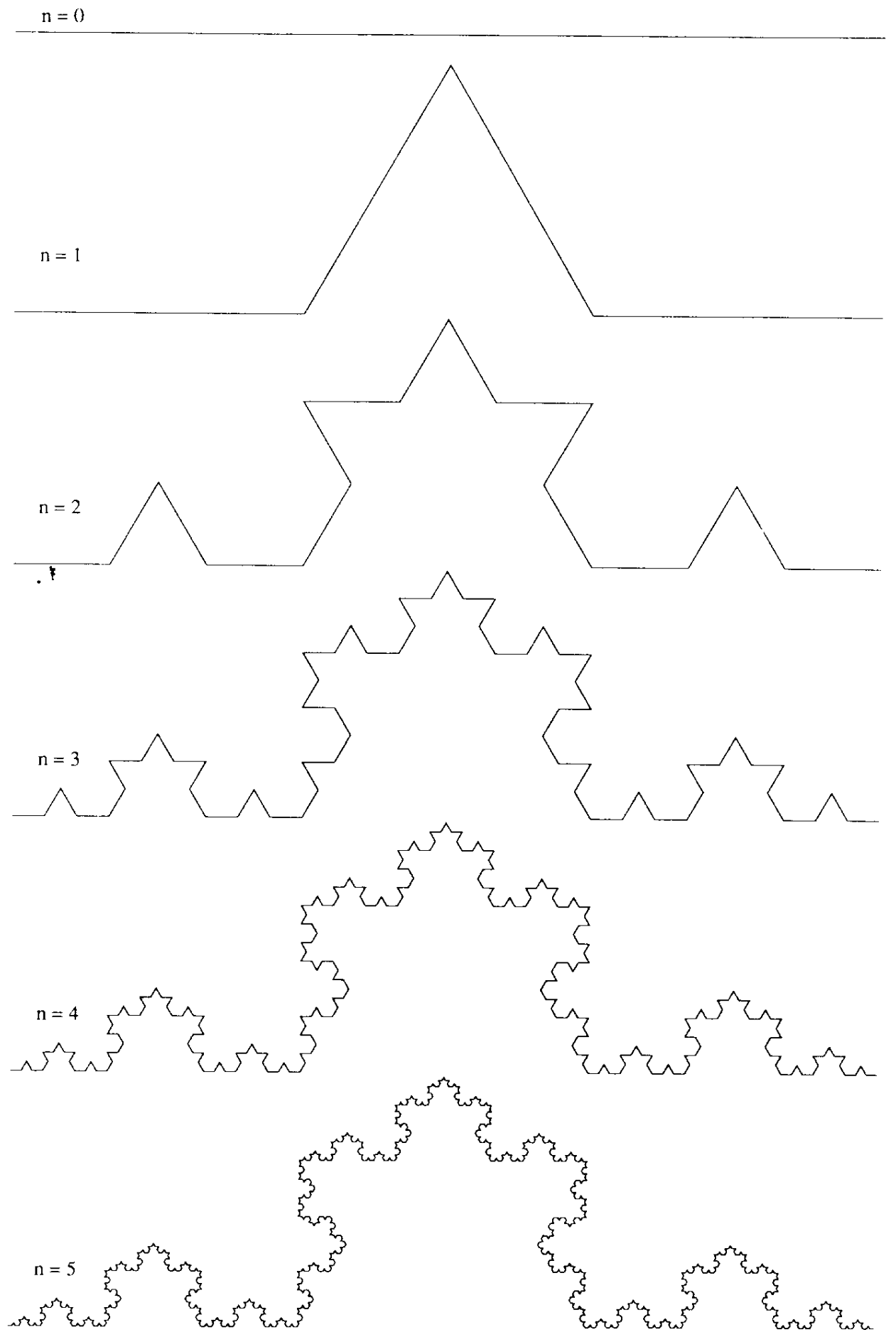

Figure 2.1 $-\quad$ The triadic Koch curve (Feder 1988) 
The construction of the Koch curve starts with a line segment of unit length 1. This starting form is called the initiator and may be replaced by a polygon such as an equilateral triangle, a square or some other polygon. The initiator is the 0 -th generation of the Koch curve. The construction of the Koch curve proceeds by replacing each segment of the initiator by the generator shown as the curve marked $n=1$ in Figure 2.1. Thus we obtain the first generation, which is a curve of 4 line segments each of length $1 / 3$; the length of the curve is now 4/3. The next generation is obtained by replacing each line segment by a scaled-down version of the generator. Thus in the second generation we have a curve consisting of $4 \times 4=$ 16 segments each having $1 / 3 \times 1 / 3=1 / 9$ length; the length of the second generation is therefore $(4 / 3)^{2}=$ 16/9. At the $n$-th generation, the length of the Koch curve will be $(4 / 3)^{\mathrm{n}}$. In general terms for any $n$-th generation, the number of segments is referred to as the property and the length of the segments is referred to as the yardstick.

In order for an object to be fractal, the following formula must hold true for all generations:

$$
\Delta P=\left(\frac{1}{\Delta y}\right)^{\alpha}
$$

Where $\alpha$ is the fractal dimension (dimensionless), $\Delta P$ is the change in the property (dimensionless) and $\Delta y$ is the change in yardstick (dimensionless) as we go from generation $n$ to generation $(n+1)$. In the Koch curve, we saw these values were $\Delta P=4$ and $\Delta y=1 / 3$, respectively. The triadic Koch curve therefore has a fractal dimension of

$$
4=\left(\frac{1}{1 / 3}\right)^{\alpha} \Rightarrow \alpha=\frac{\ln (4)}{\ln (3)}=1.2618
$$

Strictly speaking, what is 'fractal' is not the Koch curve itself, but rather a specific property of the curve. Furthermore, this property needs to be fractal with respect to a reference distance (the yardstick). As already stated, in this example the property on which we focus is the number of segments for a given $n$-th generation. The yardstick we selected is the length of the segments in the same $n$-th generation. For example, in the second generation, there are 16 segments (property) and each segment has a length of $1 / 9$ 
(yardstick). One can easily calculate the property and yardstick at the $n$-th generation without having to do the drawing and manually count each line.

This is the concept at the heart of fractal theory, and there are at least three implications worth mentioning. First of all, one can now see why it is possible to 'zoom-in' indefinitely into a fractal object and always see the same image; the process of creating the $(n+1)$-th generation from the $n$-th generation is always the same. Secondly, as $n$ tends toward infinity, the length of the Koch curve tends toward infinity, even though the length of the segment or yardstick tends toward 0. This is what's known as "The Coastline Paradox" (Mandelbrot 1967) - i.e., the realization that it is impossible to get a precise measurement of the length of a coastline because of its fractal properties. Finally, when the fractal dimension is an integer, we call it Euclidean, and geometrically we will obtain smooth shapes such as a full triangle, smooth differentiable lines, full squares or circles.

Fractals have proved to have considerable flexibility in the types of problems they can address, and they have been successfully used in a variety of unrelated fields, both in and outside the realm of natural sciences. Examples include the classification of histopathology slides in medicine, enzymology, signal and image compression, seismology, soil mechanics, fracture mechanics, generation of patterns for camouflage, analysis of price series and, as we will show, fluid flow in porous media.

Using fractal geometry and scaling as a language in related theoretical, numerical and experimental investigations, it has been possible to gain a deeper insight into previously intractable problems. Among many others, a better understanding of growth phenomena, turbulence, iterative functions, colloidal aggregation, biological pattern formations, stock market behavior, and property distributions in heterogeneous materials has emerged through the application of such concepts as scale invariance, self-affinity and multifractality (Frame et al. 2012). Fractals are abundantly present in nature, from the gigascale (Figure 2.2) to the microscale (Figure 2.3). Ultimately, the power of fractals as an analytical tool lies in its ability to capture elegantly and succinctly the chaotic nature of complex systems, a task for which smooth curves and continuous shapes are ineffective. 


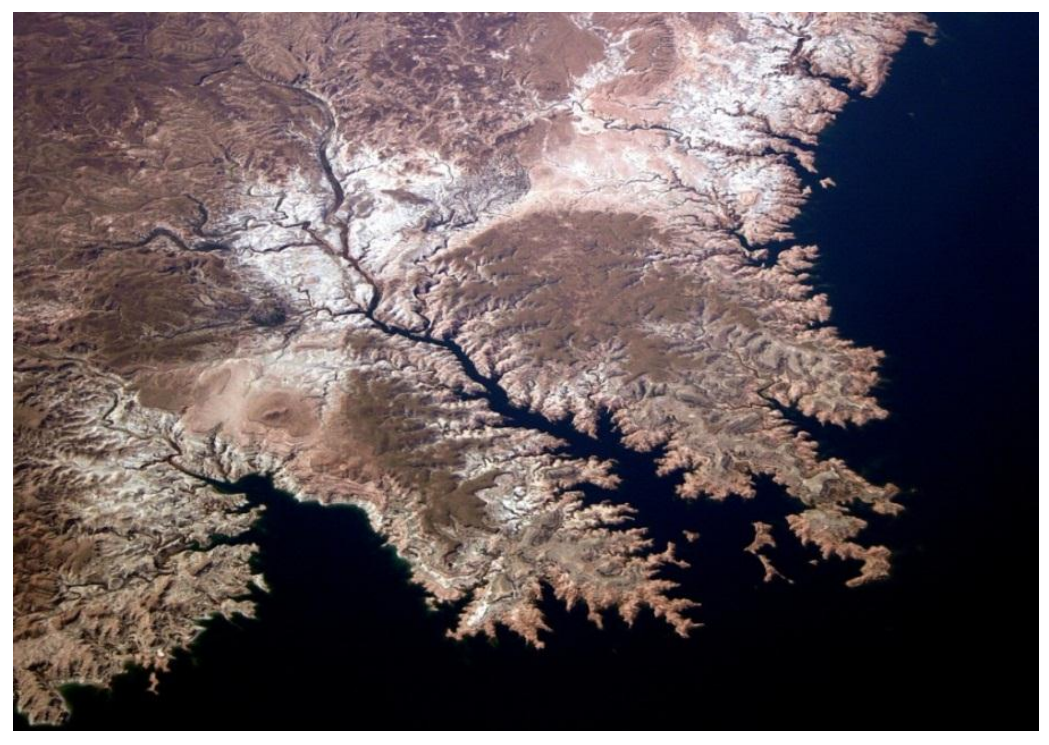

Figure 2.2 - The coast of Lake Mead (Moran 2010). The "coastline paradox" is the counterintuitive observation that the coastline of a landmass does not have a well-defined length. This results from the fractal-like properties of coastlines. It was first observed by Lewis Fry Richardson (Mandelbrot 1967).

\subsection{Fractal Theory Applied to Fluid Flow in Porous Media}

Fractal geometry has been shown to have potential in the analysis of flow and transport properties in porous media. Katz and Thompson (1985) are probably the first investigators to present experimental evidence indicating that the pore spaces of a set of sandstone samples are fractals and self-similar over three to four orders of magnitude in length, extending from $10 \AA$ to $100 \mu \mathrm{m}$. Katz and Thompson argued that the pore volume (Figure 2.4) is a fractal with the same fractal dimension as the pore-rock interface. This conclusion was supported by correctly predicting the porosity from the fractal dimension, which was measured by a log-log plot of the number of pores versus the pore size (Yu 2008). Note that the concept of property vs. yardstick that was discussed earlier is present here, where the number of pores is the property and the pore size is the yardstick.

Krohn and Thompson (1986) carried out measurements on sandstone pores and confirmed their fractal properties by estimating similar fractal dimensions on five different sandstone samples. Smidt and Monro 
(1998) performed experimental investigations on the images of laboratory-made synthetic sandstone. Their results also showed that the pore space of the synthetic sandstone was fractal, with a similar fractal dimension estimated for the different samples of their study.

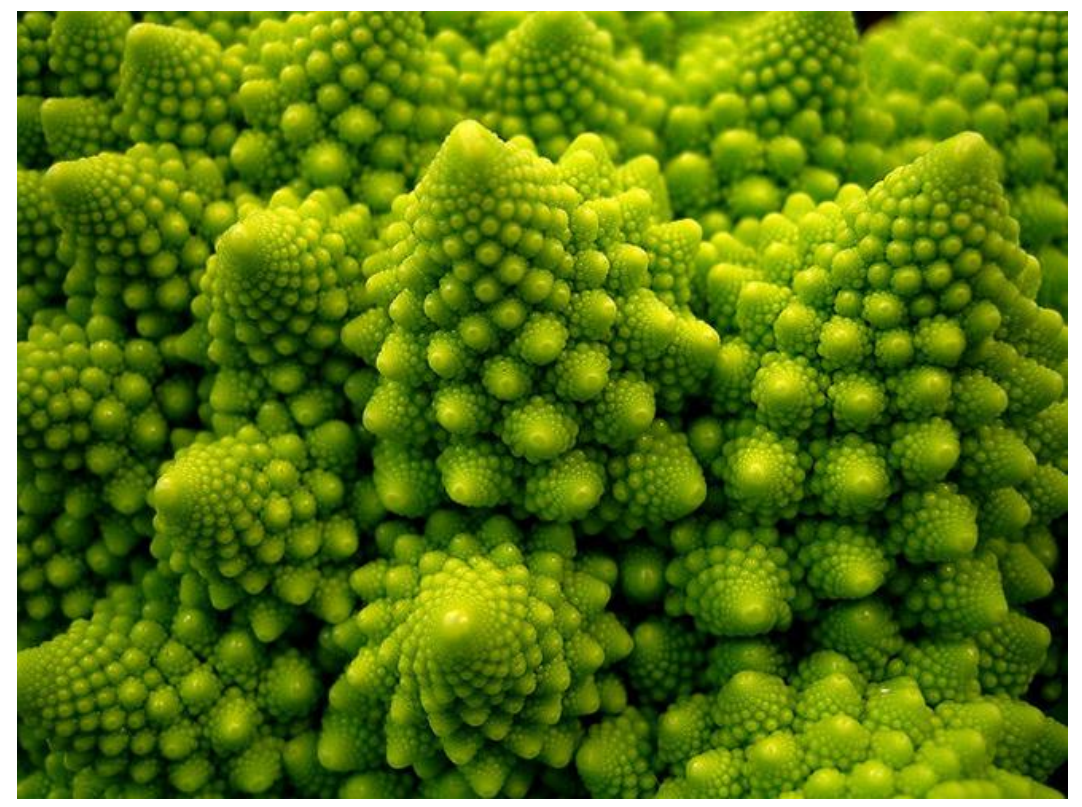

Figure 2.3 - Detail of a Romanesco broccoli (McNally 2010)

So far we have discussed the fractal nature of the arrangement of the pore spaces in a porous medium. In order to complete the discussion, we must also question the nature of the fluid flow through them, which is physically a different problem. In other words, just because one has understood the structure of the pore spaces (porosity) does not mean one can claim the same about the conductance through the pore system (i.e., permeability).

Indeed, the problem of viscous fingering in porous media is of central importance in hydrocarbon recovery. As would be expected, it has been shown that viscous fingering in porous media is also fractal (Maloy et al. 1985). A porous medium is usually defined as a structure that consists of pores with different sizes, and these pores are randomly distributed in solid space. The pores may be connected to 
form tortuous capillaries through which fluids flow. The tortuous flow paths may be similar to the triadic Koch curve (Maloy et al. 1987), which was just discussed. Therefore, in addition to characterizing the geometry of the pore spaces, the tortuous flow paths may need their own fractal dimension, so that one may characterize the convolutedness of the capillary pathways ( $\mathrm{Yu}$ 2008). One example from experimental studies may be found in Figure 2.5, where a low viscosity fluid injected in a high viscosity medium shows a fractal viscous fingering type of displacement (Maloy et al. 1985).

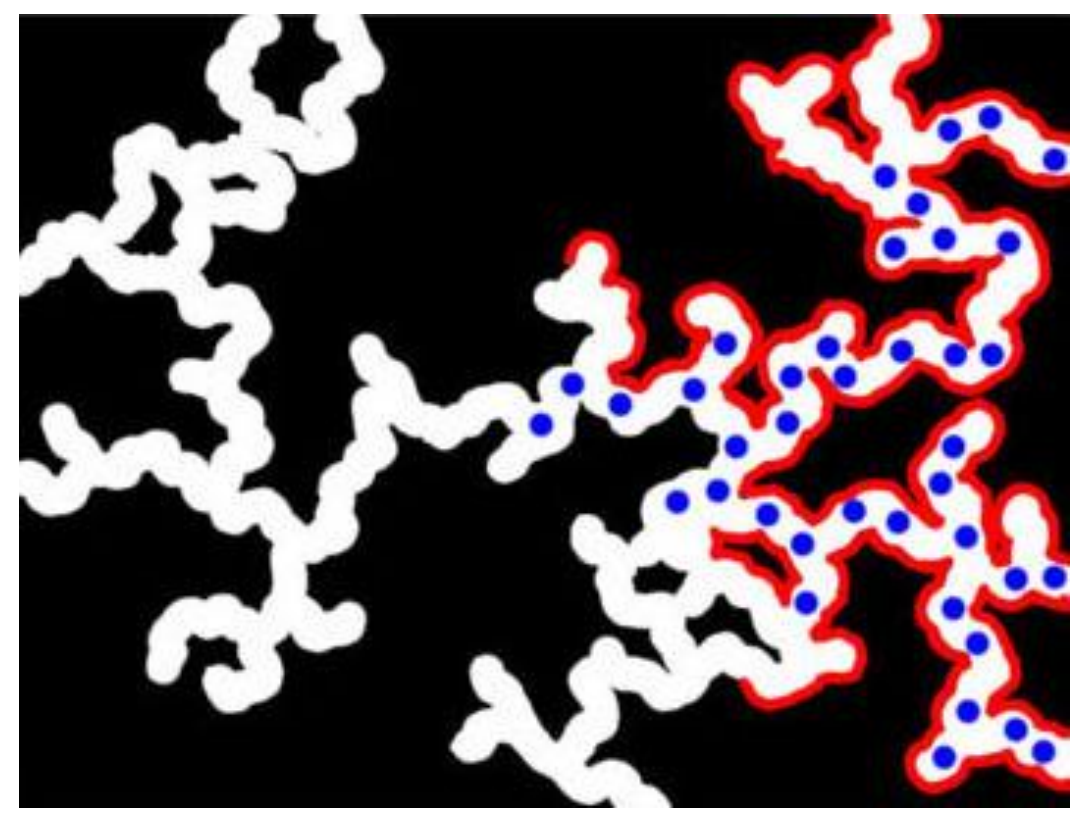

Figure 2.4 - Schematic of a pore fractal (Ruis 2008). Both the pore surface (in red) and pore space (in white) have been shown to exhibit fractal properties with respect to pore size (Yu 2008).

\subsection{Fractals in Reservoir Engineering}

Having discussed fractals in porous media in general terms, we now focus on the practice of reservoir engineering and discuss previous studies in this area. While research in pressure transients of naturallyfractured systems has made important advances, it has been realized that fractal models do not always give satisfactory results (Acuña et al. 1995). Standard models have their underpinnings on the classical notion 
that naturally-fractured systems are characterized by a few distinct scales that delineate the fracture network and the embedded matrix. Variations on this approach, include randomly generated fracture networks, triple-porosity systems (Abdassah and Ershaghi 1986), etc. - and although these approaches add complexity, they still obey the general premise that the network of fractures is dense and space filling; namely, that it is of Euclidean geometry. Instead, it is perhaps more reasonable to expect that what feeds the well in a naturally-fractured system is a network of fractures, which is not necessarily space-filling or perfectly connected. Such networks are best characterized by fractal geometry. The advantages of a fractal geometry description is that it generalizes the underlying geometry in a non-trivial manner and allows for a direct and novel interpretation of responses (Acuña et al. 1995).
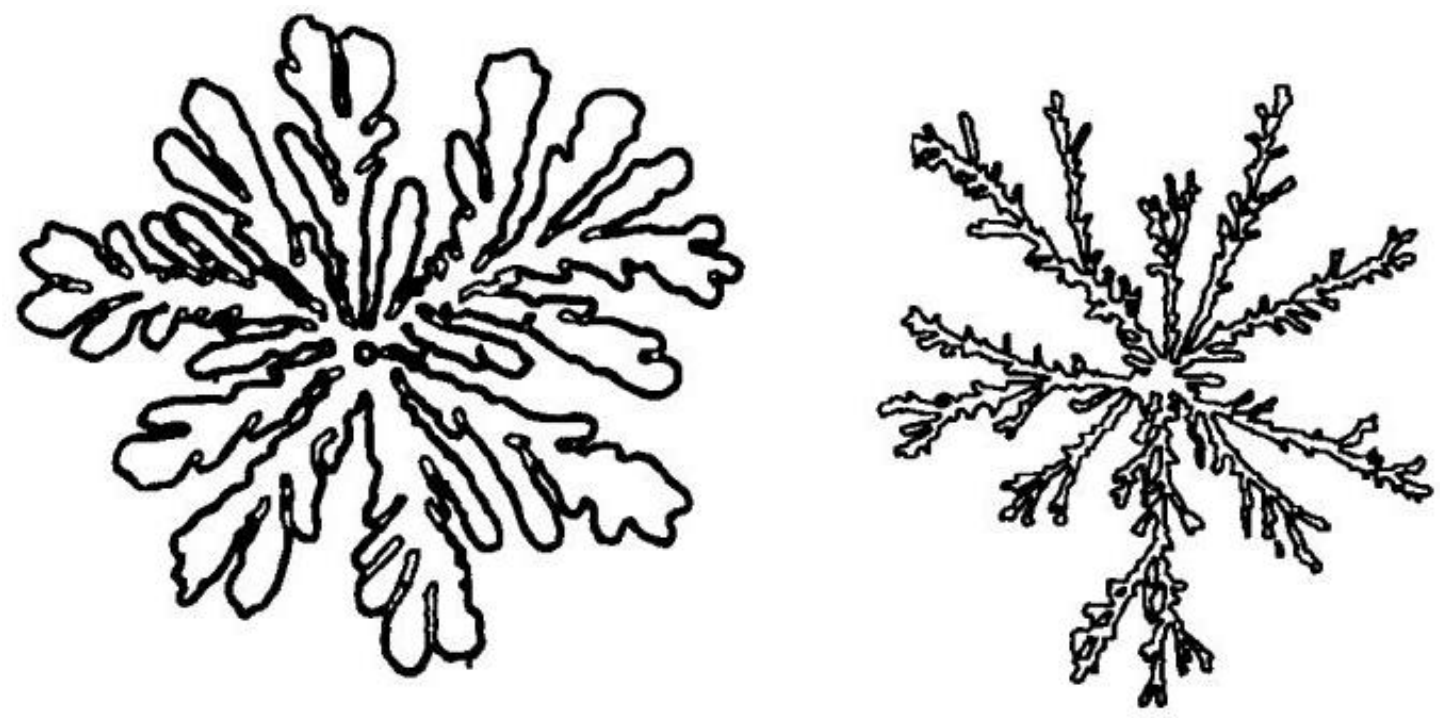

Figure 2.5 - Injection of a low viscosity fluid in a high viscosity fluid. (a) Air displacing glycerol. (b) Water displacing a non-Newtonian high viscosity mixture of sclerogutan in water (Feder 1988). This phenomenon is known as viscous fingering and it has been shown experimentally to possess fractal properties (Maloy et al. 1987).

On the other hand, an in-depth discussion of fractal theory may involve rather abstract mathematical concepts such as Hausdorff dimensions, algebraic topology, multivariate statistics (Feder 1988), etc. While intellectually stimulating, mastery of these concepts adds little practical value to the reservoir 
engineer's arsenal. The practicing engineer, who is in need of fast and practical solutions, is probably not inclined to spend the time and effort required to master these theoretical concepts simply out of intellectual curiosity. Furthermore, it is difficult, if not impossible, to determine a priori the fractal dimensions of a reservoir, and no work has been published that proposes a workflow with fractals as an integral part of the reservoir characterization process. It is our impression, based on an exhaustive literature review, that the application of fractals to reservoir engineering is considered a niche specialization that is mostly used as a last-ditch effort when the more traditional methods fail to characterize naturally-fractured reservoirs.

The work of Chang and Yortsos (1990) contains the basic theoretical formalism as it pertains to petroleum engineering applications. Their contribution consisted of a modification of the Warren-Root model so that instead of having a network of linearly arranged matrix "sugar cubes," the permeable fractures embedded within the matrix would be arranged in a fractal fashion (Figure 2.6). This is a powerful approach because it made possible the development of a new, 'fractal' diffusivity equation, which in turn allowed for pressure-transient testing of naturally-fractured reservoirs that may exhibit a fractal fracture distribution. This work was further advanced and numerically tested in 2-D networks of fractures by Acuña and Yortsos (1991). These authors proposed a flexible numerical method for the construction of fractal networks of a variety of geometrical and connectivity properties. 


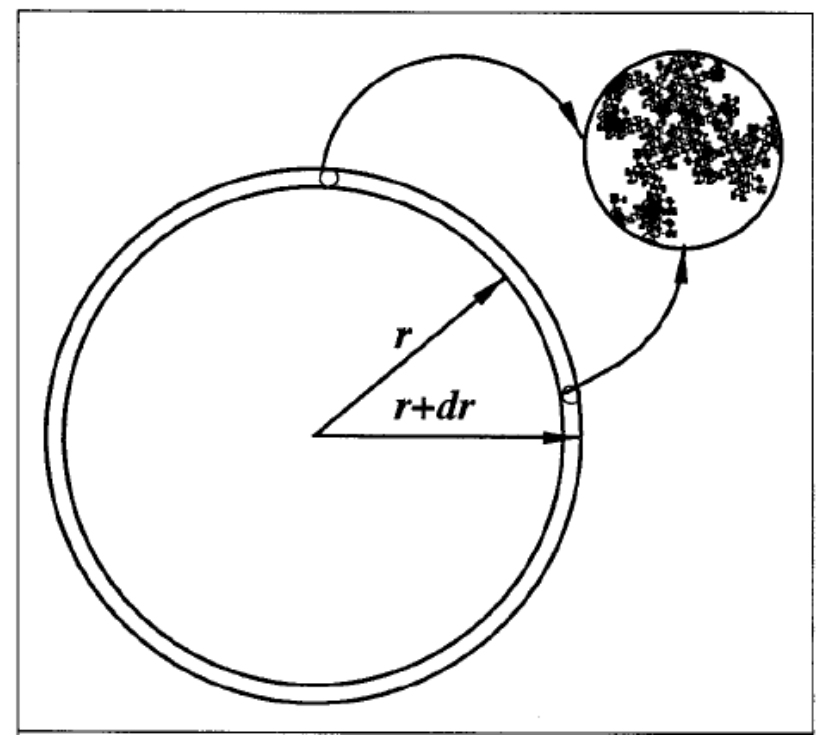

Figure 2.6 - Schematic of a fractal fracture network embedded in a Euclidean matrix (Chang and Yortsos 1990)

Flamenco-López and Camacho-Velázquez (2003) investigated the transient flow regime and showed that it is possible, by combining the transient and pseudosteady-state responses, to obtain via history-matching values for all four parameters of their fractal model. Beier (1994) extended the fractal model of Chang and Yortsos (1990) to consider a hydraulically fractured well. He also observed a power-law behavior during the linear and radial flow periods. Camacho-Velázquez et al (2008) applied the fractal model to generate a series of decline curves, for which they successfully applied to well performance data obtained from a naturally-fractured reservoir field case.

Lastly, Fuentes-Cruz et al. (2010) used the fractal concept to propose a unified approach for fall-off/buildup tests with short injection/production times. At the core of their study is a "'radial composite model with a fractal transition zone," which essentially argues that there is a fractal viscous fingering phenomenon occurring in the transition zone created by the injection fluid, as shown in Figure 2.7. Finally, Hardy and Beier (1994) prepared a comprehensive review of fractals applied to geology and reservoir engineering, and we would comment that their focus is toward statistical aspects of the problem. 


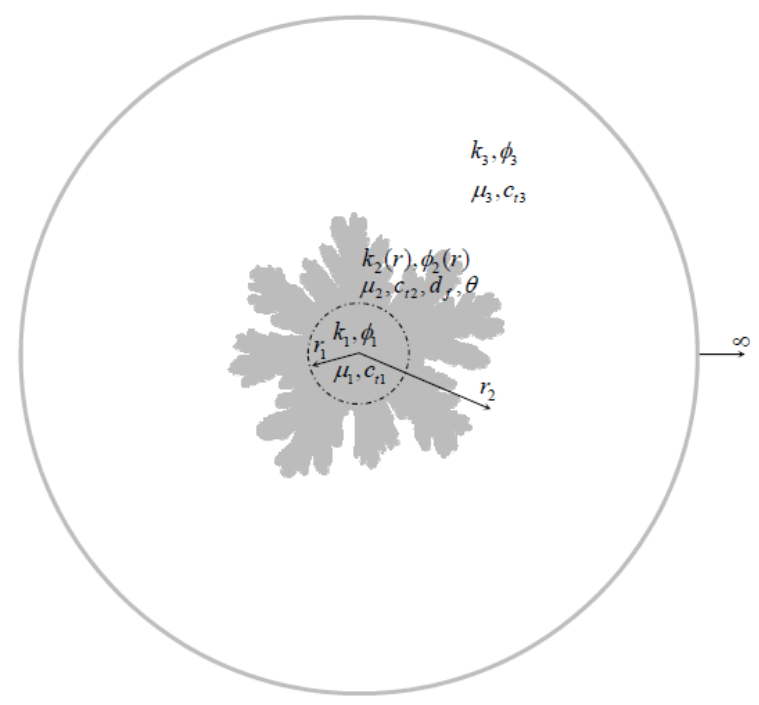

Figure 2.7 - Radial Composite Model with Fractal Transition Zone (Fuentes-Cruz et al. 2010) 


\subsection{Reservoir Engineering Problems Related to Tight Gas/Shale Gas Reservoirs}

The problem of understanding flow in a hydraulic fracture has been addressed extensively in the literature; a comprehensive review may be found in Blasingame and Poe (1993). It has gained increased importance in recent years because of the surge in unconventional gas exploitation. However, due to the multitude of possible unknowns that can influence the conditions of the problem, different solutions must be developed for each case (Ozkan and Raghavan 1991).

A hydraulic fracture is usually vertical, and it may be derived from a single treatment (e.g., a vertical well) or part of a larger stimulation treatment (e.g., a multi-stage hydraulic fracture treatment in a horizontal well). In the case of shale and coal-bed methane reservoirs, the effects of desorption may need to be taken into account, as well as non-Darcy flow. Furthermore, a low gas price and/or high oil price may encourage operators to look for tight gas condensate and volatile oil reservoirs (Dar 2010), where this effort introduces a non-trivial multiphase flow consideration to the problem. Geomechanical effects may also play an important role in the production life (Nagel et al. 2012). Finally, the reservoir may also be naturally-fractured. All of these parameters collude to make the problem especially challenging and almost certainly beyond the capability of classic analytical solutions.

We have mentioned a host of parameters which can have a major impact on the production performance of a hydraulically fractured well - however; at present, we have only considered the parameters that depend on the geology and in-situ reservoir conditions. Another full layer of complexity is added when one considers the unknowns which depend on the operational decisions controlling production. The fracture may only partially penetrate the pay zone. Conversely, if the fractures achieve a great vertical height, production from several commingled layers is possible.

The exact dimensions and characteristics of created hydraulic fractures are elusive, as micro-seismic techniques can give an idea of where the micro-seismic events take place, but this does not necessarily mean that the fracture has been sufficiently opened (propped) to allow measurable production from these locations (Baihly et al. 2006). Some fractures may suffer from formation damage from excess water 
presence near their faces. Finally, there is a host of proppant choices, and incorrect proppant selection or ineffective transport of the proppant through the fracture may hinder the hydraulic conductivity of the fracture.

We have mentioned several parameters and conditions that can complicate the physical model of tight and shale gas reservoirs. These problems may be tackled satisfactorily using numerical simulation. However, comprehensive reservoir simulation is expensive in terms of manpower, computational power, time and budget. Therefore, researchers are encouraged to develop analytical or semi-analytical solutions that can capture at least some of these most important physical complexities of state-of-the-art problems so that design, analysis, and interpretation tasks can be performed.

Beier (1994) applied instantaneous line-source functions, in the same spirit as Gringarten et al (1974), to a single vertical fracture fully penetrating a reservoir that is assumed to have a permeable fracture network that is fractally distributed. He considered the cases of infinite-conductivity and uniform flux, and successfully applied it to a field case. As this is the only publication that applies fractal theory specifically to hydraulic fractures which we are aware of, we believe that this is a research area that has largely been unexplored, and the remainder of this thesis is devoted to this particular problem. 


\section{A STUDY OF THE FRACTAL DIFFUSIVITY EQUATION}

\subsection{Derivation of the Fractal Diffusivity Equation}

The Fractal Diffusivity Equation forms the backbone of this thesis. It is important that its detailed derivation, from basic principles to its final dimensionless form, be fully documented. In this sub-section we derive a $2 \mathrm{D}$ result for anisotropic media and state some possible 2D applications and solution methods. Subsequently we reduce the formulation to a 1D form and finish by deriving its dimensionless form where all variable are in the SI unit system.

In this thesis we use the power-law based formalism introduced by Chang and Yortsos (1990) that describes the porosity and permeability of a reservoir as a function of distance from the wellbore. The simplified relations that we choose to work with are

$$
\begin{aligned}
& k(x)=k_{w}\left(\frac{x}{x_{w}}\right)^{d-\theta-2} \\
& \phi(x)=\phi_{w}\left(\frac{x}{x_{w}}\right)^{d-2}
\end{aligned}
$$

Moving to the next sequence of relations, we make the following definitions:

- $k_{w}$ is the permeability at the edge of the wellbore $\left(\mathrm{m}^{2}\right)$

- $\phi_{w}$ is porosity at the edge of the wellbore (fraction)

- $x_{w}$ is the distance from the center of wellbore to the edge of the wellbore $(\mathrm{m})$

- $d$ is the fractal dimension of the pore spaces (dimensionless)

- $\theta$ is the fractal dimension of the fluid flow (dimensionless)

- The subscripts $x$ and $y$ indicate the $x$ and $y$ directions, respectively

These relations are valid for 1D systems. Originally these relations were introduced to model the transient response of naturally-fractured reservoirs whose fracture network could be assumed to have a fractal distribution. The first "fractal" diffusivity equation (in radial coordinates) was proposed by Chang and 
Yortsos (1990). To our knowledge, these types of fractal relations have only been used to study the heterogeneity of a reservoir.

Yun et al. (2009) and Kong et al. (2009) extended Eqs. 3.1 and 3.2 to 2D and 3D Cartesian coordinate systems (respectively) and proceeded to derive their own fractal diffusivity equations. In this thesis we restrict our analysis to $2 \mathrm{D}$ and use the following definitions:

$$
\begin{aligned}
& k_{x}(x)=k_{w x}\left(\frac{x}{x_{w}}\right)^{d_{x}-\theta_{x}-2} . \\
& k_{y}(y)=k_{w y}\left(\frac{y}{y_{w}}\right)^{d_{y}-\theta_{y}-2} .
\end{aligned}
$$

Where Eqs. 3.3 and 3.4 are used for the permeabilities in $2 \mathrm{D}-$ similarly, the porosity in $2 \mathrm{D}$ is defined (Kong et al. 2009) as

$$
\phi(x, y)=\frac{\phi_{w}}{2}\left[\left(\frac{x}{x_{w}}\right)^{d_{x}-2}+\left(\frac{y}{y_{w}}\right)^{d_{y}-2}\right]
$$

It is important to note that the "fractal" nomenclature changes from author to author and there is no "standard" approach; this is why we provide all definitions and derivations in this thesis. We begin with the Continuity Equation (Lee and Wattenbarger 1996) for a slightly compressible, single-phase flow in a porous medium

$$
-\nabla \cdot(\rho \vec{v})=\frac{\partial}{\partial t}(\phi \rho)
$$

Where:

$\rho$ is the fluid density $\left(\mathrm{kg}-\mathrm{m}^{-3}\right)$,

$\phi$ is the porosity (fraction),

$v$ is the velocity $\left(\mathrm{m}-\mathrm{s}^{-1}\right)$, and

$t$ is time in (s). 
To model permeability in a $2 \mathrm{D}$ anisotropic media, a permeability tensor is needed. Pressure can be applied in two directions, and for each direction, permeability can be measured (via Darcy's law in 2D) in two directions - thus leading to a 2 by 2 tensor (Bear 1972). In this case we use the permeability tensor of the form $\left[\begin{array}{cc}k_{x} & 0 \\ 0 & k_{y}\end{array}\right]$. Darcy's Law for an anisotropic, orthotropic medium in a 2D Cartesian coordinate system (Ferrandon 1948) may be expressed as

$$
\vec{v}=\left\{-v_{x} ;-v_{y}\right\}=\left\{-\frac{1}{\mu} k_{x} \frac{\partial p}{\partial x} ;-\frac{1}{\mu} k_{y} \frac{\partial p}{\partial y}\right\}
$$

Where:

$$
\begin{aligned}
& \mu \text { is the viscosity in (Pa-s), } \\
& p \text { is the pressure (Pa), and } \\
& k \text { is the permeability }\left(\mathrm{m}^{2}\right) .
\end{aligned}
$$

As we only consider 2-D horizontal flow in the $(x, y)$ plane, then we can ignore gravity effects in the $z$ direction. For the case of a scalar $\psi$ and a vector $\vec{A}$, then the following vector calculus identity exists: (Kreyszig 2005)

$$
\nabla \cdot(\psi \vec{A})=\psi(\nabla \cdot \vec{A})+\vec{A} \cdot(\nabla \psi)
$$

Noting that $\rho$ is a scalar and $\vec{v}$ a vector, the Left-Hand-Side (LHS) of Eq. 3.6 becomes:

$$
\begin{aligned}
-\nabla \cdot(\rho \vec{v}) & =-[\rho(\nabla \cdot \vec{v})+\vec{v} \cdot(\nabla \rho)] \\
& =\rho\left[\frac{\partial v_{x}}{\partial x}+\frac{\partial v_{y}}{\partial y}\right]+\vec{v} \cdot \nabla \rho \\
& =\rho\left[\frac{\partial v_{x}}{\partial x}+\frac{\partial v_{y}}{\partial y}\right]+v_{x} \frac{\partial \rho}{\partial x}+v_{y} \frac{\partial \rho}{\partial y} \\
& =\frac{1}{\mu}\left[\rho\left\{\frac{\partial}{\partial x}\left[k_{x} \frac{\partial p}{\partial x}\right]+\frac{\partial}{\partial y}\left[k_{y} \frac{\partial p}{\partial y}\right]\right\}+k_{x} \frac{\partial p}{\partial x} \frac{\partial \rho}{\partial x}+k_{y} \frac{\partial p}{\partial y} \frac{\partial \rho}{\partial y}\right] .
\end{aligned}
$$

Application of the chain rule yields: 


$$
\frac{\partial \rho}{\partial x} \frac{\partial p}{\partial x}=\frac{\partial \rho}{\partial p} \frac{\partial p}{\partial x} \frac{\partial p}{\partial x}=\frac{\partial \rho}{\partial p}\left(\frac{\partial p}{\partial x}\right)^{2}
$$

Where we assume this term can be ignored because this term is typically very small. The same is true for the $\frac{\partial \rho}{\partial y} \frac{\partial p}{\partial y}$ term. The LHS can further be expanded to yield:

$$
L H S=\frac{\rho}{\mu}\left[k_{x} \frac{\partial^{2} p}{\partial x^{2}}+k_{y} \frac{\partial^{2} p}{\partial y^{2}}+\frac{\partial k_{x}}{\partial x} \frac{\partial p}{\partial x}+\frac{\partial k_{y}}{\partial y} \frac{\partial p}{\partial y}\right]
$$

Introducing the anisotropic fractal relations of permeability, Eqs. 3.3 and 3.4, into the LHS of Eq. 3.10 yields:

$$
\begin{aligned}
L H S=\frac{\rho}{\mu}[ & k_{w x}\left(\frac{x}{x_{w}}\right)^{d_{x}-\theta_{x}-2} \frac{\partial^{2} p}{\partial x^{2}}+k_{w y}\left(\frac{y}{y_{w}}\right)^{d_{y}-\theta_{y}-2} \frac{\partial^{2} p}{\partial y^{2}} \\
& \left.+k_{w x}\left(\frac{x}{x_{w}}\right)^{d_{x}-\theta_{x}-2} \frac{d_{x}-\theta_{x}-2}{x} \frac{\partial p}{\partial x}+k_{w y}\left(\frac{y}{y_{w}}\right)^{d_{y}-\theta_{y}-2} \frac{d_{y}-\theta_{y}-2}{y} \frac{\partial p}{\partial y}\right]
\end{aligned}
$$

For mathematical convenience, we define the wellbore distances $x_{w}$ and $y_{w}$ to be equal. Similarly, we define the permeabilities at the wellbore $k_{w x}$ and $k_{w y}$ to be equal. We force equivalency at the wellbores only, we are not making the system isotropic. The system will still be anisotropic if $d_{x} \neq d_{y}$ and/or $\theta_{x} \neq \theta_{y}$ since the permeabilities and porosities can still be different over the length of the two axes $x$ and $y$. After setting $k_{w x}=k_{w y}=k_{w}$ and $x_{w}=y_{w}=\lambda$, Eq. 3.11 becomes:

$$
\begin{aligned}
L H S= & \frac{\rho k_{w}}{\mu \lambda^{d-\theta-2}}\left[x^{d-\theta-2} \frac{\partial^{2} p}{\partial x^{2}}+y^{d-\theta-2} \frac{\partial^{2} p}{\partial y^{2}}+x^{d-\theta-2} \frac{d-\theta-2}{x} \frac{\partial p}{\partial x}\right. \\
& \left.+y^{d-\theta-2} \frac{d-\theta-2}{y} \frac{\partial p}{\partial y}\right]
\end{aligned}
$$


Expansion of the Right-Hand-Side (RHS) of the continuity equation (Eq. 3.6) yields:

$$
\text { RHS }=\frac{\partial}{\partial t}(\phi \rho)=\frac{\partial(\phi \rho)}{\partial p} \frac{\partial p}{\partial t}=\left[\phi \frac{\partial \rho}{\partial p}+\rho \frac{\partial \phi}{\partial p}\right] \frac{\partial p}{\partial t}=\phi \rho\left[\frac{1}{\rho} \frac{\partial \rho}{\partial p}+\frac{1}{\phi} \frac{\partial \phi}{\partial p}\right] \frac{\partial p}{\partial t} \ldots
$$

By definition:

$$
\begin{aligned}
& c_{f}=\frac{1}{\rho} \frac{\partial \rho}{\partial p} \quad \text { (fluid compressibility) } \\
& c_{r}=\frac{1}{\phi} \frac{\partial \phi}{\partial p} \text { (rock compressibility) }
\end{aligned}
$$

Where both $c_{f}$ and $c_{r}$ are in units of $\mathrm{Pa}^{-1}$. Therefore, the total compressibility is expressed as $c_{t}=c_{f}+c_{r}$, and substitution into Eq. 3.13 results in the simple relatively simple form:

$$
R H S=\phi \rho c_{t} \frac{\partial p}{\partial t}
$$

Inserting the 2D anisotropic fractal definition of porosity (Eq. 3.5) into the RHS leads to

$$
R H S=\rho c_{t} \frac{\phi_{w}}{2}\left[\left(\frac{x}{x_{w}}\right)^{d-2}+\left(\frac{y}{y_{w}}\right)^{d-2}\right] \frac{\partial p}{\partial t}
$$

Similar to Eq. 3.12, we set $x_{w}=y_{w}=\lambda$. The final form of the RHS becomes

$$
R H S=\frac{\rho c_{t} \phi_{w}}{2 \lambda^{d-2}}\left[x^{d-2}+y^{d-2}\right] \frac{\partial p}{\partial t}
$$

Equating the LHS and RHS using Eq. 3.12 and Eq. 3.15, we obtain:

$$
\begin{aligned}
& \frac{\rho k_{w}}{\mu \lambda^{d-\theta-2}}\left[x^{d-\theta-2} \frac{\partial^{2} p}{\partial x^{2}}+y^{d-\theta-2} \frac{\partial^{2} p}{\partial y^{2}}+x^{d-\theta-2} \frac{d-\theta-2}{x} \frac{\partial p}{\partial x}+y^{d-\theta-2} \frac{d-\theta-2}{y} \frac{\partial p}{\partial y}\right] \\
& =\frac{\rho c_{t} \phi_{w}}{2 \lambda^{d-2}}\left[x^{d-2}+y^{d-2}\right] \frac{\partial p}{\partial t}
\end{aligned}
$$

Which, after some manipulation yields the following relation: 


$$
\begin{aligned}
& x^{d-\theta-2} \frac{\partial^{2} p}{\partial x^{2}}+y^{d-\theta-2} \frac{\partial^{2} p}{\partial y^{2}}+x^{d-\theta-2} \frac{d-\theta-2}{x} \frac{\partial p}{\partial x} \\
& +y^{d-\theta-2} \frac{d-\theta-2}{y} \frac{\partial p}{\partial y}=\frac{\mu c_{T t}}{2 \lambda^{\theta}} \frac{\phi_{w}}{k_{w}}\left[x^{d-2}+y^{d-2}\right] \frac{\partial p}{\partial t}
\end{aligned}
$$

Equation 3.16 is the anisotropic form of the 2D fractal diffusivity equation. Kong et al. (2009) present an analogous form to Eq. 3.16, but they begin from a different expression for porosity and permeability and do not provide the intermediate steps. Possible applications of a 2D anisotropic diffusivity equation such as Eq. 3.16 include the study of directional permeability in coal seams (Wold and Jeffrey 1999), optimizing placement of horizontal wells (Muñoz et al. 1998), and the study of $\mathrm{CO}_{2}$ dissolution in deep saline aquifers (Taheri et al. 2012). Such an expression may be solved using Fourier transforms (Carslaw and Jaeger 1959), coordinate system transformations (Sheng 2010) or via numerical methods (Friedrich and Gurevich 2010).

We now reduce Eq. 3.16 to its $1 \mathrm{D}$ form by forcing $y=0$ at all times. In addition, Eq. 3.2 is combined with Eq. 3.14a to yield:

$$
x^{d-\theta-2} \frac{\partial^{2} p}{\partial x^{2}}+x^{d-\theta-2} \frac{d-\theta-2}{x} \frac{\partial p}{\partial x}=\frac{\mu c_{T}}{x_{w} \theta} \frac{\phi_{w}}{k_{w}}\left[x^{d-2}\right] \frac{\partial p}{\partial t}
$$

or

$$
\frac{\partial^{2} p}{\partial x^{2}}+\frac{d-\theta-2}{x} \frac{\partial p}{\partial x}=\mu c_{T} \frac{\phi_{w}}{k_{w}}\left(\frac{x}{x_{w}}\right)^{\theta} \frac{\partial p}{\partial t}
$$

Equation 3.17 is analogous, but not identical, to the 1D Cartesian fractal diffusivity equation developed by Kong et al (2009) and Yun et al (2009). Using a very similar approach it is possible to derive a radial fractal diffusivity equation, just as Chang and Yortsos (1990). While Eqs. 3.16 and 3.17 are not new, we have verified that these relations are correct by comparing with 3 different sources. We also provide a detailed derivation of these relations. 
By setting $d=2$ and $\theta=0$, the classic Euclidean $1 \mathrm{D}$ linear diffusivity equation is recovered. This corresponds to a constant permeability and porosity throughout the reservoir.

$$
\frac{\partial^{2} p}{\partial x^{2}}=\mu c_{t} \frac{\phi_{w}}{k_{w}} \frac{\partial p}{\partial t}
$$

We also develop the dimensionless version of Eq. 3.17 by following the traditional procedure for the Euclidean linear diffusivity equation (Blasingame 2010a). The dimensionless spatial variable is defined as

$$
x_{D}=\frac{x}{x_{w}}
$$

Substitution of Eq. 3.19 into Eq. 3.17 yields,

$$
\frac{d^{2} p}{d\left(x_{D} x_{w}\right)^{2}}+\frac{d-\theta-2}{x_{D} x_{w}} \frac{d p}{d\left(x_{D} x_{w}\right)}=\mu c_{t} \frac{\phi_{w}}{k_{w}}\left(\frac{x}{x_{w}}\right)^{\theta} \frac{d p}{d t}
$$

which, after a rearrangement of terms, results in

$$
\frac{d^{2} p}{d x_{D}^{2}}+\frac{d-\theta-2}{x_{D}} \frac{d p}{d x_{D}}=\mu c_{t} \frac{\phi_{w}}{k_{w}} x_{w}^{2} x_{D}^{\theta} \frac{d p}{d t}
$$

The dimensionless pressure $p_{D}$ is defined as

$$
p_{D}=\frac{1}{p_{c h}}\left(p_{i}-p\right),
$$

Where:

$p_{i}$ is the initial reservoir pressure $(\mathrm{Pa})$, and

$p_{c h}$ is the characteristic pressure $(\mathrm{Pa})$.

The appropriate value of the characteristic pressure will be defined using the inner boundary condition; and from Darcy's law, the inner boundary condition for a constant rate case is given as:

$$
q=\frac{k_{w} A}{B \mu}\left[\frac{d p}{d x}\right]_{x=x_{w}}
$$


Where Eq. 3.23 can be rewritten as:

$$
\left[\frac{d p}{d x}\right]_{x=x_{w}}=\frac{q B \mu}{k_{w} A}
$$

Substituting in Eqs. 3.19 and 3.22 into Eq. 3.24 yields:

$$
\left[\frac{d\left(p_{i}-p_{D} p_{c h}\right)}{d\left(x_{D} x_{w}\right)}\right]_{x_{D}=1}=\frac{q B \mu}{k_{w} A}
$$

Recalling that $p_{i}, x_{w}$ and $p_{c h}$ are constants, Eq. 3.25 becomes:

$$
\left[\frac{d p_{D}}{d x_{D}}\right]_{x_{D}=1}=-\frac{1}{p_{c h}} \frac{q B \mu x_{w}}{k_{w} A}
$$

For mathematical convenience, the following dimensionless inner boundary condition is imposed:

$$
\left[\frac{d p_{D}}{d x_{D}}\right]_{x_{D}=1}=-1
$$

For Eq. 3.27 to be true, then according to Eq. 3.26 the following definition must be true

$$
p_{c h}=\frac{q B \mu x_{w}}{k_{w} A}
$$

Where the final form of the dimensionless pressure is given as:

$$
p_{D}=\frac{k_{w} A}{q B \mu x_{w}}\left(p_{i}-p\right)
$$

The dimensionless time variable is defined using "what is leftover" on the Right-Hand-Side (RHS). Solving Eq. 3.22 for $p$ and substituting this result into Eq. 3.21 leads to

$$
\frac{d^{2}\left(p_{i}-p_{D} p_{c h}\right)}{d x_{D}{ }^{2}}+\frac{d-\theta-2}{x_{D}} \frac{d\left(p_{i}-p_{D} p_{c h}\right)}{d x_{D}}=\mu c_{t} \frac{\phi_{w}}{k_{w}} x_{w}{ }^{2} x_{D} \theta \frac{d\left(p_{i}-p_{D} p_{c h}\right)}{d t}
$$

Recall that the initial and characteristic pressures are both constants, then their derivative terms are eliminated, which results in the following form: 


$$
\frac{d^{2} p_{D}}{d x_{D}{ }^{2}}+\frac{d-\theta-2}{x_{D}} \frac{d p_{D}}{d x_{D}}=\mu c_{t} \frac{\phi_{w}}{k_{w}} x_{w}^{2} x_{D} \theta \frac{d p_{D}}{d t}
$$

From the RHS of Eq. 3.31, the dimensionless time variable is defined as:

$$
t_{D}=\frac{k_{w}}{\phi_{w} \mu c_{T} x_{w}^{2}} t
$$

The final form of the Fractal Diffusivity Equation is thus

$$
\frac{\partial^{2} p_{D}}{\partial x_{D}^{2}}+\frac{d-\theta-2}{x_{D}} \frac{\partial p_{D}}{\partial x_{D}}=x_{D} \theta \frac{\partial p_{D}}{\partial t_{D}}
$$

Where the dimensionless space variable $x_{D}$, the dimensionless pressure $p_{D}$, and the dimensionless time $t_{D}$ are defined by Eqs. 3.19, 3.29, and 3.32 respectively.

\subsection{Discussion of the Fractal Porosity Permeability Relations}

Now that we have derived the $1 \mathrm{D}$ dimensionless fractal diffusivity equation, we will work only with this expression for the rest of the thesis. Having said this, there are a couple of important observations to be made about Eqs. 3.1 and 3.2, to which we will hereafter refer to as Fractal Porosity-Permeability Relations (FPPR):

- A cursory numerical exercise using typical conventional reservoir values reveals that, if $d \neq 2$ (and/or $\theta \neq 0$ ), then the FPPR describes a situation that may be physically improbable — or even impossible. For example, if we set $x_{w}=0.10 \mathrm{~m}, \phi_{w}=15 \%$ (or 0.15 fraction), $k_{w}=1.9738 \times 10^{-13} \mathrm{~m}^{2}(200 \mathrm{md}$ ), $d=$ 2.5 and $\theta=0$, we find that at 20 meters from the wellbore, we should expect a permeability of 2.82 Darcy and a porosity of $212.13 \%$ !

- While the physical meaning of parameters $d$ and $\theta$ is generally well understood by specialists, they remain conceptually elusive, as these parameters cannot be easily related to tangible physical quantities. This makes these concepts difficult to digest by non-specialized practitioners, hindering their widespread acceptance. 
- When discussing the physical meaning of these relations, Acuña et al (1995) are careful to issue a caveat, stating that "it must be stressed again that they do not correspond to point values (local averages) but to the porosity and permeability of regions of size $r$. It should be also stressed that [Eqs. 3.1 and 3.2] do not imply that the conventional porosity and permeability are radially dependent around a given well. They only suggest that in a fractal medium, all properties of any region of size r are scale-dependent following a power law".

For these reasons, the FPPR have not received much attention in the literature, and to our knowledge, no attempt has been made up until now to produce new solutions or methodologies based on the idea of a radially changing porosity and permeability in the literal sense of the definition.

In spite of all of these apparent drawbacks, the FPPR do possess interesting features that make their additional study worth our efforts. A classical analysis of fluid flow in porous media with a constant permeability and porosity throughout the reservoir results in the following 1D diffusivity equations

$$
\begin{aligned}
& \frac{\partial^{2} p_{D}}{\partial x_{D}{ }^{2}}=\frac{\partial p_{D}}{\partial t_{D}} . \\
& \frac{\partial^{2} p_{D}}{\partial r_{D}{ }^{2}}+\frac{1}{r_{D}} \frac{\partial p_{D}}{\partial r_{D}}=\frac{\partial p_{D}}{\partial t_{D}}
\end{aligned}
$$

Where:

$p_{D}$ is the dimensionless pressure, $r_{D}$ is the dimensionless wellbore radius in radial coordinates, and $x_{D}$ is the dimensionless wellbore radius in Cartesian coordinates.

Eqs. 3.34 and 3.35 are expressed in dimensionless variables and are derived, starting from the continuity equation, in Cartesian and radial coordinate systems, respectively. A cursory inspection of the Fractal Diffusivity Equation (Eq. 3.33) yields the following interesting observations:

- If we set $\{d=2, \theta=0\}$, we obtain exactly Eq. 3.34 from Eq. 3.33. This means we are dealing with a linear reservoir with constant hydraulic properties, i.e. the classic Euclidean linear flow case. 
- Similarly, if we select $\{d=3, \theta=0\}$, we obtain exactly Eq. 3.35 from Eq. 3.33. Recalling that Eq. 3.33 was derived in Cartesian coordinates and Eq. 3.35 in radial coordinates, this implies that a constanthydraulic-properties radial flow is equivalent to a linearly-increasing-hydraulic-properties linear flow. As this is an important concept, we provide an illustration in Figure 3.1.

This seems to suggest that, at least mathematically, the FDE (Eq. 3.33) is more fundamental than Eqs. 3.34 or 3.35, and can act as a sort of bridge between the two classic flow regimes, linear and radial. It also begs the question as to what happens when we choose a value for $d$ that is neither 2 nor 3 . Are we describing something that is 'in between' the two regimes (such as elliptical flow)? Expanding this idea, we can contemplate a situation where we find solutions to non-trivial flow regimes - e.g., involving multiple fractures, by exploiting the flexibility of the FDE.

\subsection{Analytical Study}

We provide the steps to solve Eq. 3.33 in the Laplace domain for a no-flow outer boundary condition. This equation has the following initial and boundary conditions:

Initial condition: $\quad p_{D}=0$ when $t_{D}=0$

Inner boundary condition:

$\left.\left[\frac{d p_{D}}{d x_{D}}\right]\right|_{x_{D}=1}=-1$

No-flow, outer boundary condition: $\left.\quad\left[\frac{d p_{D}}{d x_{D}}\right]\right|_{x_{D}=L}=0$

where $L$ is the dimensionless distance to the no-flow outer boundary. 
$\frac{\partial^{2} p_{D}}{\partial r_{D}^{2}}+\frac{1}{r_{D}} \frac{\partial p_{D}}{\partial r_{D}}=\frac{\partial p_{D}}{\partial t_{D}}$

Classic radial diffusivity equation

A radial system with constant $\{k, \phi\}$ properties

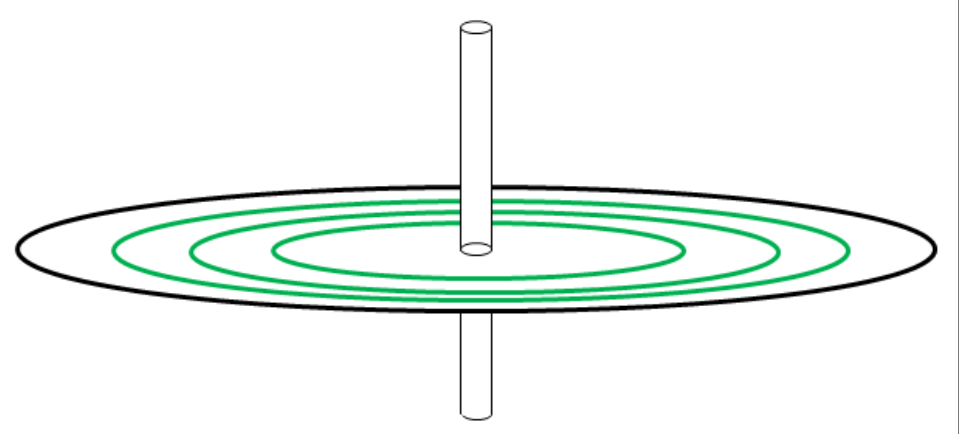

is equivalent to

$\frac{\partial^{2} p_{D}}{\partial x_{D}^{2}}+\frac{d-\theta-2}{x_{D}} \frac{\partial p_{D}}{\partial x_{D}}=x_{D}^{\theta} \frac{\partial p_{D}}{\partial t_{D}}$

Fractal diffusivity equation with $\{d=3, \theta=0\}$

A linear system with linearly increasing $\{k, \phi\}$ properties

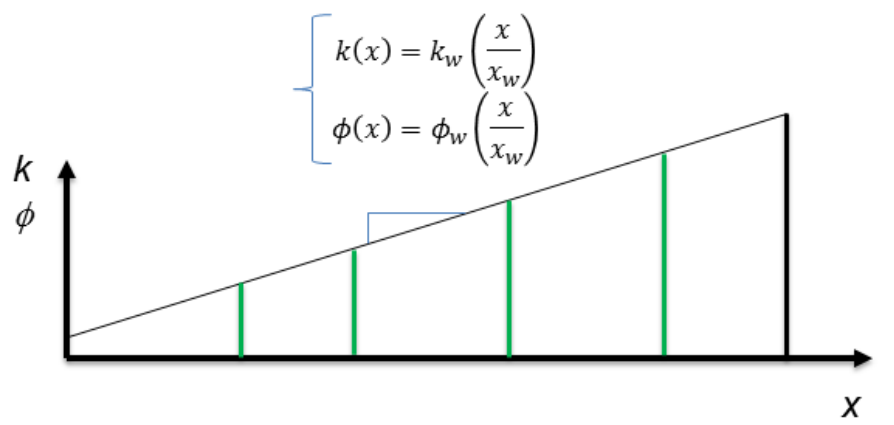

Figure 3.1 - Schematic of the equivalency between fractal linear flow and classic radial flow. Even though the top and bottom cases describe different physical scenarios, the Fractal Diffusivity Equation predicts that both should yield the same pressure signal at the wellbore. This has been verified analytically and numerically in this thesis.

Taking the Laplace transform of Eq. 3.33, and using Eq. 3.36a, results in

$$
\frac{d^{2} \overline{p_{D}}}{d x_{D}{ }^{2}}+\frac{d-\theta-2}{x_{D}} \frac{d \overline{p_{D}}}{d x_{D}}=x_{D}^{\theta} z \overline{p_{D}}
$$

Upon initial inspection, it is not immediately obvious what the general solution to this equation may be as this form does not fit any of the classic Bessel ordinary differential equations (standard or modified). As a reference, Bowman (1958) presents a very general form of the modified Bessel differential equation as

$$
\frac{d^{2} y}{d x^{2}}+\frac{1-2 \alpha}{x} \frac{d y}{d x}=\left(\beta^{2} \gamma^{2} x^{2 \gamma-2}+\frac{\alpha^{2}-n^{2} \gamma^{2}}{x^{2}}\right) y,
$$


Where $y=y(x)$ and $\alpha, \beta, \gamma$ and $n$ are real constants that must be determined (Bowman 1958); Eq. 3.38 has the general solution given by:

$$
y(x)=x^{\alpha}\left(A I_{n}\left[\beta x^{\gamma}\right]+B K_{n}\left[\beta x^{\gamma}\right]\right)
$$

Where the functions $I_{n}$ and $K_{n}$ are modified Bessel functions. In order to equate the previous relations with the form given by Eq. 3.39, we must reformulate these into the $\alpha, \beta, \gamma$ and $n$ parameters. Each of these equalities are provided below:

Determine $\alpha$ :

$$
\begin{gathered}
1-2 \alpha=d-\theta-2 \\
2 \alpha=-d+\theta+3 \\
\alpha=\frac{\theta+3-d}{2}
\end{gathered}
$$

Determine $\gamma$ :

$$
\begin{aligned}
& 2 \gamma-2=\theta \\
& \gamma=\frac{\theta+2}{2}
\end{aligned}
$$

Determine $n$ :

$$
\begin{gathered}
\alpha^{2}-n^{2} \gamma^{2}=0 \\
\left(\frac{\theta+3-d}{2}\right)^{2}=n^{2}\left(\frac{\theta+2}{2}\right)^{2} \\
n=\frac{\theta+3-d}{\theta+2}
\end{gathered}
$$

Determine $\beta$ :

$$
\begin{gathered}
\beta^{2} \gamma^{2}=(z+\sqrt{z}) \\
\beta=\frac{\sqrt{z}}{\gamma}
\end{gathered}
$$


Therefore, the general solution to Eq. 3.37 can be written as:

$$
\overline{p_{D}}\left(x_{D}, z\right)=x_{D}^{\alpha}\left\{A I_{n}\left[\frac{\sqrt{z}}{\gamma} x_{D}^{\gamma}\right]+B K_{n}\left[\frac{\sqrt{z}}{\gamma} x_{D}^{\gamma}\right]\right\}
$$

Both boundary conditions (Eqs $3.36 \mathrm{~b}$ and $3.36 \mathrm{c}$ ) necessitate computation of the derivative $\frac{d \overline{P_{D}}}{d x_{D}}$. This is not a trivial computation, but can be accomplished by using the mathematical manipulations discussed below. From the properties of the derivatives of modified Bessel functions (Bowman 1958):

$$
\begin{aligned}
& I_{n}^{\prime}[f(x)]=\left(I_{n-1}[f(x)]-\frac{n}{f(x)} I_{n}[f(x)]\right) f^{\prime}(x) \ldots \ldots . . \\
& K_{n}^{\prime}[f(x)]=-\left(K_{n-1}[f(x)]+\frac{n}{f(x)} K_{n}[f(x)]\right) f^{\prime}(x) .
\end{aligned}
$$

Using the product rule

$$
\begin{aligned}
& \frac{\partial \overline{p_{D}}}{\partial x_{D}}=\alpha x_{D}^{\alpha-1}\{A \overbrace{I_{n}\left[\frac{\sqrt{z}}{\gamma} x_{D}^{\gamma}\right]}^{A 2}+B \overbrace{K_{n}\left[\frac{\sqrt{z}}{\gamma} x_{D} \gamma\right]}^{A 3}\} \\
& +x_{D}{ }^{\alpha}\left\{A(\overbrace{I_{n-1}\left[\frac{\sqrt{z}}{\gamma} x_{D} \gamma\right]}^{A 1}-\frac{n}{x_{D} \gamma} \frac{\gamma}{\sqrt{z}} \overbrace{I_{n}\left[\frac{\sqrt{z}}{\gamma} x_{D} \gamma\right]}^{A 2}) \sqrt{z} x_{D}^{\gamma-1}\right. \\
& \left.-B(\overbrace{K_{n-1}\left[\frac{\sqrt{z}}{\gamma} x_{D} \gamma\right]}^{A 1}+\frac{n}{x_{D} \gamma} \frac{\gamma}{\sqrt{z}} \overbrace{K_{n}\left[\frac{\sqrt{z}}{\gamma} x_{D} \gamma\right]}^{A 3}) \sqrt{z} x_{D}^{\gamma-1}\right\}
\end{aligned}
$$

In Eq. 3.42 we define some temporary variables (A1, A2 and A3) to assist with bookkeeping factorization of terms related to these bracket numbers leads to: 


$$
\begin{aligned}
\frac{\partial \overline{p_{D}}}{\partial x_{D}}= & x_{D}{ }^{\alpha+\gamma-1} \sqrt{z}\{A \overbrace{I_{n-1}\left[\frac{\sqrt{z}}{\gamma} x_{D}^{\gamma}\right]}^{A 1}-B \overbrace{K_{n-1}\left[\frac{\sqrt{z}}{\gamma} x_{D}^{\gamma}\right]}^{A 1}\} \\
& +\{A \overbrace{I_{n}\left[\frac{\sqrt{z}}{\gamma} x_{D} \gamma\right]}^{A 2}+B \overbrace{K_{n}\left[\frac{\sqrt{z}}{\gamma} x_{D}^{\gamma}\right]}^{A 3}\}\{\overbrace{\alpha x_{D}^{\alpha-1}-x_{D}^{\alpha} \frac{n}{x_{D} \gamma} \frac{\gamma}{\sqrt{z}} \sqrt{z} x_{D}^{\gamma-1}}^{A 4}\} \ldots . .
\end{aligned}
$$

Defining the term "A4" as shown in Eq. 3.43, we can then reduce this term as follows:

$$
\alpha x_{D}^{\alpha-1}-x_{D}^{\alpha} \frac{n}{x_{D} \gamma} \frac{\gamma}{\sqrt{z}} \sqrt{z} x_{D}^{\gamma-1}=\alpha x_{D}^{\alpha-1}-x_{D}^{\alpha-1} n \gamma
$$

Because $n \gamma=\frac{\theta+3-d}{\theta+2} \frac{\theta+2}{2}=\frac{\theta+3-d}{2}=\alpha$, substitution into Eq. 3.44 yields:

$$
\alpha x_{D}^{\alpha-1}-x_{D}^{\alpha-1} n \gamma=\alpha x_{D}^{\alpha-1}-x_{D}^{\alpha-1} \alpha=0
$$

The temporary variable A4 is then equal to zero; so Eq. 3.43 is simplified to the following form:

$$
\frac{d \overline{p_{D}}}{d x_{D}}=x_{D}^{\alpha+\gamma-1} \sqrt{z}\left\{A I_{n-1}\left[\frac{\sqrt{z}}{\gamma} x_{D}^{\gamma}\right]-B K_{n-1}\left[\frac{\sqrt{z}}{\gamma} x_{D}^{\gamma}\right]\right\}
$$

We need to determine the constants $A$ and $B$ to find the particular solution. Taking the Laplace Transform of both boundary conditions $3.36 \mathrm{~b}$ and $3.36 \mathrm{c}$, we have the following system:

$$
\left\{\begin{array}{c}
\lim _{x_{D} \rightarrow 1}\left(\frac{\partial \overline{p_{D}}}{\partial x_{D}}\right)=-\frac{1}{z} \\
\lim _{x_{D} \rightarrow L}\left(\frac{\partial \overline{p_{D}}}{\partial x_{D}}\right)=0
\end{array}\right.
$$

This is not a trivial calculation if conventional methods are attempted, but a solution can be readily obtained by using software with symbolic math capabilities, such as Mathematica 8 (Wolfram 2010). The following code shown in Figure 3.2 is used to solve the system: 


$$
\begin{aligned}
& \text { data }=\{\alpha>0, \gamma>0, n>1, z>0\} \\
& \text { PDgensol[1. } \left.\mathrm{x}_{\mathrm{B}}\right]:=x^{\alpha}\left(A \operatorname{BesselI}\left[n, \frac{\sqrt{z}}{\gamma} x^{\gamma}\right]+B \operatorname{BesselK}\left[n, \frac{\sqrt{z}}{\gamma} x^{\gamma}\right]\right)
\end{aligned}
$$

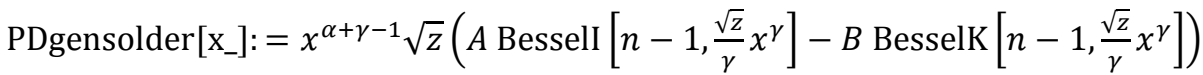

$$
\begin{aligned}
& \lim 1=\operatorname{Limit}[\operatorname{PDgensolder}[x], x \rightarrow 1 \text {, Assumptions } \rightarrow \text { data }]==-\frac{1}{z} \\
& \lim 2=\operatorname{Limit}[\text { PDgensolder }[x], x \rightarrow L \text {, Assumptions } \rightarrow \text { data }]==0 \\
& \text { sol }=\text { Solve }[\{\lim 1, \lim 2\},\{A, B\}, \text { Method } \rightarrow \text { Legacy }][[1]] \\
& \mathrm{PD}\left[\mathrm{x}_{-}\right]=\operatorname{PDgensol}[x] / . \text { sol }
\end{aligned}
$$

Figure 3.2 - Mathematica 8 code to solve Equ 3.46

Executing these commands, Mathematica 8 returns the following particular solution:

$$
\overline{p_{D}}\left(x_{D}, d, \theta, L, z\right)=x_{D}^{\alpha} \frac{I_{n}\left[\frac{x_{D}^{\gamma} \sqrt{z}}{\gamma}\right] K_{n-1}\left[\frac{L^{\gamma} \sqrt{z}}{\gamma}\right]+I_{n-1}\left[\frac{L^{\gamma} \sqrt{z}}{\gamma}\right] K_{n}\left[\frac{x_{D}^{\gamma} \sqrt{z}}{\gamma}\right]}{z^{\frac{3}{2}}\left(I_{n-1}\left[\frac{L^{\gamma} \sqrt{z}}{\gamma}\right] K_{n-1}\left[\frac{\sqrt{z}}{\gamma}\right]-I_{n-1}\left[\frac{\sqrt{z}}{\gamma}\right] K_{n-1}\left[\frac{L^{\gamma} \sqrt{z}}{\gamma}\right]\right)} .
$$

At the wellbore $\left(x_{D}=1\right)$, and Eq. 3.47 is reduced to the classic linear and radial flow cases:

- If we set $\left\{x_{D}=1, d=3, \theta=0\right\}$, then we obtain:

$$
\begin{aligned}
& \alpha=\frac{\theta+3-d}{2}=0 \\
& n=\frac{\theta+3-d}{\theta+2}=0 \\
& \gamma=\frac{\theta+2}{2}=1
\end{aligned}
$$

Additionally, the modified Bessel function of the second kind $K_{v}(z)$ is said to be even with respect to its parameter $v$ (Bowman 1958), that is to say

$$
K_{-v}(z)=K_{v}(z)
$$

Similarly, the modified Bessel function of the second kind $I_{v}(z)$ is even with respect to its parameter $v$ only if the parameter $v$ is an integer

$$
I_{-v}(z)=I_{v}(z)
$$


Thus, Eq. 3.47 reduces to the classic result for radial flow (Blasingame 2010b)

$$
\overline{P_{D}}\left(x_{D}=1, d=3, \theta=0, L, z\right)=\frac{I_{0}[\sqrt{z}] K_{1}[L \sqrt{z}]+I_{1}[L \sqrt{z}] K_{0}[\sqrt{z}]}{z^{3 / 2}\left(I_{1}[L \sqrt{z}] K_{1}[\sqrt{z}]-I_{1}[\sqrt{z}] K_{1}[L \sqrt{z}]\right)}
$$

- Similarly, we can also consider the case where we set $\left\{x_{D}=1, d=2, \theta=0\right\}$

$$
\begin{aligned}
& \alpha=\frac{\theta+3-d}{2}=\frac{1}{2} \\
& n=\frac{\theta+3-d}{\theta+2}=\frac{1}{2} \\
& \gamma=\frac{\theta+2}{2}=1
\end{aligned}
$$

In this case, Eq. 3.47 becomes

$$
\overline{p_{D}}\left(x_{D}=1, d=2, \theta=0, L, z\right)=\frac{I_{1 / 2}[\sqrt{z}] K_{1 / 2}[L \sqrt{z}]+I_{-1 / 2}[L \sqrt{z}] K_{1 / 2}[\sqrt{z}]}{z^{\frac{3}{2}}\left(I_{-1 / 2}[L \sqrt{z}] K_{1 / 2}[\sqrt{z}]-I_{-1 / 2}[\sqrt{z}] K_{1 / 2}[L \sqrt{z}]\right)}
$$

This expression requires further manipulation if it is to match the classic result of Blasingame (2010a) for linear flow. We use the following identities (Kreyszig 2005)

$$
\begin{aligned}
& I_{1 / 2}[x]=\sqrt{\frac{2}{\pi}} \frac{\sinh [x]}{\sqrt{x}} . \\
& I_{-1 / 2}[x]=\sqrt{\frac{2}{\pi}} \frac{\cosh [x]}{\sqrt{x}} . \\
& K_{1 / 2}[x]=\sqrt{\frac{\pi}{2}} \frac{e^{-x}}{\sqrt{x}}
\end{aligned}
$$

Substituting Eqs. 3.51a-3.51c into Eq. 3.50 yields:

$$
\begin{aligned}
& \overline{p_{D}}\left(x_{D}=1, d=2, \theta=0, L, z\right)=\frac{\sqrt{\frac{2}{\pi}} \frac{\sinh [\sqrt{z}]}{z^{\frac{1}{4}}} \sqrt{\frac{\pi}{2}} \frac{e^{-L \sqrt{z}}}{\sqrt{L} z^{\frac{1}{4}}}+\sqrt{\frac{2}{\pi}} \frac{\cosh [L \sqrt{z}]}{\sqrt{L} z^{\frac{1}{4}}} \sqrt{\frac{\pi}{2}} \frac{e^{-\sqrt{z}}}{z^{\frac{1}{4}}}}{z^{\frac{3}{2}}\left(\sqrt{\frac{2}{\pi}} \frac{\cosh [L \sqrt{z}]}{\sqrt{L} z^{\frac{1}{4}}} \sqrt{\frac{\pi}{2}} \frac{e^{-\sqrt{z}}}{z^{\frac{1}{4}}}-\sqrt{\frac{2}{\pi}} \frac{\cosh [\sqrt{z}]}{z^{\frac{1}{4}}} \sqrt{\frac{\pi}{2}} \frac{e^{-L \sqrt{z}}}{\sqrt{L} z^{\frac{1}{4}}}\right)} \\
& =\frac{\sinh [\sqrt{z}] e^{-L \sqrt{z}}+\cosh [L \sqrt{z}] e^{-\sqrt{z}}}{z^{\frac{3}{2}}\left(\cosh [L \sqrt{z}] e^{-\sqrt{z}}-\cosh [\sqrt{z}] e^{-L \sqrt{z})}\right.} .
\end{aligned}
$$


We now use the hyperbolic identities (Kreyszig 2005)

$$
\begin{aligned}
& \sinh [x]=\frac{e^{x}-e^{-x}}{2} . \\
& \cosh [x]=\frac{e^{x}+e^{-x}}{2} . \\
& \tanh [x]=\frac{\sinh [x]}{\cosh [x]} .
\end{aligned}
$$

Substituting Eqs. 3.53a and 3.53b into Eq. 3.52 yields

$$
\begin{aligned}
\overline{p_{D}}\left(x_{D}=1, d=2, \theta=0, L, z\right) & =\frac{\left(e^{\sqrt{z}}-e^{-\sqrt{z}}\right) e^{-L \sqrt{z}}+\left(e^{L \sqrt{z}}+e^{-L \sqrt{z}}\right) e^{-\sqrt{z}}}{z^{\frac{3}{2}}\left(\left(e^{L \sqrt{z}}+e^{-L \sqrt{z}}\right) e^{-\sqrt{z}}-\left(e^{\sqrt{z}}+e^{-\sqrt{z}}\right) e^{-L \sqrt{z}}\right)} \\
& =\frac{1}{z^{\frac{3}{2}}} \frac{e^{\sqrt{z}(L-1)}+e^{-\sqrt{z}(L-1)}}{e^{\sqrt{z}(L-1)}-e^{-\sqrt{z}(L-1)}} \\
& =\frac{1}{z^{\frac{3}{2}}} \frac{1}{\tanh [\sqrt{z}(L-1)]}
\end{aligned}
$$

Where Eq. 3.54 is identical to the Blasingame (2010a) expression for linear flow. As a note, $L=2$ must be used to match the reference result exactly.

\subsection{Numerical Validation}

In order to advance our hypothesis, the FDE needs to be validated, and numerical simulation is an ideal mechanism for this validation. We use a finite-volume black-oil reservoir simulator (Moridis and Cossio 2010) to create a simple linear 1D reservoir whose porosity and permeability change with distance from the wellbore, as stipulated in the FPPR (Eqs 3.1 and 3.2). For this validation we require a sufficiently fine space discretization (i.e., a sufficiently large number of cells $=1000$ ) to achieve a smooth porosity and permeability profile. Each cell represents a subdomain with its own (and different from all others) permeability and porosity. Furthermore, because it is not possible to have infinite reservoirs or infinite 
hydraulic properties in a simulator, an appropriately defined finite reservoir system is needed for comparison to the analytical solution of Eq. 3.47. The two possible options are either a no-flow or a constant-pressure outer boundary; we chose to use the no-flow outer boundary option for this purpose.

Numerical simulations were performed for 8 different cases, where the fractal parameters for these cases are detailed in Table 3.1. Additionally, the reservoir parameters common to all simulations are detailed in Table 3.2. A sketch of the reservoir is presented in Figure 3.3. Finally the permeability and porosity distributions with respect to distance are plotted in Figure 3.4 and Figure 3.5, respectively. There is excellent agreement between the analytical and numerical cases as shown in Figure 3.6.

In this section, we validate the analytical solution (Eq. 3.47) using numerical simulation. It is important to note that there is nothing "fractal" about our reservoir simulation - specifically, there are no chaotic processes, no randomly distributed network of fractures and no double-porosity assumptions. This leads us to suggest that we were successful in this validation effort not because the flow is fractal per se, but rather because both the FDE and the reservoir simulator solve equations based on the principle of conservation of mass. In short, we have solved exactly the same problem both analytically and numerically, providing a "proof-of-concept" for the FDE approach.

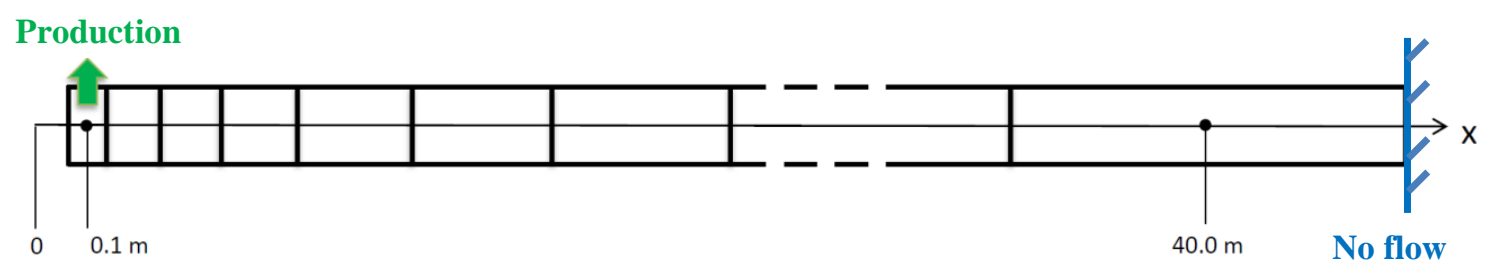

Figure 3.3 - Schematic of the 1D linear reservoir common to all cases. This reservoir has 1000 gridblocks in the X-direction, each with its own porosity and permeability, as stipulated in the FPPR. The permeability and porosity distributions with respect to $\mathrm{x}$, for all 8 cases, is shown in Figure 3.4 and Figure 3.5, respectively 
Table 3.1 - Parameters of the 8 cases to be simulated

\begin{tabular}{|c|c|c|c|c|c|c|c|}
\hline $\begin{array}{c}\text { Case } \\
\text { Number }\end{array}$ & $\begin{array}{c}d \\
(-)\end{array}$ & $\begin{array}{c}\theta \\
(-)\end{array}$ & $\begin{array}{c}k \text { at } x_{D}=1 \\
(\mathrm{md})\end{array}$ & $\begin{array}{c}k \text { at } x_{D}=\mathrm{L} \\
(\mathrm{md})\end{array}$ & $\begin{array}{c}\phi \text { at } x_{D}=1 \\
(\%)\end{array}$ & $\begin{array}{c}\phi \text { at } x_{D}=L \\
(\%)\end{array}$ & $\begin{array}{c}\text { Production } \\
\text { rate }(\mathrm{g} / \mathrm{s})\end{array}$ \\
\hline 1 & 2.0 & 0.0 & 1.0 & 1.00 & 0.1 & 0.10 & 0.5 \\
\hline 2 & 2.25 & 0.0 & 1.0 & 4.47 & 0.1 & 0.447 & 0.5 \\
\hline 3 & 2.50 & 0.0 & 1.0 & 20.00 & 0.1 & 2.00 & 0.8 \\
\hline 4 & 2.75 & 0.0 & 1.0 & 89.44 & 0.1 & 8.94 & 1.0 \\
\hline 5 & 3.0 & 0.0 & 1.0 & $4.00 \times 10^{2}$ & 0.1 & 40.00 & 1.0 \\
\hline 6 & 3.0 & -0.75 & 1.0 & $3.57 \times 10^{4}$ & 0.1 & 40.00 & 1.0 \\
\hline 7 & 1.969 & -0.7778 & 1.0 & 87.74 & 30.0 & 24.91 & 1.0 \\
\hline 8 & 1.661 & -0.323 & 1.0 & 0.91 & 5.0 & 0.65 & 1.0 \\
\hline
\end{tabular}

Table 3.2 - Reservoir and flow parameters common to all simulation cases

Parameter

$x_{w}$

$c_{o}$

$c_{r}$

$c_{T}$

$p_{\text {ini }}$

$\mu_{o}$

$\rho_{o}$

$\Delta x$

$\Delta y$

$\Delta z$

No. cells : $x$ direction

No. cells : $y$ direction

No. cells : $z$ direction
Value

0.1

0.0

$10^{-9}$

$10^{-9}$

$7.10^{7}$

$4.82 \times 10^{-4}$

745

linearly vary from $10^{-3}$ to 0.215

1

1

1000

1

1
Units

m

$\mathrm{Pa}^{-1}$

$\mathrm{Pa}^{-1}$

$\mathrm{Pa}^{-1}$

$\mathrm{Pa}$

Pa.s

$\mathrm{kg} \cdot \mathrm{m}^{-3}$

m

m

m 


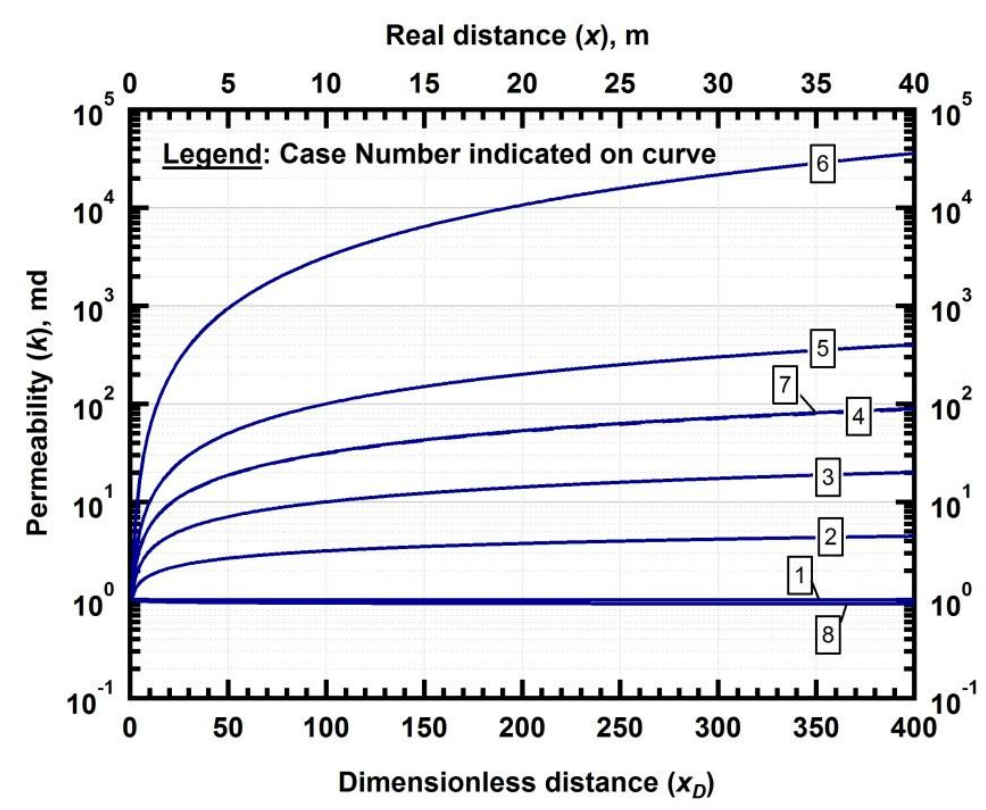

Figure 3.4 - Permeability vs. distance. The linear 1D reservoir shown in Figure 3.3 has a permeability that varies with distance from the wellbore, as stipulated by the FPPR (Eq. 3.1). The 8 cases that were simulated are listed in Table 3.1 are labeled accordingly.

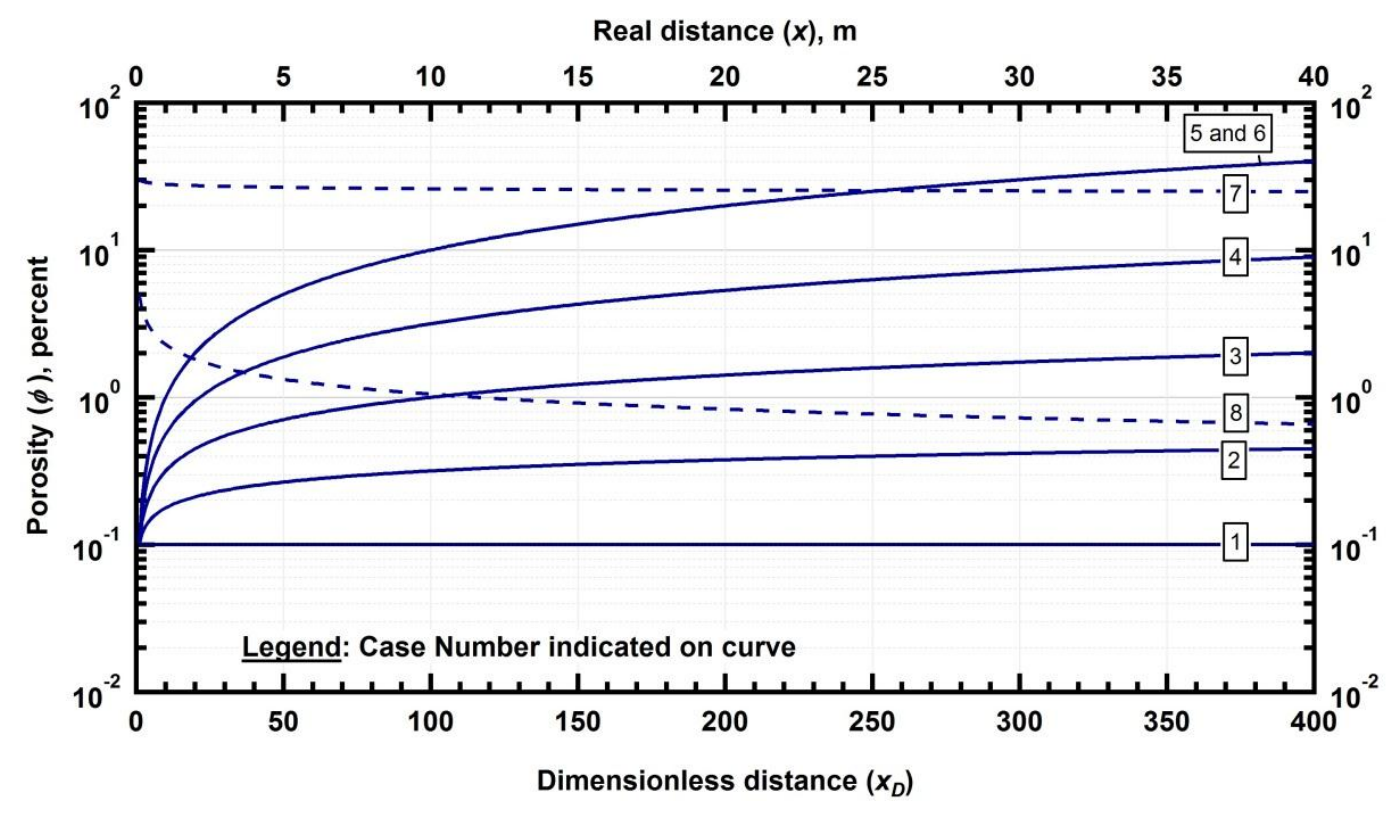

Figure 3.5 - Porosity vs. distance. The linear 1D reservoir shown in Figure 3.3 has a porosity that varies with distance from the wellbore, as stipulated by the FPPR (Eq. 3.2). The 8 cases that were simulated are listed in Table 3.1 are labeled accordingly. 


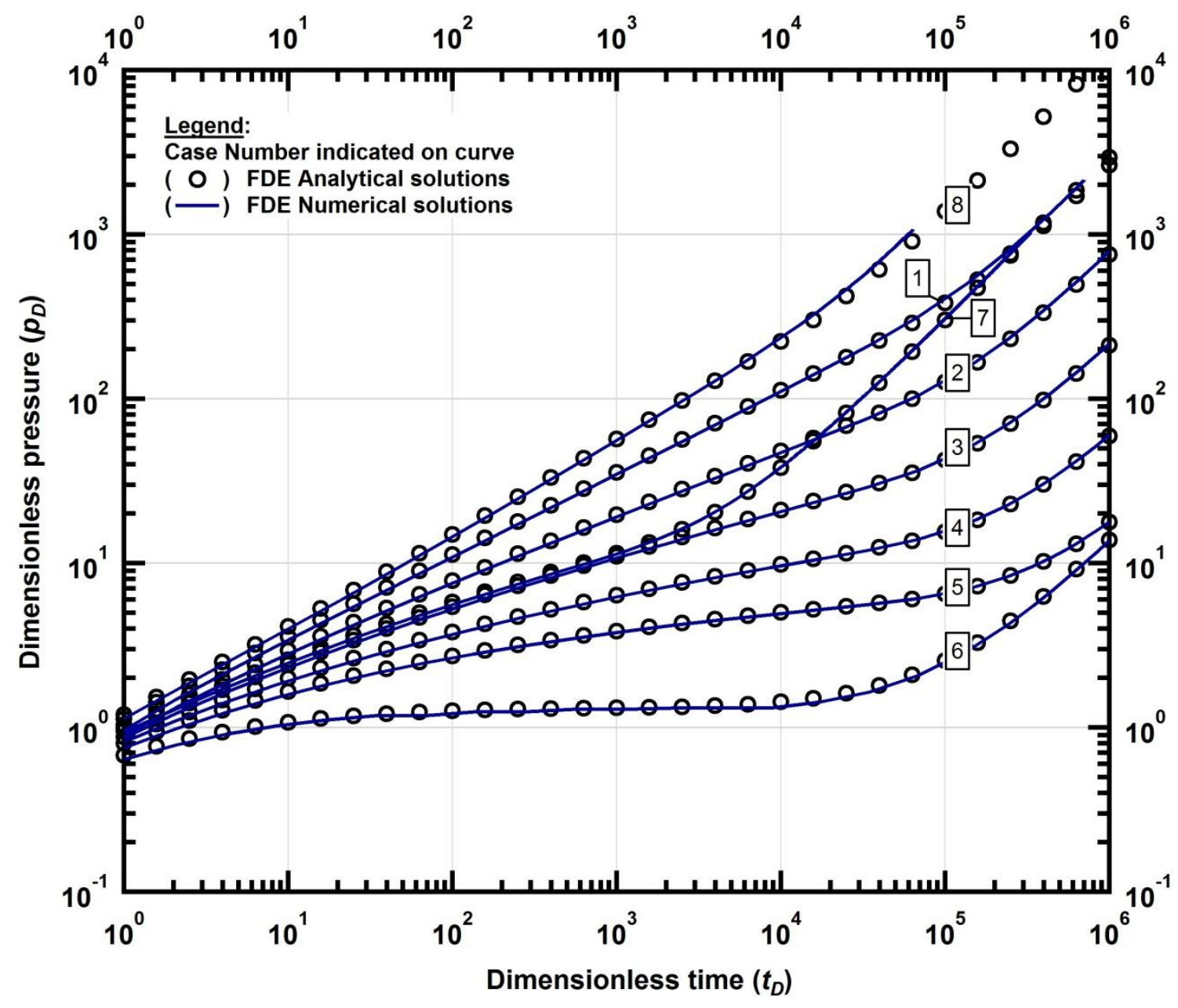

Figure 3.6 - Comparison between analytical and numerical results of the Fractal Diffusivity Equation. Note that Case 1 is equivalent to a Euclidean linear case, and Case 5 is equivalent to a Euclidean radial case. The excellent agreement in Case 5 validates numerically the equivalency proposed in Figure 3.1. 


\section{DEVELOPMENT OF THE FRACTAL-FRACTURE SOLUTION}

\subsection{Trilinear Flow Model with Fractal Modification}

In this thesis, our goal is to combine the trilinear flow model given by Lee and Brockenbrough (1986) with fractal theory in order to develop a fast and accurate semi-analytical solution for the problem of a producing well with a single vertical fracture that fully penetrates an infinite-acting homogeneous reservoir. We first note that fast and reliable solutions for this physical scenario already exist in the literature - Blasingame and Poe (1993) provide a "trilinear pseudo-radial solution" which is based on a coupling of the trilinear flow solution (which does not model radial flow) and the solution for a uniform flux/infinite conductivity vertical fracture (which does model pseudo-radial flow).

In our development of this semi-analytical solution, we believe that this is the first application of fractal theory to address a flow problem that is not related to naturally-fractured reservoirs. In demonstrating this development using the Fractal Diffusivity Equation, we hope to encourage its use in the development of solutions to currently intractable problems.

The basic premise of Lee and Brockenbrough (1986) is to idealize the flow into the hydraulic fracture by connecting the fracture to a system of sequentially connected 1D (linear) reservoirs, where this combination of 3 reservoirs (including the fracture) will produce a different flow regime. Each of these linear reservoirs is called a "region," and each has a governing diffusivity equation, and each reservoir communicates with each other through their common boundaries (which maintain common flux conditions). This scenario is depicted in Figure 4.1. 


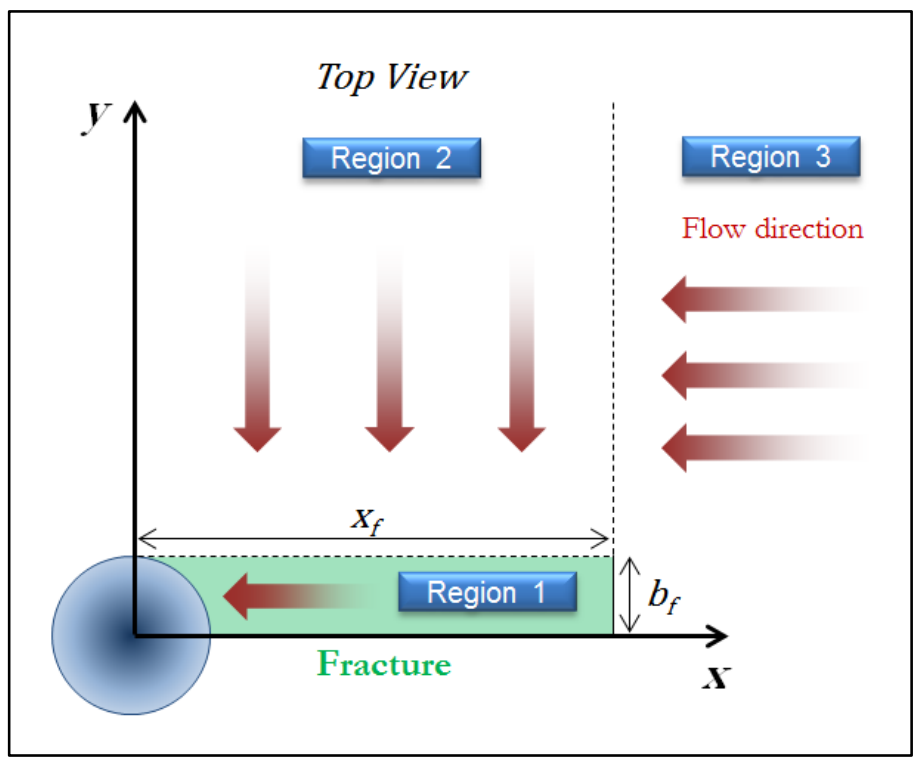

Figure 4.1 - Schematic of the trilinear flow concept as proposed by Lee and Brockenbrough (1986). Because of symmetry only a quadrant of the flow domain is considered. Region 3 flows in the $\mathrm{x}$ direction and meets Region 2 at the dashed lines at $x=x_{f}$; both Regions 2 and 3 involve formation flow. Region 2 flows in the y-direction and meets Region 1 at the dashed lines at $y=b_{f}$; Region 1 represents an idealized vertical fracture. Finally, the fracture flow of Region 1 feeds the wellbore, represented by the circle.

Mathematically, the trilinear flow system (with fractal geometry) is described as follows:

- Region 3 (formation flow):

$\frac{\partial^{2} p_{3 D}}{\partial x_{D}{ }^{2}}+\frac{d_{3}-\theta_{3}-2}{x_{D}} \frac{\partial p_{3 D}}{\partial x_{D}}=x_{D}^{\theta_{3}} \frac{\partial p_{3 D}}{\partial t_{D}}$

Initial condition:

$p_{3 D}=0$ when $t_{D}=0$

Inner Boundary condition: $\quad p_{3 D}=p_{2 D}$ when $x_{D}=1$

Outer Boundary Condition: $\quad p_{3 D}=0$ when $x_{D}=+\infty$

- Region 2 (formation flow):

$$
\frac{\partial^{2} p_{2 D}}{\partial y_{D}{ }^{2}}+\frac{d_{2}-\theta_{2}-2}{y_{D}} \frac{\partial p_{2 D}}{\partial y_{D}}+\left.y_{D}{ }^{\theta_{2}} \frac{\partial p_{3 D}}{\partial x_{D}}\right|_{x_{D}=1}=y_{D}{ }_{2} \frac{\partial p_{2 D}}{\partial t_{D}}
$$


Initial condition:

$$
p_{2 D}=0 \text { when } t_{D}=0 \text {. }
$$

Inner Boundary condition: $\quad p_{1 D}=p_{2 D}-S \frac{\partial p_{2 D}}{\partial y_{D}}$ when $y_{D}=1$

Outer Boundary Condition: $\quad p_{2 D}=0$ when $y_{D}=+\infty$

- Region 1 (fracture flow):

$\frac{\partial^{2} p_{1 D}}{\partial x_{D}{ }^{2}}+\frac{d_{1}-\theta_{1}-2}{x_{D}} \frac{\partial p_{1 D}}{\partial x_{D}}+\left.a x_{D}^{\theta_{1}} \frac{\partial p_{2 D}}{\partial y_{D}}\right|_{y_{D}=1}=x_{D}^{\theta_{1}} c_{1} \frac{\partial p_{1 D}}{\partial t_{D}}$

Initial Condition:

$$
p_{1 D}=0 \text { when } t_{D}=0 \text {. }
$$

Inner Boundary condition: $\left.\quad \frac{\partial p_{1 D}}{\partial x_{D}}\right|_{x_{D}=1}=b\left(1-C_{D f} \frac{\partial p_{w D}}{\partial t_{D}}\right)$

Outer Boundary Condition: $\left.\quad \frac{\partial p_{1 D}}{\partial x_{D}}\right|_{x_{D}=\mathrm{L}}=0$

The terms in these equations are defined as follows:

$$
p_{1 D}=\frac{k h\left(p_{i}-p_{1}\right)}{q B \mu} \quad \text { for oil in Region } 1 \text { (similar expressions hold true for Regions } 2 \text { and 3) }
$$

And the remaining terms are given as:

$$
\begin{array}{ll}
t_{D}=\frac{k}{\phi \mu c_{t} x_{f}^{2}} t & C_{1}=\frac{k \phi_{f} c_{f t}}{k_{f} \phi c_{t}} \\
x_{D}=\frac{x}{x_{f}}, y_{D}=\frac{y}{b_{f}} & a=\frac{2}{F_{c D}} \\
F_{C D}=\frac{k_{f} b_{f}}{k x_{f}} & b=-\frac{\pi}{F_{c D}} \\
C_{D f}=\frac{C}{2 \pi \phi c_{t} h x_{f}^{2}} &
\end{array}
$$

Essentially the only difference between the original Lee and Brockenbrough (1986) model and the present work is that we have replaced the Euclidean linear diffusivity equations, as stipulated in the original paper, 
by their fractal counterparts (i.e., Eqs. 4.1, 4.3 and 4.5). With this formulation, if we set $d_{3}=d_{2}=d_{1}=2$ and $\theta_{3}=\theta_{2}=\theta_{1}=0$, then we obtain the original Euclidean formulation. In Lee and Brockenbrough's (1986) original paper, the solution gave very good performance at early times (i.e., $\left.t_{D} \leq 1\right)-$ however; because of the well-known inability of the Lee and Brockenbrough (1986) solution to model pseudo-radial flow, this solution begins to fail at approximately $t_{D}=1$. Our hypothesis is that we can correctly capture pseudo-radial flow using the Fractal-Fracture Solution formulation that we proposed above. This will be an approximate/semi-analytical solution as we must calibrate the $d$ and $\theta$-parameters, but our expectation is that we will capture the appropriate flow regimes in the pressure and pressure derivative function.

\subsection{Analytic Derivation of the Open-Ended Fractal-Fracture Solution}

In this section we derive the "region" solutions for the fractal diffusivity relations given above. We begin with Region 3 (the outermost region) and work our way back to Region 1 (the innermost region).

- $\quad$ Region 3 (formation flow):

Taking the Laplace Transform of Eq. 4.1 and using the initial condition 4.2a yields

$$
\frac{\partial^{2} \overline{p_{3 D}}}{\partial x_{D}^{2}}+\frac{d_{3}-\theta_{3}-2}{x_{D}} \frac{\partial \overline{p_{3 D}}}{\partial x_{D}}=x_{D}^{\theta_{3}} z \overline{p_{3 D}}
$$

Following the procedure discussed in Section 3.3, the general solution to Eq. 4.7 is

$$
\overline{p_{3 D}}\left(x_{D}, z\right)=x_{D}^{\alpha_{3}}\left\{A_{3} I_{n_{3}}\left[\frac{\sqrt{z}}{\gamma_{3}} x_{D}^{\gamma_{3}}\right]+B_{3} K_{n_{3}}\left[\frac{\sqrt{z}}{\gamma_{3}} x_{D}^{\gamma_{3}}\right]\right\}
$$

In all cases (here and throughout the thesis), the following variables are real constants:

$$
n_{i}=\frac{\theta_{i}+3-d_{i}}{\theta_{i}+2} \quad \alpha_{i}=\frac{\theta_{i}+3-d_{i}}{2} \quad \gamma_{i}=\frac{\theta_{i}+2}{2}
$$

To determine the constants $A_{3}$ and $B_{3}$, we utilize the Laplace transform of the two boundary conditions (Eqs. 4.2b and 4.2c) and solve the following system for $A_{3}$ and $B_{3}$. This Laplace transform of the boundary conditions is given as: 


$$
\left\{\begin{array}{l}
\lim _{x_{D} \rightarrow 1}\left(\overline{p_{3 D}}\right)=\overline{p_{2 D}} \\
\lim _{x_{D} \rightarrow+\infty}\left(\overline{p_{3 D}}\right)=0
\end{array}\right.
$$

The Mathematica 8 code to solve this problem is shown in Figure 4.2:

$$
\begin{aligned}
& \text { data3 }=\{\alpha 3>-10, \mathrm{n} 3>-10, \gamma 3>0, z>0\}
\end{aligned}
$$

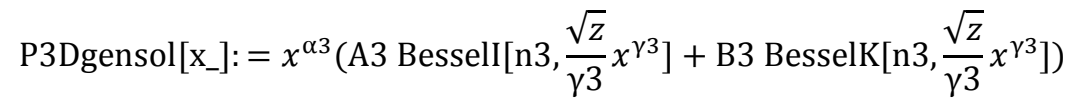

$$
\begin{aligned}
& \lim 1=\operatorname{Limit}[\mathrm{P} 3 \operatorname{Dgensol}[x], x \rightarrow 1]==\mathrm{P} 2 \mathrm{D} \\
& \lim 2=\operatorname{Limit}[\mathrm{P} 3 \operatorname{Dgensol}[x], x \rightarrow \infty \text {, Assumptions } \rightarrow \text { data3 }]==0 \\
& \text { sol1 }=\text { Solve[\{lim1, } \lim 2\},\{\text { A3, B3 }\}, \text { Method } \rightarrow \text { Legacy }][[1]] \\
& \mathrm{P} 3 \mathrm{D}\left[\mathrm{x}_{-}\right]=\mathrm{P} 3 \text { Dgensol }[x] / \text {.sol1 }
\end{aligned}
$$

Figure 4.2 - Mathematica 8 code for solving Eq. 4.9.

The particular solution to Eq. 4.7 is given by the Mathematica 8 software as

$$
\overline{p_{3 D}}\left(x_{D}, z\right)=\overline{p_{2 D}} x_{D}^{\alpha_{3}} \frac{K_{n_{3}}\left[\frac{\sqrt{z}}{\gamma_{3}} x_{D}^{\gamma_{3}}\right]}{K_{n_{3}}\left[\frac{\sqrt{z}}{\gamma_{3}}\right]}
$$

To determine the derivative of the pressure equation (Eq. 4.10) we follow the step-by-step process discussed below.

From the properties of Bessel functions (Bowman 1958),

$$
\frac{d\left(K_{n}[f(x)]\right)}{d x}=-f^{\prime}(x)\left\{K_{n-1}[f(x)]+\frac{n}{f(x)} K_{n}[f(x)]\right\}
$$

Application of Eq. 4.11 to Eq. 4.10 yields:

$$
\left.\frac{\partial \overline{p_{3 D}}}{\partial x_{D}}\right|_{x_{D}=1}=-\overline{p_{2 D}}\left\{\sqrt{z}\left(\frac{K_{n_{3}-1}\left[\frac{\sqrt{z}}{\gamma_{3}}\right]}{K_{n_{3}}\left[\frac{\sqrt{z}}{\gamma_{3}}\right]}+\frac{n_{3} \gamma_{3}}{\sqrt{z}}\right)-\alpha_{3}\right\} .
$$


However, $n_{3} \gamma_{3}=\frac{3-\left(d_{3}-\theta_{3}\right)}{\theta_{3}+2} \frac{\theta_{3}+2}{2}=\frac{3-\left(d_{3}-\theta_{3}\right)}{2}=\alpha_{3}$,

Which, after appropriate substitutions, yields

$$
\left.\frac{\partial \overline{p_{3 D}}}{\partial x_{D}}\right|_{x_{D}=1}=-\overline{p_{2 D}} \Omega(z)
$$

Where:

$$
\Omega(z)=\frac{K_{n_{3}-1}\left[\frac{2 \sqrt{z}}{\theta_{3}+2}\right]}{K_{n_{3}}\left[\frac{2 \sqrt{z}}{\theta_{3}+2}\right]} \sqrt{z}
$$

- Region 2 (formation flow):

Taking the Laplace transform of Eq. 4.3 and applying the initial condition (Eq. 4.4a), the substituting Eq. 4.13 into Eq. 4.3 gives us:

$$
\frac{\partial^{2} \overline{p_{2 D}}}{\partial y_{D}{ }^{2}}+\frac{d_{2}-\theta_{2}-2}{y_{D}} \frac{\partial \overline{p_{2 D}}}{\partial y_{D}}=y_{D} \theta_{2}(z+\Omega) \overline{p_{2 D}}
$$

Eqs. 4.7 and 4.14 differ only in that the $z$-term of the RHS of Eq. 4.7 is now $(z+\Omega)$. This term does not depend on $y_{D}$, so it may be treated as a constant. This yields the following form:

$$
\overline{p_{2 D}}\left(y_{D}, z\right)=y_{D}^{\alpha_{2}}\left\{A_{2} I_{n_{2}}\left[\frac{\sqrt{z+\Omega}}{\gamma_{2}} y_{D}^{\gamma_{2}}\right]+B_{2} K_{n_{2}}\left[\frac{\sqrt{z+\Omega}}{\gamma_{2}} y_{D}^{\gamma_{2}}\right]\right\}
$$

Following the process described in Section 3.3, the derivative of the general solution is given as:

$$
\frac{\partial \overline{p_{2 D}}}{\partial y_{D}}=y_{D}{ }^{\alpha_{2}+\gamma_{2}-1} \sqrt{z+\Omega}\left\{A_{2} I_{n_{2}-1}\left[\frac{\sqrt{z+\Omega}}{\gamma_{2}} y_{D} \gamma_{2}\right]-B_{2} K_{n_{2}-1}\left[\frac{\sqrt{z+\Omega}}{\gamma_{2}} y_{D} \gamma_{2}\right]\right\}
$$

To determine the constants $A_{2}$ and $B_{2}$ we again take the Laplace transform of the two boundary conditions (Eqs. 4.4b and 4.4c) and solve the resulting system for $A_{2}$ and $B_{2}$. The Laplace transform of the boundary conditions is given as: 


$$
\left\{\begin{array}{c}
\lim _{y_{D} \rightarrow 1}\left(\overline{p_{2 D}}-S \frac{\partial \overline{p_{2 D}}}{\partial y_{D}}\right)=\overline{p_{1 D}} \\
\lim _{y_{D} \rightarrow+\infty}\left(\overline{p_{2 D}}\right)=0
\end{array}\right.
$$

The Mathematica 8 code to solve this is shown in Figure 4.3:

$$
\begin{aligned}
& \text { data } 2=\{\alpha>0, \gamma>0, n>1, z>0, \Omega>0\} \\
& \text { P2Dgensol[_[_]: }=y^{\alpha}\left(\mathrm{A} 2 \operatorname{BesselI}\left[n, \frac{\sqrt{z+\Omega}}{\gamma} y^{\gamma}\right]+\mathrm{B} 2 \operatorname{BesselK}\left[n, \frac{\sqrt{z+\Omega}}{\gamma} y^{\gamma}\right]\right) \\
& \text { P2Dgensolder[y_]: }=y^{\alpha+\gamma-1} \sqrt{z+\Omega}\left(\mathrm{A} 2 \operatorname{BesselI}\left[n-1, \frac{\sqrt{z+\Omega}}{\gamma} y^{\gamma}\right]\right. \\
& \left.-\mathrm{B} 2 \text { BesselK }\left[n-1, \frac{\sqrt{z+\Omega}}{\gamma} y^{\gamma}\right]\right) \\
& \lim 3=\operatorname{Limit}[\mathrm{P} 2 \operatorname{Dgensol}[y], y \rightarrow \infty \text {, Assumptions } \rightarrow \text { data2 }]=0 \\
& \lim 4=\operatorname{Limit}[\mathrm{P} 2 \mathrm{Dgensol}[y]-S \text { P2Dgensolder}[y], y \rightarrow 1 \text {, Assumptions } \rightarrow \text { data2] } \\
& ==\mathrm{P} 1 \mathrm{D} \\
& \text { sol2 }=\text { Solve }[\{\lim 3, \lim 4\},\{\text { A2, B2 }\} \text {, Method } \rightarrow \text { Legacy }][[1]] \\
& \mathrm{P} 2 \mathrm{D}\left[\mathrm{y}_{-}\right]=\mathrm{P} 2 \mathrm{Dgensol}[y] / \text {. sol2 }
\end{aligned}
$$

Figure 4.3 - Mathematica 8 code for solving Eq. 4.17.

Using the Mathematica 8 software, we obtain the particular solution to Eq. 4.14 as:

$$
\overline{p_{2 D}}\left(y_{D}, z\right)=\frac{\overline{p_{1 D}} y_{D}^{\alpha_{2}} K_{n_{2}}\left[\frac{\sqrt{z+\Omega}}{\gamma_{2}} y_{D}^{\gamma_{2}}\right]}{S \sqrt{z+\Omega} K_{n_{2}-1}\left[\frac{\sqrt{z+\Omega}}{\gamma_{2}}\right]+K_{n_{2}}\left[\frac{\sqrt{z+\Omega}}{\gamma_{2}}\right]}
$$

Using the identity given by Eq. 4.11, we obtain the derivative at $y_{D}=1$, which is required for the solution in Region 1.

$$
\left.\frac{\partial \overline{p_{2 D}}}{\partial y_{D}}\right|_{y_{D}=1}=\frac{\overline{p_{1 D}}\left(\alpha_{2} K_{n_{2}}\left[\frac{\sqrt{z+\Omega}}{\gamma_{2}}\right]-\sqrt{z+\Omega}\left\{K_{n_{2}-1}\left[\frac{\sqrt{z+\Omega}}{\gamma_{2}}\right]+\frac{n_{2}}{\sqrt{z+\Omega}} K_{n_{2}}\left[\frac{\sqrt{z+\Omega}}{\gamma_{2}}\right]\right\}\right)}{S \sqrt{z+\Omega} K_{n_{2}-1}\left[\frac{\sqrt{z+\Omega}}{\gamma_{2}}\right]+K_{n_{2}}\left[\frac{\sqrt{z+\Omega}}{\gamma_{2}}\right]}
$$

- $\quad$ Region 1 (fracture flow): 
Taking the Laplace Transform of Eq. 4.5, using the initial condition 4.6a, and substituting in Eq. 4.19 in 4.5 yields

$$
\frac{\partial^{2} \overline{p_{1 D}}}{\partial x_{D}{ }^{2}}+\frac{d_{1}-\theta_{1}-2}{x_{D}} \frac{\partial \overline{p_{1 D}}}{\partial x_{D}}=\psi^{2} \overline{p_{1 D}} x_{D} \theta_{1},
$$

Where:

$$
\psi=\left[\frac{-a\left(\alpha_{2} K_{n_{2}}\left[\frac{\sqrt{z+\Omega}}{\gamma_{2}}\right]-\sqrt{z+\Omega}\left\{K_{n_{2}-1}\left[\frac{\sqrt{z+\Omega}}{\gamma_{2}}\right]+\frac{n_{2}}{\sqrt{z+\Omega}} K_{n_{2}}\left[\frac{\sqrt{z+\Omega}}{\gamma_{2}}\right]\right\}\right)}{S \sqrt{z+\Omega} K_{n_{2}-1}\left[\frac{\sqrt{z+\Omega}}{\gamma_{2}}\right]+K_{n_{2}}\left[\frac{\sqrt{z+\Omega}}{\gamma_{2}}\right]}+C_{1} Z\right]^{1 / 2}
$$

And we note that $\Omega$ is defined as previously by Eq. 4.13. Similar to our procedures for Eqs. 4.7 and 4.14, we obtain the general solution to Eq. 4.20 as:

$$
\overline{p_{1 D}}\left(x_{D}, z\right)=x_{D}^{\alpha_{1}}\left\{A_{1} I_{n_{1}}\left[\frac{\psi}{\gamma_{1}} x_{D}^{\gamma_{1}}\right]+B_{1} K_{n_{1}}\left[\frac{\psi}{\gamma_{1}} x_{D}^{\gamma_{1}}\right]\right\}
$$

The corresponding derivative is obtained by following the same procedure described earlier:

$$
\frac{\partial \overline{p_{1 D}}}{\partial x_{D}}=x_{D}^{\alpha_{1}+\gamma_{1}-1} \psi\left\{A_{1} I_{n_{1}-1}\left[\frac{\psi}{\gamma_{1}} x_{D}^{\gamma_{1}}\right]-B_{1} K_{n_{1}-1}\left[\frac{\psi}{\gamma_{1}} x_{D}^{\gamma_{1}}\right]\right\}
$$

To determine the constants $A_{1}$ and $B_{1}$ we again take the Laplace transform of the two boundary conditions (Eqs. 4.6b and 4.6c) and solve the resulting system for $A_{1}$ and $B_{1}$. The Laplace transform of the boundary conditions is given as:

$$
\left\{\begin{array}{c}
\lim _{x_{D} \rightarrow 1}\left(\frac{\partial \overline{p_{1 D}}}{\partial x_{D}}\right)=\frac{1}{z} b\left(1-C_{D f} \overline{p_{w D}} z^{2}\right) \\
\lim _{x_{D} \rightarrow L}\left(\frac{\partial \overline{p_{1 D}}}{\partial x_{D}}\right)=0
\end{array}\right.
$$

where $L$ in Eq. 4.23 is the dimensionless distance to the no-flow outer boundary.

The Mathematica 8 code used to solve this system is shown in Figure 4.4: 


$$
\begin{aligned}
& \text { P1Dgensol }\left[\mathrm{z}_{-}, x_{-}\right]:=x^{\alpha}\left(\mathrm{A} 1 \text { BesselI }\left[\mathrm{n} 1, \frac{\psi}{\gamma} x^{\gamma}\right]+\text { B1 BesselK }\left[\mathrm{n} 1, \frac{\psi}{\gamma} x^{\gamma}\right]\right) \\
& \text { P1Dgender }\left[\mathrm{z}_{-} x_{-}\right]:=x^{\alpha+\gamma-1} \psi\left(\text { A1 BesselI }\left[n-1, \frac{\psi}{\gamma} x^{\gamma}\right]-\text { B1 BesselK }\left[n-1, \frac{\psi}{\gamma} x^{\gamma}\right]\right) \\
& \lim 5=\operatorname{Limit}[\mathrm{P} 1 \mathrm{Dgender}[z, x], x \rightarrow 1]==\frac{1}{z} b\left(1-\operatorname{CDf} \operatorname{PWD} z^{2}\right) \\
& \lim 6=\operatorname{Limit}[\mathrm{P} 1 \mathrm{Dgender}[z, x], x \rightarrow L]==0 \\
& \text { sol3 }=\text { Solve[\{lim5, lim6\}, \{A1, B1 }\}, \text { Method } \rightarrow \text { Legacy }] \\
& \mathrm{P} 1 \mathrm{D}\left[\mathrm{z}_{-}, \mathrm{x}_{-}\right]=\mathrm{P} 1 \mathrm{Dgensol}[z, x] / \text {. sol3 } \\
& \mathrm{P} 1 \mathrm{D}[z, 1]
\end{aligned}
$$

Figure 4.4 - Mathematica 8 code for solving Eq. 4.23

Using the Mathematica 8 software, we obtain the particular solution to Eq. 4.20 as:

$$
\begin{aligned}
& \overline{p_{1 D}}\left(C_{D f}, F_{C D}, S, C_{1}, x_{D}=1, L, z\right) \\
& =\frac{b\left(C_{D f} \overline{p_{W D}} z^{2}-1\right)}{z \psi} \frac{\left(I_{n_{1}}\left[\frac{\psi}{\gamma_{1}}\right] K_{n_{1}-1}\left[\frac{L^{\gamma_{1}} \psi}{\gamma_{1}}\right]+I_{n_{1}-1}\left[\frac{L \gamma_{1} \psi}{\gamma_{1}}\right] K_{n_{1}}\left[\frac{\psi}{\gamma_{1}}\right]\right)}{\left(I_{n_{1}-1}\left[\frac{L \gamma_{1} \psi}{\gamma_{1}}\right] K_{n_{1}-1}\left[\frac{\psi}{\gamma_{1}}\right]-I_{n_{1}-1}\left[\frac{\psi}{\gamma_{1}}\right] K_{n_{1}-1}\left[\frac{L \gamma_{1} \psi}{\gamma_{1}}\right]\right)} .
\end{aligned}
$$

For convenience, we define the following $\Delta$ term as a "lumped variable" so that Eq. 4.24 is more compact and easier to manipulate:

$$
\Delta=\frac{I_{n_{1}-1}\left[\frac{{ }^{\gamma_{1} \psi}}{\gamma_{1}}\right] K_{n_{1}-1}\left[\frac{\psi}{\gamma_{1}}\right]-I_{n_{1}-1}\left[\frac{\psi}{\gamma_{1}}\right] K_{n_{1}-1}\left[\frac{{ }^{\gamma_{1} \psi}}{\gamma_{1}}\right]}{I_{n_{1}}\left[\frac{\psi}{\gamma_{1}}\right] K_{n_{1}-1}\left[\frac{{ }^{\gamma_{1} \psi}}{\gamma_{1}}\right]+I_{n_{1}-1}\left[\frac{{ }^{\gamma_{1}} \psi}{\gamma_{1}}\right] K_{n_{1}}\left[\frac{\psi}{\gamma_{1}}\right]}
$$

For the time being, we consider $L$ to be an independent variable. In Section 4.3 we discuss Eq. 4.25 and $L$ further. Defining $\overline{p_{1 D}}\left(x_{D}=1\right)=\overline{p_{w D}}$, we obtain the following solution using Eq. 4.24 for the wellbore pressure in the trilinear model.

$$
\overline{p_{w D}}=\frac{b\left(C_{D f} \overline{p_{w D}} z^{2}-1\right)}{z \psi \Delta}
$$

Or, 
$z \psi \Delta \overline{p_{W D}}=b C_{D f} \overline{p_{W D}} z^{2}-b$

Isolating $\overline{p_{w D}}$, we have:

$\overline{p_{W D}}\left(z \psi \Delta-b C_{D f} z^{2}\right)=-b$

Solving for $\overline{p_{w D}}$, we obtain:

$$
\overline{p_{W D}}=\frac{b}{z\left(b C_{D f^{z}}-\psi \Delta\right)}
$$

For the case where $S=0 ; C_{l}=0 ; C_{D f}=0$, Eq. 4.26 simplifies to

$$
\overline{p_{w D}}\left(F_{c D}, Z\right)=\frac{\pi}{F_{c D} Z \psi \Delta}
$$

Eq. 4.27 can be inverted numerically from the Laplace space using the Gaver-Stehfest algorithm (1970).

The $\psi$ term, previously defined in Eq. 4.20, can also be simplified. Eliminating the $S$ and $C_{l}$ terms, we have:

$$
\begin{aligned}
\psi & =\left[\frac{\left.-a\left(\alpha_{2} K_{n_{2}}\left[\frac{\sqrt{Z+\Omega}}{\gamma_{2}}\right]-\sqrt{z+\Omega}\left\{K_{n_{2}-1}\left[\frac{\sqrt{Z+\Omega}}{\gamma_{2}}\right]+\frac{n_{2}}{\sqrt{z+\Omega}} K_{n_{2}}\left[\frac{\sqrt{Z+\Omega}}{\gamma_{2}}\right]\right\}\right)\right]^{\frac{1}{2}}}{K_{n_{2}}\left[\frac{\sqrt{z+\Omega}}{\gamma_{2}}\right]}\right. \\
& =\left[-a\left(\alpha_{2}-\sqrt{z+\Omega} \frac{K_{n_{2}-1}\left[\frac{\sqrt{z+\Omega}}{\gamma_{2}}\right]}{K_{n_{2}}\left[\frac{\sqrt{z+\Omega}}{\gamma_{2}}\right]}-n_{2}\right)\right]^{\frac{1}{2}}
\end{aligned}
$$

Expanding and reducing the $\alpha-n$ term, we have: 


$$
\begin{aligned}
\alpha-n & =\frac{\theta+3-d}{2}-\frac{\theta+3-d}{\theta+2} \\
& =\frac{(\theta+3-d)(\theta+2)-2(\theta+3-d)}{2(\theta+2)} \\
& =\frac{\theta^{2}+2 \theta+3 \theta+6-d \theta-2 d-2 \theta-6+2 d}{2(\theta+2)} \\
& =\frac{\theta^{2}-d \theta+3 \theta}{2(\theta+2)} \\
& =\frac{\theta(\theta-d+3)}{2(\theta+2)} \\
& =\frac{\theta}{2} n
\end{aligned}
$$

Our final form of the expression for the $\psi$-function, for use in Eq. 4.27, is given as:

$$
\psi=\left\{\frac{1}{F_{c D}}\left(2 \sqrt{z+\Omega} \frac{K_{n_{2}-1}\left[\frac{2 \sqrt{z+\Omega}}{\theta_{2}+2}\right]}{K_{n_{2}}\left[\frac{2 \sqrt{z+\Omega}}{\theta_{2}+2}\right]}-\theta_{2} n_{2}\right)\right\}^{1 / 2}
$$

\subsection{Selection and Derivation of Study Scenarios}

At this point, we have successfully redeveloped the trilinear flow solution and coupled this with the components of the Fractal Diffusivity Equations (in the Laplace domain). This formulation led to the introduction of 6 (six) additional variables for the trilinear flow problem (specifically: $d_{1}, d_{2}, d_{3}, \theta_{1}, \theta_{2}$

and $\theta_{3}$ ). There is no independent mechanism for estimating these parameters - so we must calibrate our proposed solution to a "standard" solution for the case of a well with a single finite-conductivity vertical fracture producing in an infinite-acting homogeneous reservoir.

For this calibration we have selected the Cinco-Meng (1988) solution, and we will estimate the $d_{1}, d_{2}, d_{3}$, $\theta_{1}, \theta_{2}$ and $\theta_{3}$ parameters by numerical optimization. As a precautionary measure, we have chosen to simplify the problem because optimization of six parameters may invite issues of non-uniqueness and substantially increased computational cost. As such, we consider 3 different "scenarios," where we leave a particular Region(s) in their linear (non-fractal) form — these scenarios are listed in Table 4.1. 
Table 4.1 - Scenarios to be optimized

\begin{tabular}{|c|c|c|c|c|c|c|}
\hline Scenario & Region 1 & Region 2 & Region 3 & $\begin{array}{c}\text { Unknowns to be } \\
\text { optimized }\end{array}$ & $\begin{array}{c}t_{D} \text { range } \\
\text { considered }\end{array}$ & $\begin{array}{l}F_{c D} \text { range } \\
\text { considered }\end{array}$ \\
\hline 1 & Linear & Fractal & Removed & $d_{2}$ and $\theta_{2}$ & $10^{-6}$ to $10^{7}$ & $10^{-1}$ to $10^{4}$ \\
\hline 2 & Linear & Fractal & Fractal & $d_{2}, d_{3}, \theta_{2}$ and $\theta_{3}$ & $10^{-6}$ to $10^{7}$ & $10^{-1}$ to $10^{4}$ \\
\hline 3 & Fractal & Fractal & Fractal & $d_{1}, d_{2}, d_{3}, \theta_{1}, \theta_{2}$ and $\theta_{3}$ & $10^{-6}$ to $10^{7}$ & $10^{-1}$ to $10^{4}$ \\
\hline
\end{tabular}

Eq. 4.27 describes the "master" scenario where all three Regions are "fractal" — this case is designated "Scenario 3." As shown in Table 4.1, Scenarios 1 and 2 are simplified versions of Scenario 3. We will develop the "master" scenario first, then work our way down to the simpler versions.

\section{Scenario 3:}

Scenario 3 is derived in Section 4.2, and its solution is given by Eq. 4.27. We have yet to discuss the $L$ value (the dimensionless distance to the boundary) which is present in the $\Delta$ term in Eq. 4.25 . In our formulation of the trilinear model using the Fractal Diffusivity Equation, we state that implicitly the porosity and permeability vary with distance $(x)$ according to power-law relations (Eqs. 3.1 and 3.2). As a consequence, we cannot define the inner boundary condition at $x_{D}=0$ (as Lee and Brockenbrough (1986) did) because this imposes exactly zero porosity and zero permeability at that point, which is physically inconsistent.

In our study we have defined Region 1 as being between $x_{D}=1$ and $x_{D}=L$. This is not an issue as the physical problem is exactly the same, but it has been defined mathematically in a different way. Specifically, we must establish the value of $L$ such that $\Delta=\tanh [\psi]$ when Region 1 is Euclidean, that is to say when $\left\{d_{1}=2, \theta_{1}=0\right\}$.

If we set $\left\{d_{1}=2, \theta_{1}=0\right\}$ in Eq. 4.25 , then $n_{1}=\frac{1}{2}$ and $\gamma_{1}=1$ which yields: 


$$
\Delta=\frac{I_{-\frac{1}{2}}[L \psi] K_{\frac{1}{2}}[\psi]-I_{-\frac{1}{2}}[\psi] K_{\frac{1}{2}}[L \psi]}{I_{-\frac{1}{2}}[L \psi] K_{\frac{1}{2}}[\psi]+I_{\frac{1}{2}}[\psi] K_{\frac{1}{2}}[L \psi]} .
$$

Using the following identities (Wolfram 2010):

$$
\begin{aligned}
& I_{1 / 2}[x]=\sqrt{\frac{2}{\pi}} \frac{\sinh [x]}{\sqrt{x}} \\
& I_{-1 / 2}[x]=\sqrt{\frac{2}{\pi}} \frac{\cosh [x]}{\sqrt{x}} \\
& K_{1 / 2}[x]=\sqrt{\frac{\pi}{2}} \frac{e^{-x}}{\sqrt{x}} \\
& \sinh [x]=\frac{e^{x}-e^{-x}}{2} \\
& \cosh [x]=\frac{e^{x}+e^{-x}}{2} \\
& \tanh [x]=\frac{\sinh [x]}{\cosh [x]}=\frac{e^{x}-e^{-x}}{e^{x}+e^{-x}},
\end{aligned}
$$

We substitute Eqs. 4.30a-4.30f into Eq. 4.29, and we obtain:

$$
\begin{aligned}
& \Delta=\frac{\sqrt{\frac{2}{\pi}} \frac{\cosh [L \psi]}{\sqrt{L \psi}} \sqrt{\frac{\pi}{2}} \frac{e^{-\psi}}{\sqrt{\psi}}-\sqrt{\frac{2}{\pi}} \frac{\cosh [\psi]}{\sqrt{\psi}} \sqrt{\frac{\pi}{2}} \frac{e^{-L \psi}}{\sqrt{L \psi}}}{\sqrt{\frac{2}{\pi}} \frac{\cosh [L \psi]}{\sqrt{L \psi}} \sqrt{\frac{\pi}{2}} \frac{e^{-\psi}}{\sqrt{\psi}}+\sqrt{\frac{2}{\pi}} \frac{\sinh [\psi]}{\sqrt{\psi}} \sqrt{\frac{\pi}{2}} \frac{e^{-L \psi}}{\sqrt{L \psi}}} \\
& =\frac{\cosh [L \psi] e^{-\psi}-\cosh [\psi] e^{-L \psi}}{\cosh [L \psi] e^{-\psi}+\sinh [\psi] e^{-L \psi}} \\
& =\frac{\frac{e^{L \psi}+e^{-L \psi}}{2} e^{-\psi}-\frac{e^{\psi}+e^{-\psi}}{2} e^{-L \psi}}{\frac{e^{L \psi}+e^{-L \psi}}{2} e^{-\psi}+\frac{e^{\psi}-e^{-\psi}}{2} e^{-L \psi}} \\
& =\frac{e^{\psi(L-1)}-e^{-\psi(L-1)}}{e^{\psi(L-1)}+e^{-\psi(L-1)}} \\
& =\tanh [\psi(L-1)]
\end{aligned}
$$

Therefore, we require that $L \equiv 2$ to be consistent with the trilinear flow model (where this is the same requirement we had for Eq. 3.54). 


\section{Scenario 2:}

In this Scenario, Regions 2 and 3 remain as they were derived in Section 4.2 - however; Region 1 is now treated as Euclidean (i.e., non-fractal), and as was just established, we set $L=2$ in the $\Delta$ term (Eq. 4.31). Therefore, Region 2 is only different from Region 3 in that

$$
\Delta=\tanh [\psi]
$$

Furthermore, $d_{1}$ and $\theta_{1}$ are no longer relevant variables and are set to $\left\{d_{1}=2, \theta_{1}=0\right\}$.

\section{Scenario 1:}

In Scenario 1 we further simplify the problem by eliminating Region 3 altogether (i.e., $p_{3 D}=0$ at all times). Tracking this term in Eq. 4.3, it implies that we remove $\Omega$ from Eq. 4.14. Additionally, $d_{3}$ and $\theta_{3}$ are set to 0 .

As a summary, the solution schemes for each scenario are given in Table 4.2. In all cases the skin factor $(S)$, the dimensionless wellbore storage coefficient $\left(C_{D f}\right)$, and the fracture storage factor $\left(C_{l}\right)$ were set to 0 .

\subsection{Discussion of Optimization Strategy}

In the process of combining the Fractal Diffusivity Equations into the trilinear flow solutions, we have introduced 6 (six) unknowns into the problem $\left(d_{1}, d_{2}, d_{3}, \theta_{1}, \theta_{2}\right.$ and $\left.\theta_{3}\right)$. At this point it is not clear as to whether or not these unknowns are constant, nor what variables the unknowns should be correlated against (although an obvious variable of correlation is the fracture conductivity). At this stage, our strategy is to numerically optimize these parameters $\left(d_{1}, d_{2}, d_{3}, \theta_{1}, \theta_{2}\right.$ and $\left.\theta_{3}\right)$ for each individual $F_{c D}$ case, and then to graphically compare each parameter to $F_{c D}$ to establish whether a single-variable correlation is appropriate. 
Table 4.2 - Analytical solutions for all scenarios

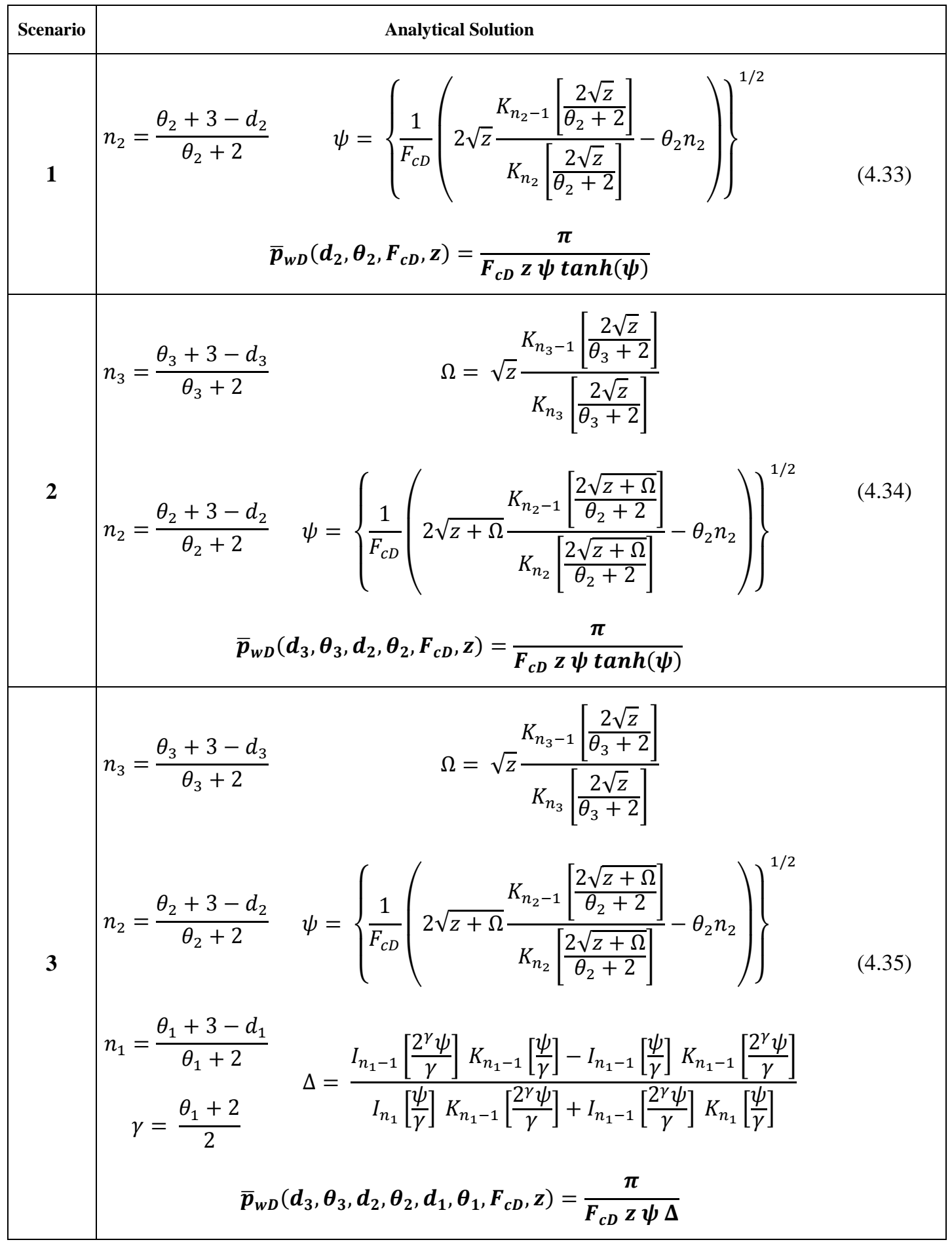


As mentioned earlier in this thesis, we have selected the Cinco-Meng (1988) solution as the "standard" against which we will correlate the fractal-based, trilinear flow solution. This Cinco-Meng (1988) solution is given as:

$$
\begin{aligned}
& \bar{p}_{w D}(z)-\frac{1}{2} \sum_{i=1}^{n}\left\{\bar{q}_{f D i}(z) \int_{x_{D i}}^{x_{D i+1}}\left[K_{0}\left(x_{D j}-x^{\prime}\right) \sqrt{z}+K_{0}\left(x_{D j}+x^{\prime}\right) \sqrt{z}\right] d x^{\prime}\right\} \\
& +\frac{\pi}{\left(k_{f} b_{f}\right)_{D}}\left\{\sum_{i=1}^{j-1}\left[\left(\frac{(\Delta x)^{2}}{2}+\Delta x\left(x_{D j}-i \Delta x\right)\right) \bar{q}_{f D i}(z)\right]+\frac{(\Delta x)^{2}}{8} \bar{q}_{f D j}(z)\right\}=\frac{\pi x_{D j}}{\left(k_{f} b_{f}\right)_{D} z}, \ldots \ldots \ldots . .
\end{aligned}
$$

And the "flux condition" is given by:

$$
\Delta x \sum_{i=1}^{n} \bar{q}_{f D i}(z)=\frac{1}{z}
$$

Where Eqs. 4.36a and 4.36b imply the following system of equations:

$$
\left[A_{i j}\right]\left[\begin{array}{c}
\bar{q}_{f D i}(z) \\
\bar{p}_{w D}(z)
\end{array}\right]=\left[B_{j}\right]
$$

Where the dimensionless pressure solution in the real (time) domain is obtained via numerical inversion of the $\bar{p}_{w D}(z)$ solution given in the Laplace domain. We note that, as a standard, the Cinco-Meng solution (1988) does provide a physically and mathematically rigorous treatment of the problem in the Laplace domain, but it is a discretized solution that is cumbersome, is complex to set up, and is computationally very expensive. Consequently, the Cinco-Meng solution is not well suited to history matching applications.

In order to calibrate the $d$ and $\theta$-values using the Cinco-Meng (1988) solution as a standard, we constructed a FORTRAN code (Chapman 2008) to perform the numerical optimization. This program incorporates the Levenberg-Marquardt optimization algorithm (Moré et al. 1984) coupled to the FractalFracture Solution (i.e., our Fracture Diffusivity Equations combined with the trilinear flow solution). A flowchart of this optimization process is depicted in Figure 4.5. 
Our optimization/calibration process begins with an initial guess for each of the values of the unknown fractal parameters; we then compute the Fractal-Fracture Solution using these values and compare the results to the Cinco-Meng Solution. In order to define an "objective function" for optimization, we must first define which function(s) shall be our basis - in our case we consider both the pressure function and the pressure derivative function, coupled by a defined weighting of each function. For this research, we have defined our objective function $\left(O_{F}\right)$ as:

$$
O_{F}=A \frac{\left|p_{W D \cdot F F S}-p_{w D \cdot C M S}\right|}{p_{w D \cdot C M S}}+B \frac{\left|p^{\prime}{ }_{W D \cdot F F S}-p^{\prime}{ }_{W D \cdot C M S}\right|}{p^{\prime}{ }_{W D \cdot C M S}}
$$

Where the individual components in Eq. 4.37 are defined as:

- $p_{w D \cdot F F S}$ is the dimensionless wellbore pressure (Fractal-Fracture Solution).

- $p_{w D \cdot C M S}$ is the dimensionless wellbore pressure (Cinco-Meng (1988) solution).

- $p_{w D \cdot F F S}^{\prime}$ is the dimensionless wellbore pressure derivative (Fractal-Fracture Solution).

- $p^{\prime}{ }_{w D \cdot C M S}$ is the dimensionless wellbore pressure derivative (Cinco-Meng (1988) solution).

- $A$ and $B$ are the weighting coefficients for the pressure and pressure derivative errors

The optimization process is terminated when the Levenberg-Marquardt algorithm determines via a specified tolerance that the objective function cannot be further minimized. We note that the objective function takes into account both the pressure and pressure derivative functions, but it is not immediately obvious what values their respective weighting coefficients $A$ and $B$ should have - as such, we considered 5 different cases of weighting coefficients, as outlined in Table 4.3. 
Table 4.3 - Evaluated weight coefficients for objective function

\begin{tabular}{|c|c|c|}
\hline Case & $p_{D}$ weight $-A$ & $p_{D}{ }^{\prime}$ weight $-B$ \\
\hline 1 & $0 \%$ & $100 \%$ \\
\hline 2 & $25 \%$ & $75 \%$ \\
\hline 3 & $50 \%$ & $50 \%$ \\
\hline 4 & $75 \%$ & $25 \%$ \\
\hline 5 & $100 \%$ & $0 \%$ \\
\hline
\end{tabular}

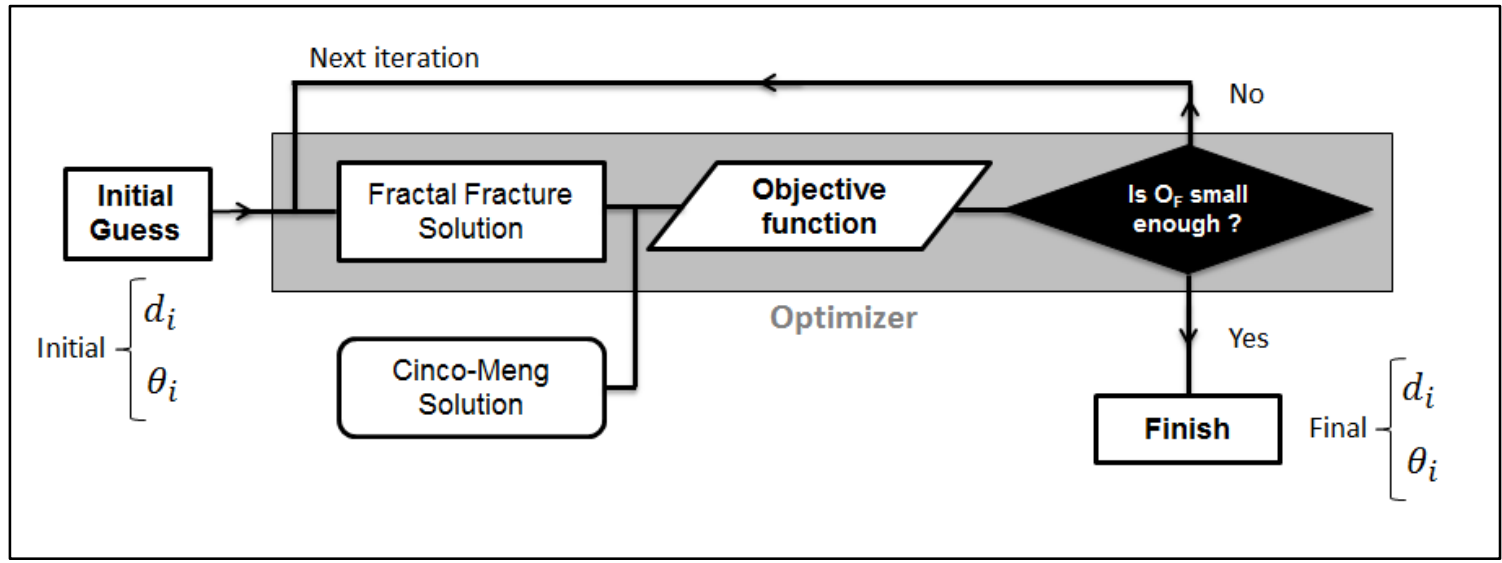

Figure 4.5 - Optimization process of the fractal parameters in the FFS. We begin with an initial guess of the values of the unknown fractal parameters, compute the Fractal-Fracture Solution with them, compare the results to the Cinco-Meng Solution, and attempt to optimize the parameter value by minimizing an objective function. The optimization is terminated when the Levenberg-Marquardt algorithm determines that the objective function $O_{F}$ cannot be further minimized.

All five weighting cases were tested for a low conductivity case (Figure 4.6), and high conductivity case (Figure 4.7) - and based on the performance of these cases we chose $A=0.25$ and $B=0.75$ as the most appropriate weighting factors. 


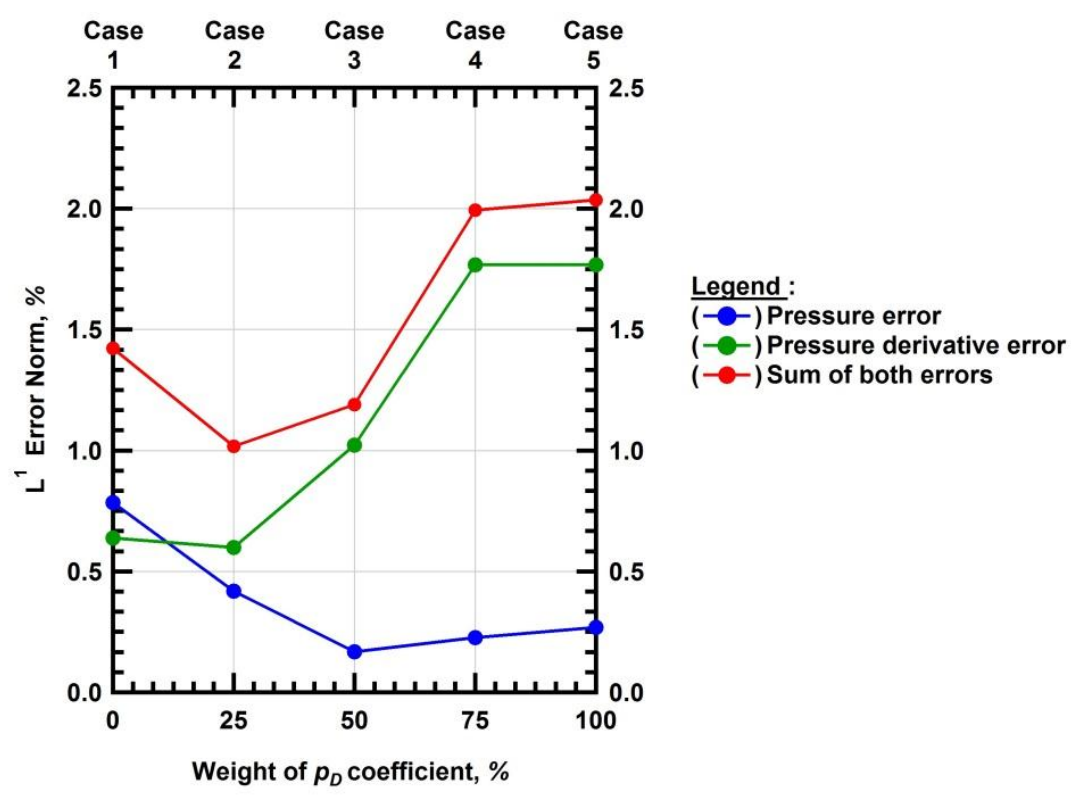

Figure 4.6 - Evaluation of five different objective functions for a low conductivity $\left(F_{c D}=0.5\right)$ case.

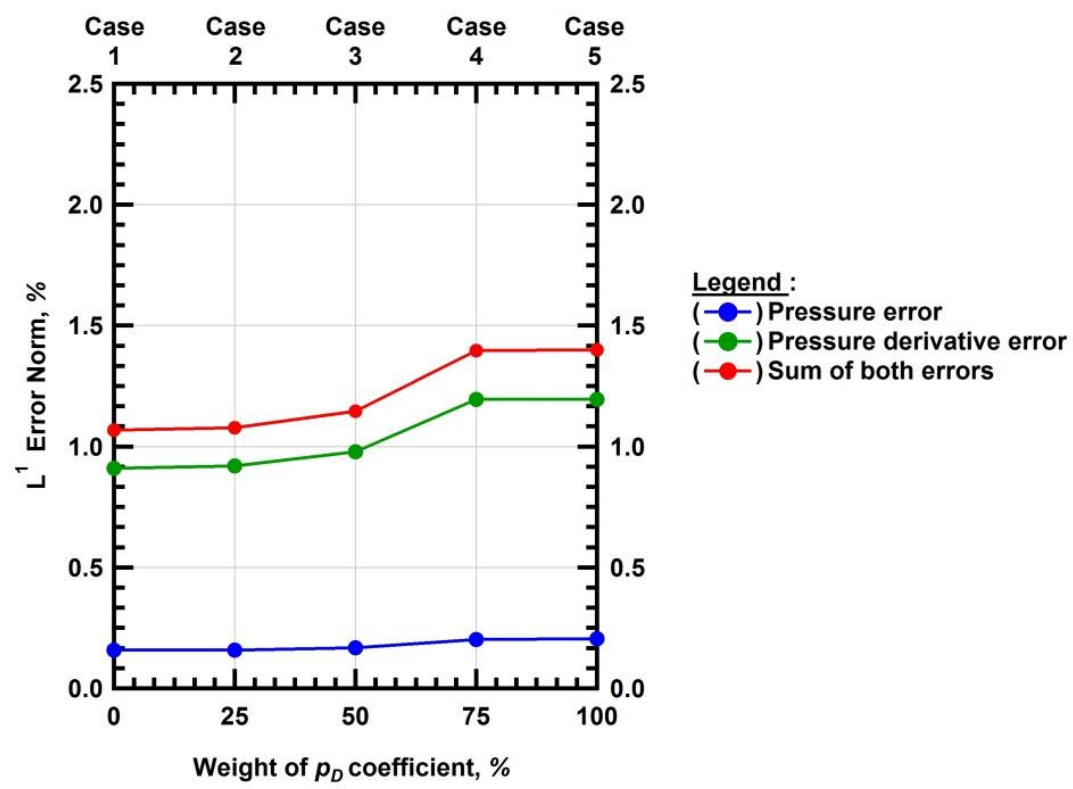

Figure 4.7 - Evaluation of five different objective functions for a high conductivity $\left(F_{c D}=10^{4}\right)$ case. 


\subsection{Optimization Results}

Using the calibration/optimization approach we were able to match the Cinco-Meng Solution (1988) satisfactorily with all three Scenarios (Table 4.1) over 13 log cycles of dimensionless time by using the optimizer (Figure 4.5). Figure 4.8 shows the $\mathrm{L}^{1}$ relative error norm of pressure versus the fracture conductivity for each optimized scenario. Inspection of Figure 4.8 and Table 4.2 leads to the following conclusions:

- Scenario 1 is by far the least accurate (most simple form/analytical solution).

- Scenario 3 is the most accurate (most complex form/analytical solution).

- Scenario 2 lies between the other two scenarios in terms of both solution complexity and accuracy.

- All scenarios fail for $F_{c D}<0.5$, and this should be the lower limit of applicability.

- Scenarios 2 and 3 are essentially equivalent for practical purposes ( $\mathrm{L}^{1}$ below $0.3 \%$ ).

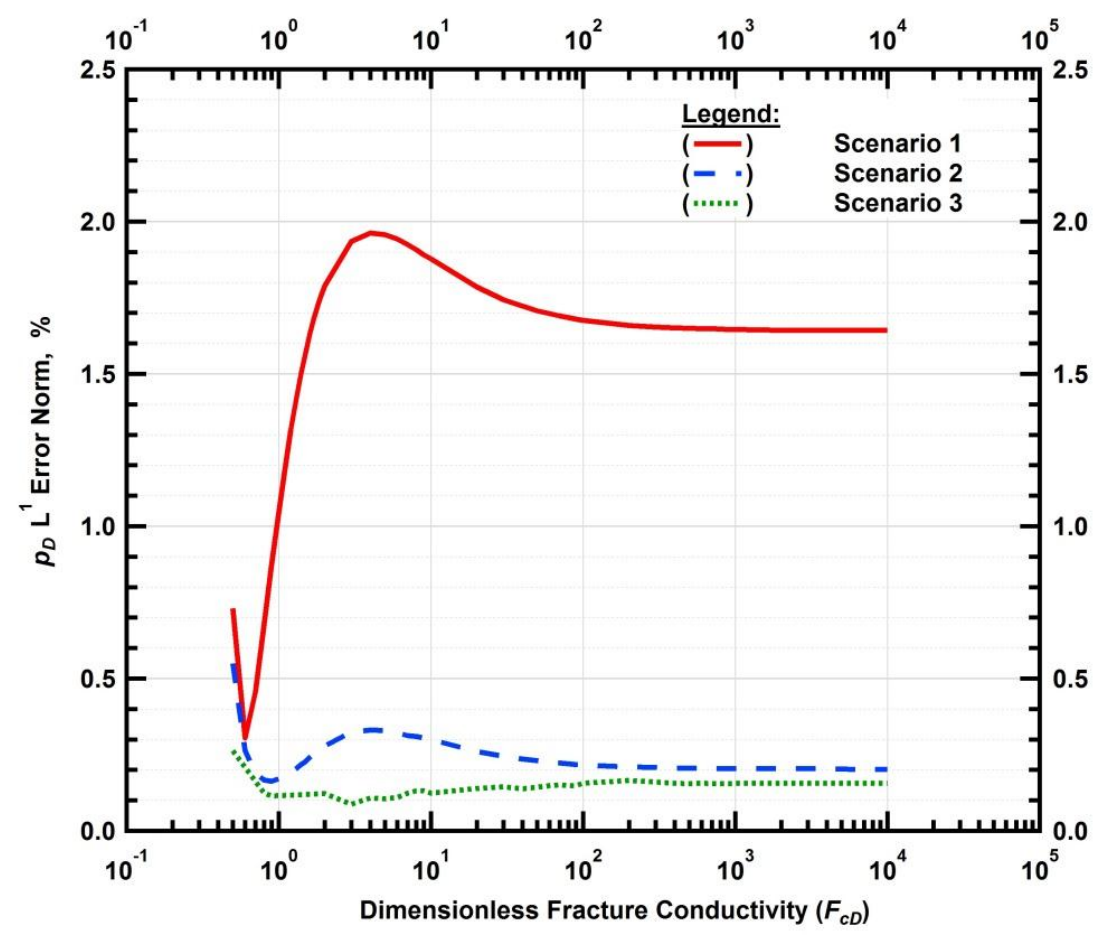

Figure 4.8 - The Fractal-Fracture Solution was derived analytically for the three Scenarios considered in Table 4.1. The solutions of Table 4.2 were each numerically optimized to match the Cinco-Meng (1988) solution. The $\mathrm{L}^{1}$ relative error norm (in percent) is plotted against fracture conductivity for each of these scenarios. 
Figure 4.9 shows the numerically optimized fractal parameters for Scenario 2. It is obvious that the curves are smooth and lend themselves to an approximation by a closed-form equation (this task will be performed in Section 5.1). Similarly, Figure 4.10 and Figure 4.11 show the optimized $d$ and $\theta$ parameters for Scenario 3, respectively. We note that in this case the curves are relatively smooth from $F_{c D}=10^{4}$ to $F_{c D}=3-$ for $F_{c D}<3$ the high number of fractal parameters ( 6 in this case) begin to cause nonuniqueness problems. This means that more than one combination of parameters may yield an acceptable answer, and this yields inconsistency in the parameters as $F_{c D}$ decreases.

The very high accuracy of these low conductivity results is offset by the fact that it is very hard to establish unique correlations for low conductivity values (this will be discussed in detail in Section 5.3). We also note that for Scenarios 2 and 3, all parameters remain constant for $F_{c D}>10^{3}$, which suggests we can extrapolate to $F_{c D}=\infty$ using values obtained for $F_{c D}=10^{4}$ (this would be a reasonable assumption).

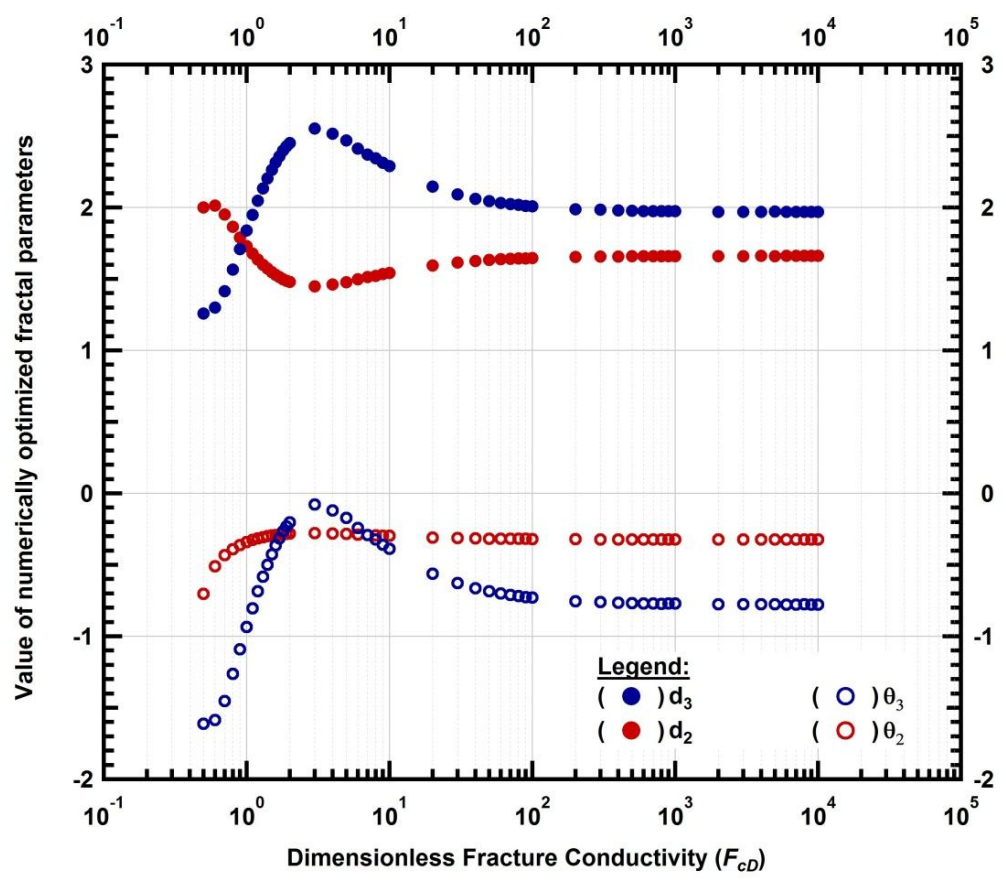

Figure 4.9 - Values of optimized fractal parameters versus the dimensionless fracture conductivity $\left(F_{c D}\right)$. The Fractal-Fracture Solution (Scenario 2 ) was numerically optimized to obtain values for the 4 fractal parameters in order to match the Cinco-Meng (1988) solution. Note that all curves are well-behaved and should lend themselves to approximations by smooth, closed form functions. 




Figure 4.10 - Numerically optimized fractal parameters for Scenario 3 ( $d$-parameters).

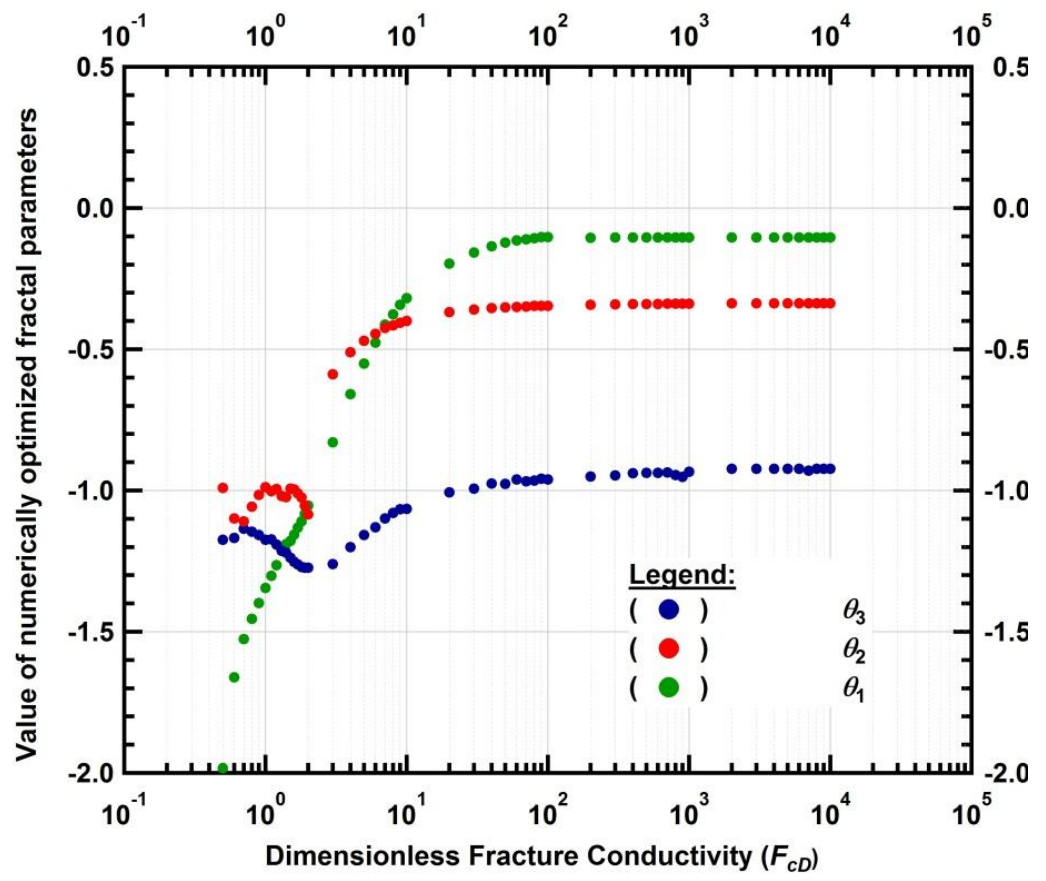

Figure 4.11 - Numerically optimized fractal parameters for Scenario 3 ( $\theta$-parameters). 


\section{FRACTAL-FRACTURE SOLUTION CORRELATIONS}

\subsection{Scenario 2: Parameter Correlations}

We begin with our efforts to optimize Scenario 2 (Region 1 (the fracture) non-fractal, Regions 2 and 3 fractal - Table 4.1) as this was our initial focus case, and has become our "most practical" scenario in terms of balancing solution accuracy and complexity. It was our impression at the time that the fracture (Region 1) would not need to be fractal — as will be discussed, there is some advantage in accuracy to deploy Region 1 as fractal (i.e., Scenario 3), but this incremental improvement in accuracy for Scenario 3 comes a cost in both complexity and solution uniqueness (i.e., the more complex the solution, the less unique it became).

For Scenario 2, we note that the optimized fractal parameters (see Figure 4.9) are well behaved when correlated against the dimensionless fracture conductivity $\left(F_{c D}\right)$ - as such, we believe it is possible to establish univariate correlations (i.e., $y$ versus $x$, where $\left.x=F_{c D}\right)$ for each fractal parameter $\left(d_{2}, d_{3}, \theta_{2}\right.$ and $\theta_{3}$ ). In order to develop these correlations, we employed the use of the TableCurve $2 D$ software (Systat 2012) which provides a nearly infinite library of possible data models, fitted and ranked statistically for a given regression of $y$ versus $x$.

Our correlations for Scenario 2 were constructed as follows:

- $\theta_{2}=f\left(F_{c D}\right): \theta_{2}$ is a unique function of $F_{c D}$

- $d_{2}=f\left(F_{c D}\right): d_{2}$ is a unique function of $F_{c D}$

- $d_{3}=f\left(d_{2}\right): d_{3}$ is a defined function of $d_{2}$

- $\theta_{3}=f\left(d_{3}\right): \quad \theta_{3}$ is a defined function of $d_{3}$

There may be seem to be a contradiction between correlating the parameters for Region $2\left(d_{2}\right.$ and $\left.\theta_{2}\right)$ in terms of the dimensionless fracture conductivity $\left(F_{c D}\right)$, but then defining the parameters for Region $3\left(d_{3}\right.$ and $\theta_{3}$ ) in terms of the parameters for Region 2 ( $d_{3}$ directly, and $\theta_{3}$ indirectly). However; these definitions for $d_{3}$ and $\theta_{3}$ arose from our correlation efforts and we believe that there may be a physical basis for these correlations (but this is not explored in our present work). For this work, our efforts have focused on 
establishing robust and accurate correlations for the fractal parameters (in this case: $d_{2}, d_{3}, \theta_{2}$ and $\theta_{3}$ ); and we believe that we have created (at the very least) a practical solution for application of the FractalFracture Solution (FFS) for this scenario.

The parameter correlations for Scenario 2 are provided below:

- $\theta_{2}=f\left(F_{c D}\right): \theta_{2}$ is a unique function of $F_{c D}$

$$
\theta_{2}=\frac{A_{1}+C_{1} x+E_{1} x^{2}+G_{1} x^{3}}{1+B_{1} x+D_{1} x^{2}+F_{1} x^{3}}\left(\text { where } x=\log _{10}\left(F_{c D}\right)\right)
$$

The following coefficients were determined using the TableCurve 2D software (Systat 2012):

$$
\begin{array}{ll}
A_{1}=-0.34048432 & E_{1}=-0.34553019 \\
B_{1}=1.918772436 & F_{1}=3.154995757 \\
C_{1}=-0.26952048 & G_{1}=-0.98791385 \\
D_{1}=0.433916281 &
\end{array}
$$

The data-model correlation for this case is shown in Figure 5.1.

- $d_{2}=f\left(F_{c D}\right): d_{2}$ is a unique function of $F_{c D}$

$$
d_{2}=\frac{A_{2}+C_{2} x+E_{2} x^{2}+G_{2} x^{3}}{1+B_{2} x+D_{2} x^{2}+F_{2} x^{3}}\left(\text { where } x=\log _{10}\left(F_{c D}\right)\right)
$$

The following coefficients were determined using the TableCurve 2D software (Systat 2012):

$$
\begin{array}{lll}
A_{2}=1.732300052 & E_{2}=4.547075660 \\
B_{2}=0.145343775 & F_{2}=1.016405890 \\
C_{2}=-1.00458904 & G_{2}=1.566926538 \\
D_{2}=2.270990983 &
\end{array}
$$

The data-model correlation for this case is shown in Figure 5.2. 


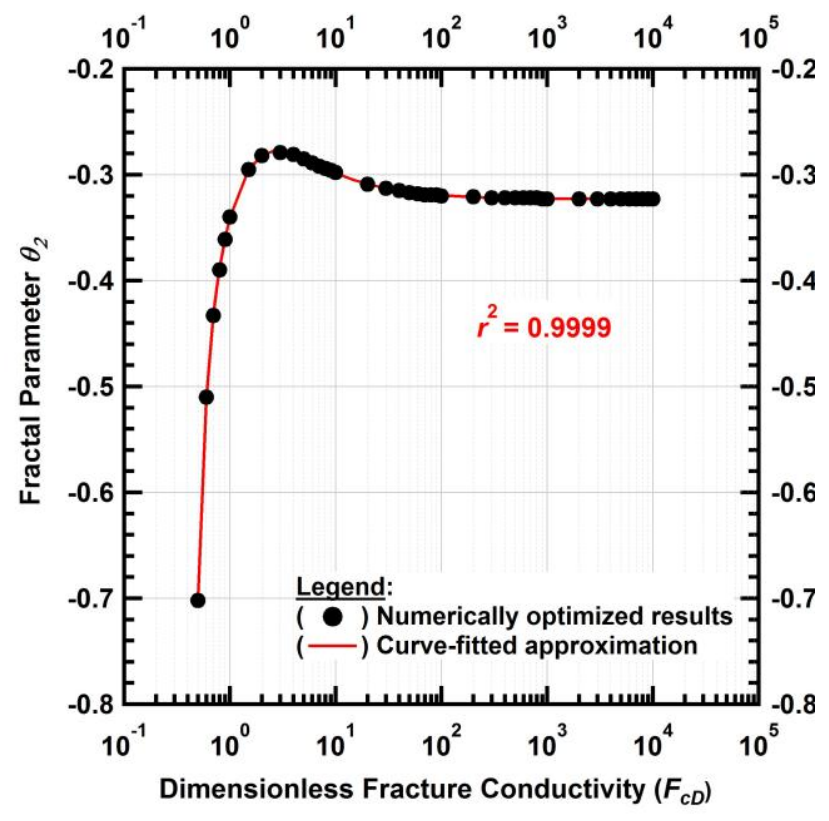

Figure 5.1 - Parameter $\theta_{2}$ versus dimensionless fracture conductivity $F_{c D}$. Each black circle represents the optimized $\theta_{2}$ for a given $F_{c D}$ value. The red line is the model approximation. The parameter $\theta_{2}$ is correlated solely in terms of $F_{c D}$.

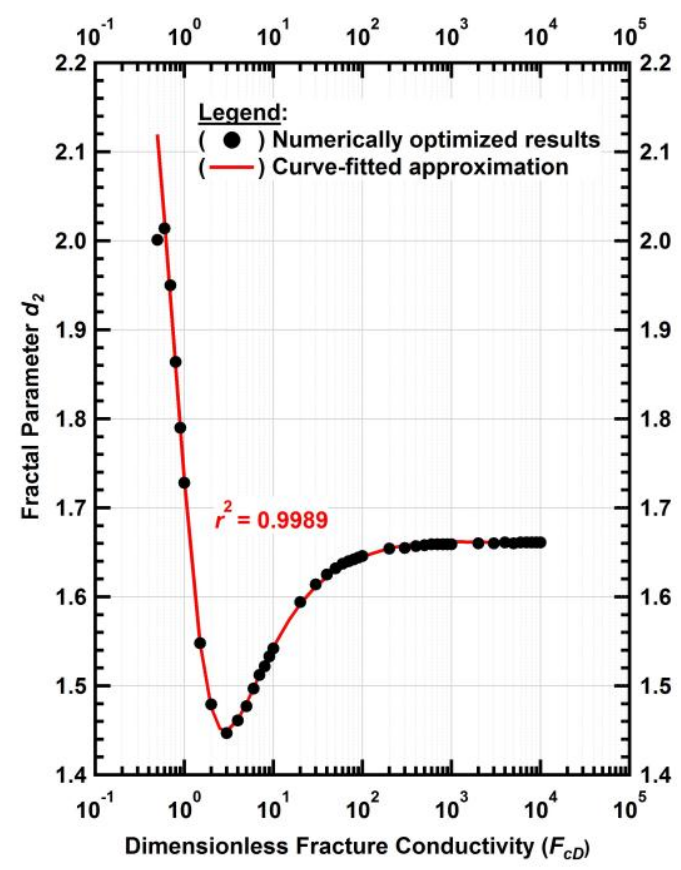

Figure 5.2 - Parameter $d_{2}$ versus dimensionless fracture conductivity $F_{c D}$. Each black circle represents the optimized $d_{2}$ for a given $F_{c D}$ value. The red line is the model approximation. The parameter $d_{2}$ is correlated solely in terms of $F_{c D}$. 
- $d_{3}=f\left(d_{2}\right): d_{3}$ is a defined function of $d_{2}$

This correlation is defined based on the observed behavior of the parameters $d_{3}$ versus $d_{2}$ (see Figure

5.3). From Figure 5.3 we considered that a quadratic form would best fit this relationship.

$$
d_{3}=A_{3}\left(d_{2}\right)^{2}+B_{3} d_{2}+C_{3}
$$

The following coefficients were determined using the TableCurve 2D software (Systat 2012):

$$
\begin{array}{lll}
A_{3}=1.4814 & B_{3}=-7.3109 & C_{3}=10.03
\end{array}
$$

- $\theta_{3}=f\left(d_{3}\right): \quad \theta_{3}$ is a defined function of $d_{3}$

This correlation is defined based on the observed behavior of the parameters $\theta_{3}$ versus $d_{3}$ (see Figure

5.4). From Figure 5.4 we considered that a linear form would best fit this relationship.

$$
\theta_{3}=A_{4} d_{3}+B_{4}
$$

The following coefficients were determined using the TableCurve 2D software (Systat 2012):

$$
A_{4}=1.2063 \quad B_{4}=-3.1532
$$

We next utilize our correlation relations (i.e., Eqs. 5.1-5.4) as components of the Fractal-Fracture Solution (FFS) procedure (i.e., Eq. 4.34 for Scenario 2). Using this FFS for Scenario 2, we then generate comparator cases ( $p_{D}$ and $p_{D}{ }^{\prime}$ functions) to visually assess the relative accuracy of our approach for this scenario. As all of the parametric correlations have very good to excellent statistical behavior, our expectation is that this FFS formulation should yield good correlations with the reference solution (CincoMeng). We expect that we may observe minor discrepancies in the FFS formulation for $F_{c D}<3$ due to the (relatively) irregular behavior of the correlations for the $\theta_{2}$ and $d_{2}$ parameters in the region of $F_{c D}<3$. 


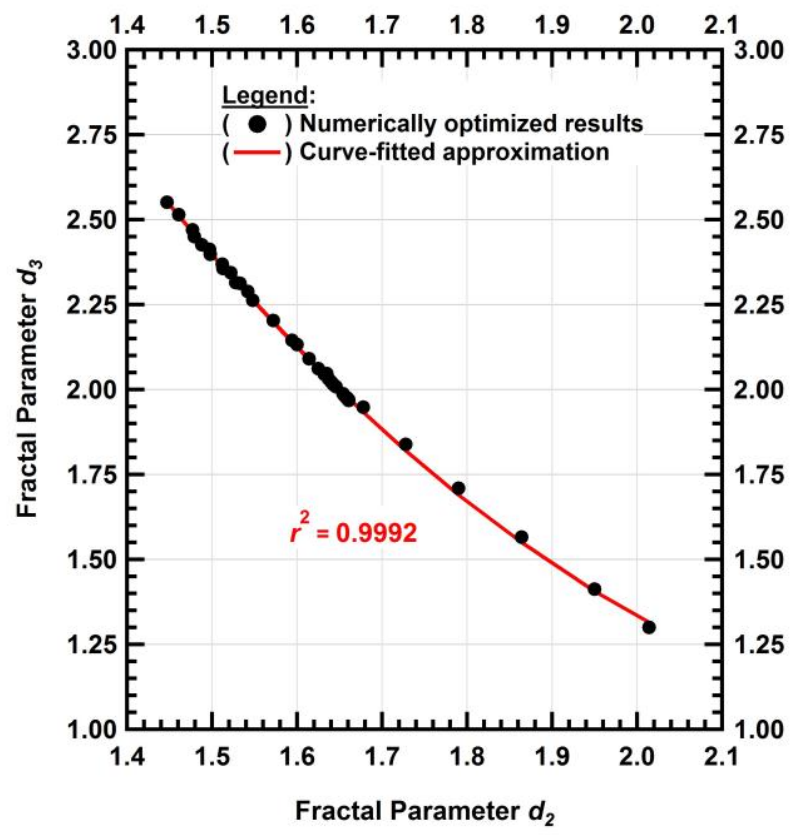

Figure 5.3 - Parameter $d_{3}$ versus parameter $d_{2}$. Each black circle represents the optimized $d_{3}$ value compared to a corresponding optimized $d_{2}$ value. The red line is the model approximation. In this rendering, the parameter $d_{3}$ is correlated solely in terms of the parameter $d_{2}$ using a quadratic trend — which seems appropriate given the trending of these data.

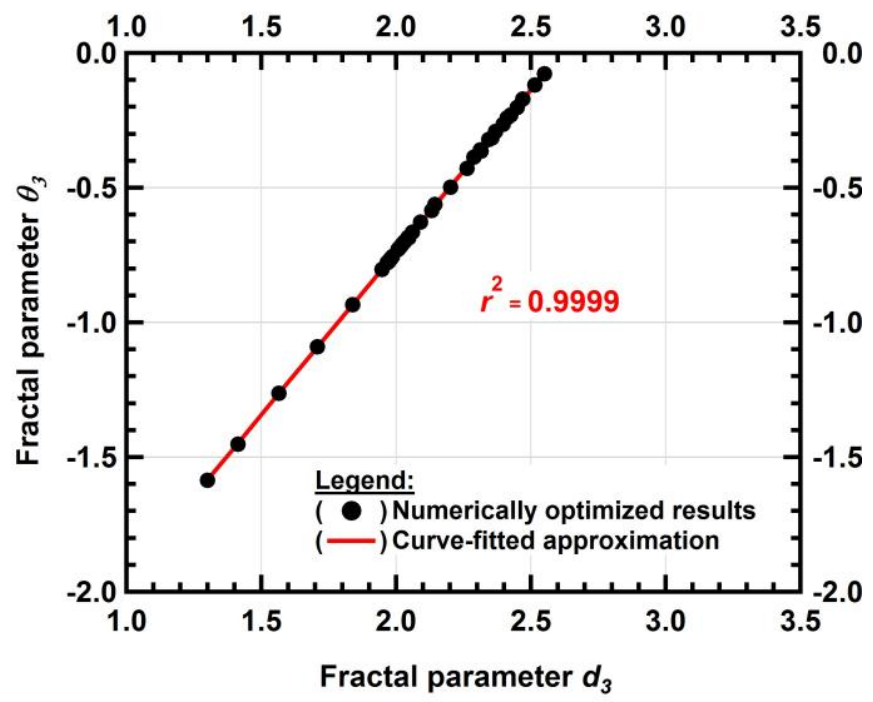

Figure 5.4 - Parameter $\theta_{3}$ versus parameter $d_{3}$. Each black circle represents the optimized $\theta_{3}$ value compared to a corresponding optimized $d_{3}$ value. The red line is the model approximation. . In this rendering, the parameter $\theta_{3}$ is correlated solely in terms of the parameter $d_{3}$ using a linear trend - which seems appropriate given the trending of these data. 


\subsection{Scenario 2: Error Analysis}

We now present results generated using the "Scenario 2" closed-form Fractal-Fracture Solution (i.e., Eqs. 4.34, 5.1-5.4) compared to the "reference" solution of Cinco-Meng (1988). In this comparison we vary the dimensionless fracture conductivity $\left(F_{c D}\right)$ over the range of $0.6 \leq F_{c D} \leq 10^{4}$, where this range encompasses very low conductivity $(0.6)$ to near-infinite conductivity $\left(10^{4}\right)$. Our approach is to compare the dimensionless pressure function $\left(p_{D}\right)$ and dimensionless pressure derivative function $\left(p_{\mathrm{D}}{ }^{\prime}\right)$ separately in order to assess the relative accuracy of each function in isolation.

Dimensionless Pressure: $p_{\underline{D}}$ versus $t_{\underline{D}}(\log$ - $\log$ format $)$ for $0.6 \leq F_{\underline{c} \underline{D}} \leq 10^{4}$

In Figure 5.5 we present the $p_{D}$ versus $t_{D}$ functions for FFS (Scenario 2) and the Cinco-Meng reference solution in $\log$ - $\log$ format $\left(13 \log\right.$ cycles are shown, this is 13 orders of magnitude in $\left.t_{D}\right)$. There appears to be only minor discrepancies at very small values of $t_{D}$ for the low conductivity cases $\left(0.6 \leq F_{c D} \leq 1\right)$. In this "log-log" view, there are no other apparent discrepancies in the solutions.

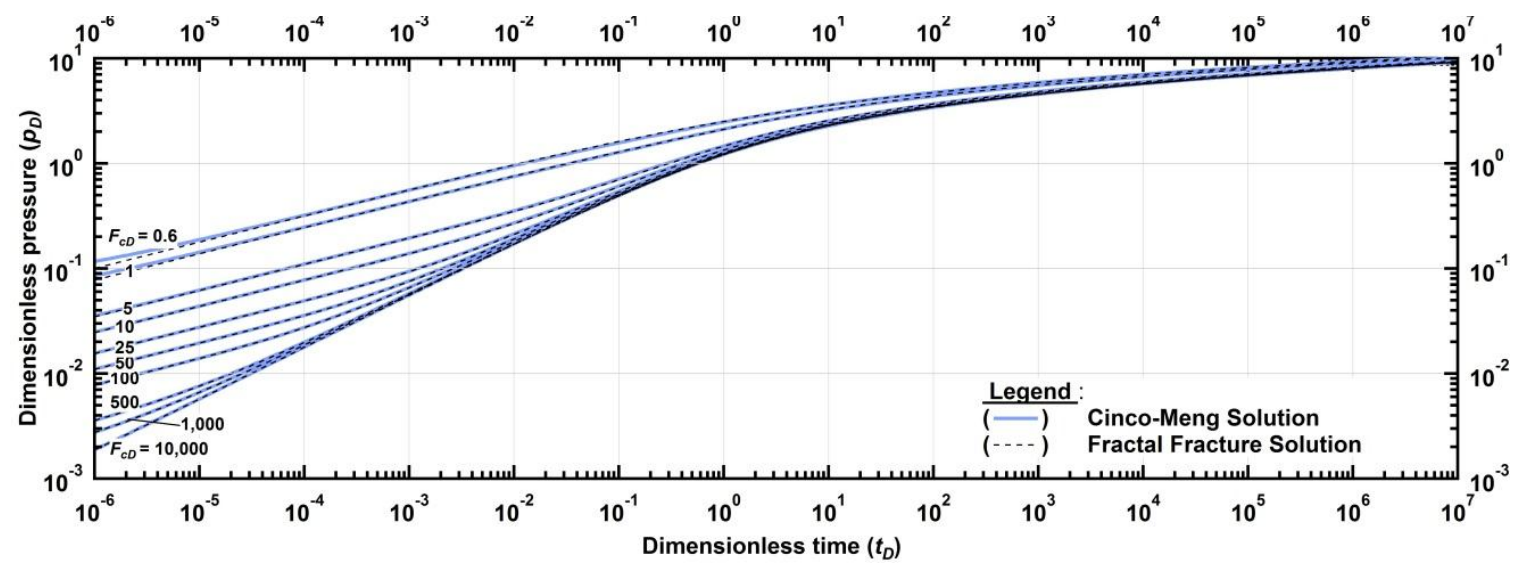

Figure 5.5 - (Scenario 2) Log-log plot of dimensionless pressure function versus dimensionless time for the FFS and Cinco-Meng Solutions (1988). 
Dimensionless Pressure: $p_{\underline{D}}$ versus $t_{\underline{D}}$ (semi-log $\left(x\right.$-axis) format) for $0.6 \leq F_{\underline{C} D} \leq 10^{4}$

In Figure 5.6 we present the $p_{D}$ versus $t_{D}$ functions for FFS (Scenario 2) and the Cinco-Meng reference solution in semi-log (x-axis) format (13 log cycles in $t_{D}$ are shown). There are no obvious discrepancies in this "semi-log" view, the correlation of the FFS and Cinco-Meng solutions appears to be excellent. As an effort to distinguish error at all scales, we next provide a relative error function presented against $t_{D}$ (in this work we use the "relative error" and " $\mathrm{L}^{1}$ error norm" functions expressed in percent).

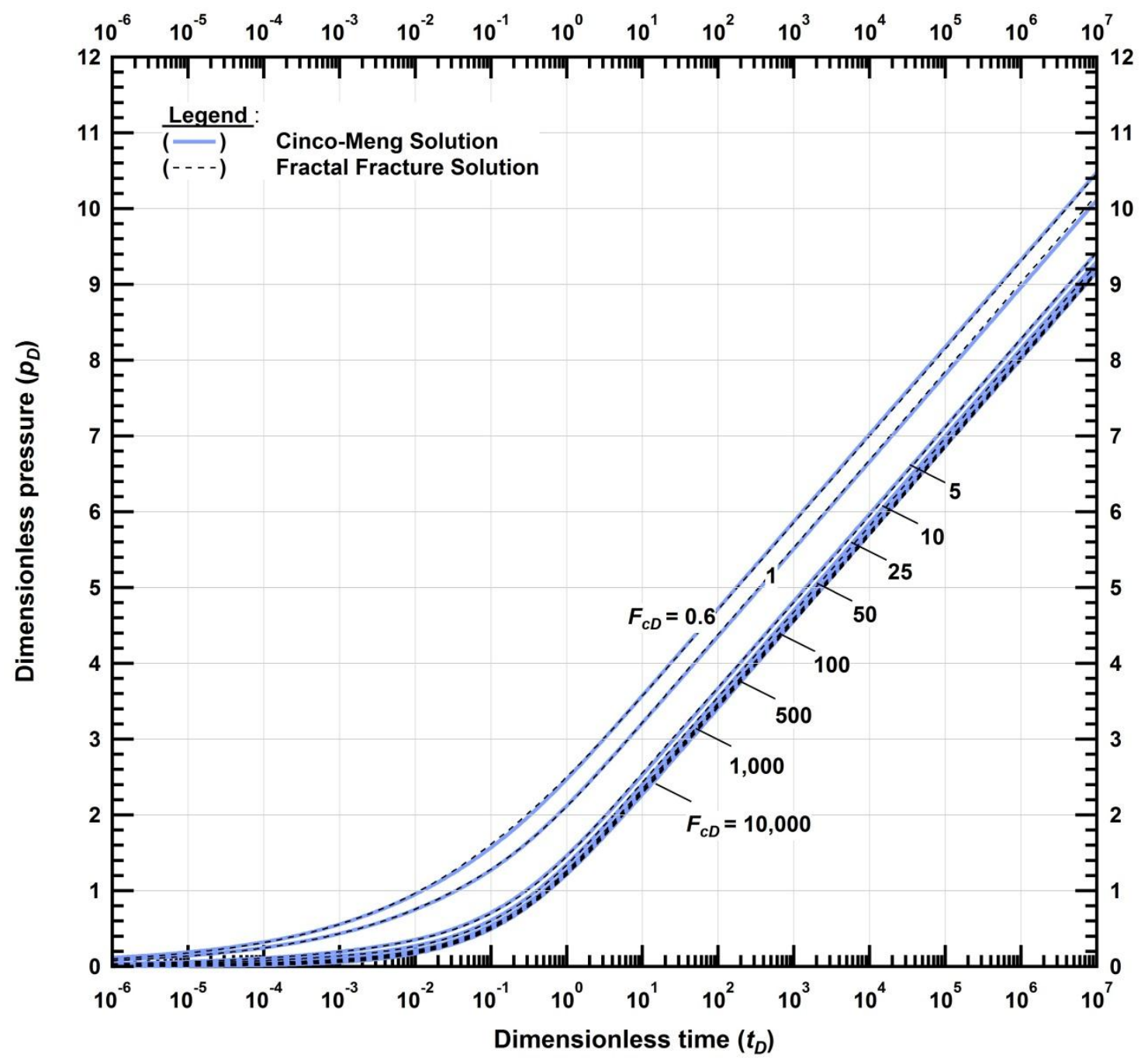

Figure 5.6 - (Scenario 2) Semi-log plot of dimensionless pressure versus dimensionless time for the FFS and Cinco-Meng (1988) Solutions. 
Dimensionless Pressure: $p_{\underline{D}}$ relative error versus $t_{\underline{D}}(\operatorname{semi}-\log (x$-axis $))$

In Figure 5.7 we present the relative error for the $p_{D}$ function (for $0.5 \leq F_{c D} \leq 10^{2}$ ) - where the definition of the relative error is given by:

Relative Error $=100\left[\left(p_{D, F F S}-p_{D, C M S}\right) / p_{D, C M S}\right]$ (percent)

Where $p_{D, F F S}$ is the FFS defined in this work, and the $p_{D, C M S}$ is the Cinco-Meng Solution (1988) (our reference for this case). The oscillatory nature of the FFS is evident in Figure 5.7, with the most dramatic oscillations occurring for the cases of $0.5 \leq F_{c D} \leq 1$. At very early times, the accuracy is very sensitive to $F_{c D}$ - for example, for cases where $F_{c D}<2.5$ the error becomes unacceptably high (above 4 percent at $\left.t_{D}=10^{-6}\right)$. At later times, the error corresponding to all fracture conductivities is generally low, with the only exceptions being the $F_{c D}=0.5$ and 0.6 curves.

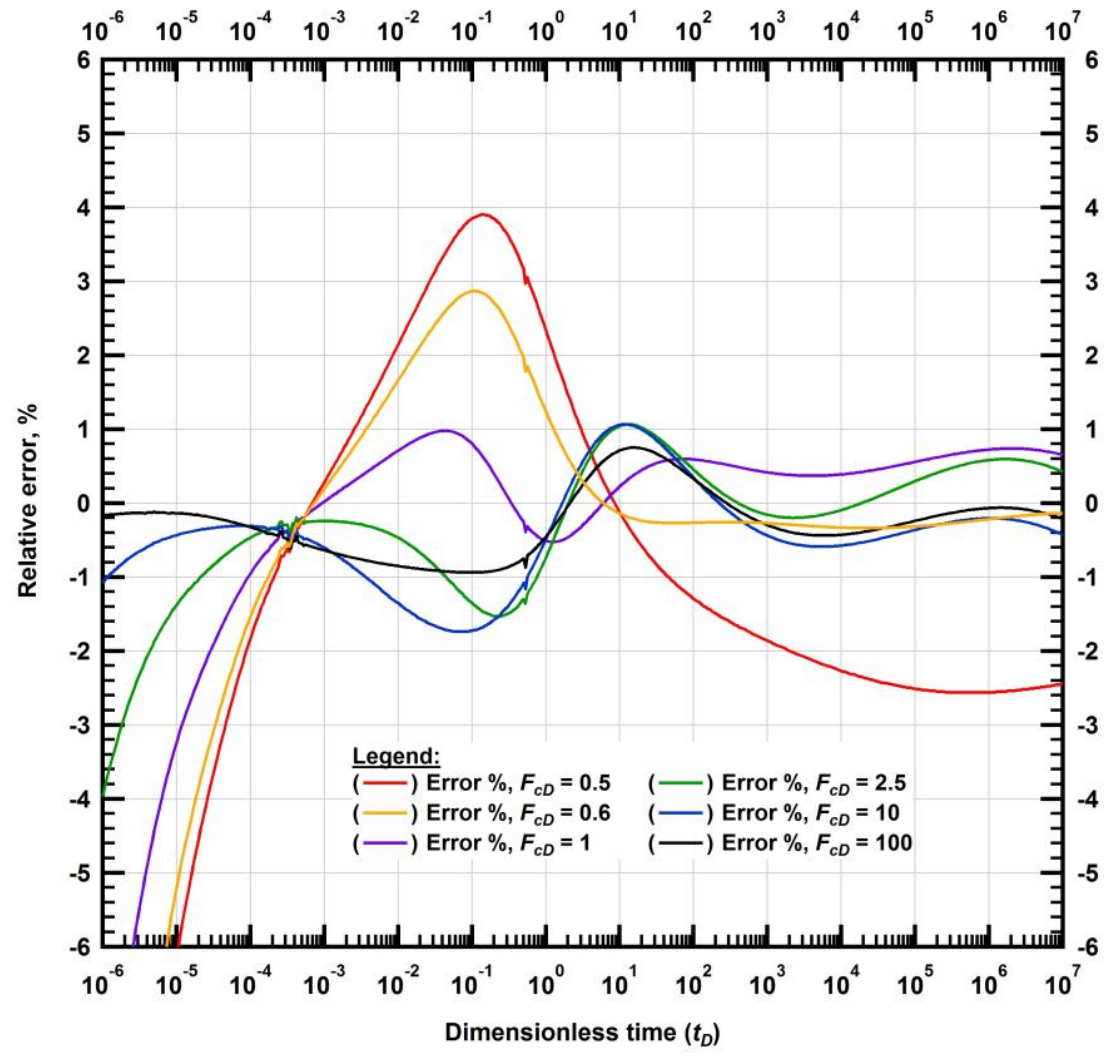

Figure 5.7 - (Scenario 2) Relative error (percent) in the dimensionless pressure solutions versus dimensionless time. 
Dimensionless Pressure: $p_{\underline{D}} \mathrm{~L}^{1}$ error norm versus $t_{\underline{D}}$ (semi-log $\left(x\right.$-axis)) for $0.6 \leq F_{\underline{c D}} \leq 10^{4}$

In Figure 5.8 we present the $\mathrm{L}^{1}$ error norm for the $p_{D}$ function (for $0.6 \leq F_{c D} \leq 10^{4}$ ) versus the dimensionless fracture conductivity $\left(F_{c D}\right)$, for the Fractal-Fracture Solution (FFS) and the Trilinear Pseudoradial Solution (TPRS) (Blasingame and Poe 1993). The definition of the $\mathrm{L}^{1}$ error norm is given by:

$\mathrm{L}^{1}$ error norm $=100\left[\left\|\boldsymbol{p}_{\boldsymbol{D}, F F S}-\boldsymbol{p}_{\boldsymbol{D}, C M S}\right\| /\left\|\boldsymbol{p}_{\boldsymbol{D}, \text { CMS }}\right\|\right]$ (percent)

Where $\boldsymbol{p}_{\boldsymbol{D}}$ is a vector containing values of $p_{D}$ for each specified $t_{D}$ value.

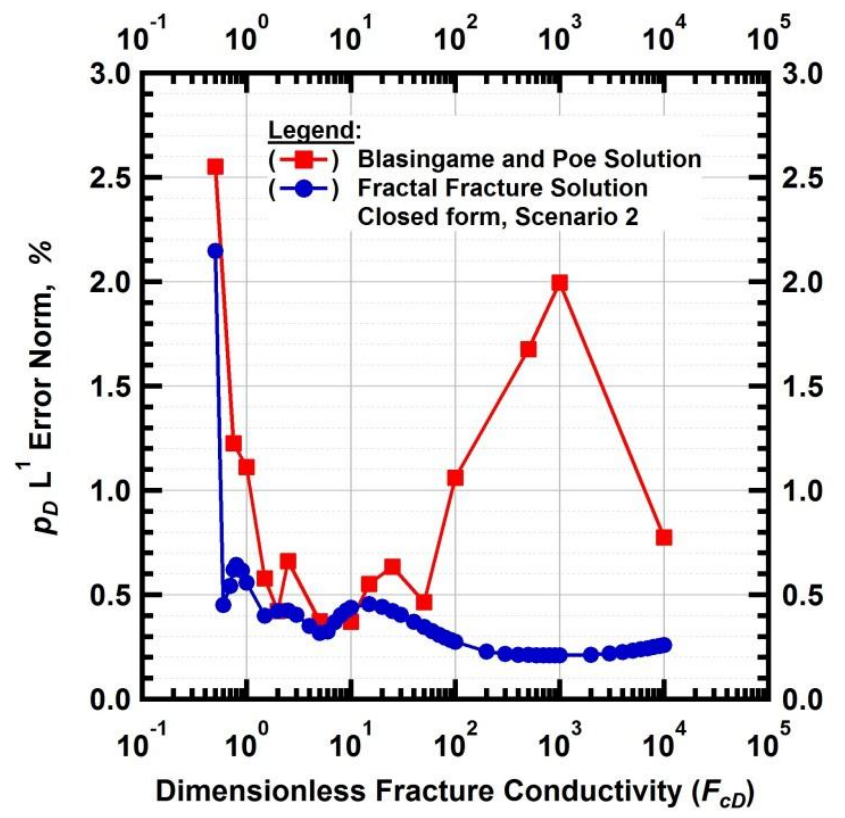

Figure 5.8 - (Scenario 2) $\mathrm{L}^{1}$ relative error norms for the dimensionless pressure solutions for the closed form Fractal-Fracture Solution (FFS) and the Trilinear Pseudoradial Solution (TPRS) (Blasingame and Poe 1993) versus the dimensionless fracture conductivity $\left(F_{c D}\right)$. Reference solution obtained from Cinco-Meng (1988). 
In Figure 5.8 we observe that the $p_{D}\left(t_{D}\right)$ values generated using the FFS are generally more accurate and more stable than obtained those from the Trilinear Pseudoradial Solution (TPRS) proposed by Blasingame and Poe (1993). We observe that the Blasingame and Poe (1993) solution (TPRS) exhibits its worst behavior in the $p_{D}$ function (i.e., $0.5 \leq \mathrm{L}^{1}$ error norm $\leq 2$ percent) for the range of $10^{2} \leq F_{c D} \leq 10^{4}$, which is actually somewhat unexpected since the TPRS uses the infinite-conductivity vertical fracture solution as its basis. Regardless, both the FFS and the TPRS methods should be more than sufficiently accurate for practical applications.

Dimensionless Pressure Derivative: $p_{\underline{D}}{ }^{\prime}$ versus $t_{\underline{D}}$ (log-log format) for $0.6 \leq F_{\underline{c} \underline{D}} \leq 10^{4}$

In Figure 5.9 we present the $p_{D}{ }^{\prime}$ versus $t_{D}$ functions for FFS (Scenario 2) and the Cinco-Meng reference solution in $\log -\log$ format $\left(13 \log\right.$ cycles are shown, this is 13 orders of magnitude in $\left.t_{D}\right)$. There appears to be only a very minor discrepancy at extremely small values of $t_{D}$ for the $F_{c D}=0.6$ conductivity case. In short, in this log-log view, we observe no significant issues/discrepancies in the solutions.

Dimensionless Pressure Derivative: $\underline{p}_{\underline{D}}{ }^{\prime}$ versus $t_{\underline{D}}$ (semi-log ( $x$-axis) format) for $0.6 \leq F_{\underline{c} \underline{D}} \leq 10^{4}$

In Figure 5.10 we present the $p_{D}{ }^{\prime}$ versus $t_{D}$ functions for FFS (Scenario 2) and the Cinco-Meng reference solution in semi-log $\left(x\right.$-axis) format $\left(13 \log\right.$ cycles in $t_{D}$ are shown). As opposed to the log-log view of these data (i.e., Figure 5.9) we observe significant discrepancies in the $p_{D}{ }^{\prime}$ versus $\log \left(t_{D}\right)$ presentation specifically, all cases exhibit significant oscillations in the $p_{D}{ }^{\prime}$ function for $t_{D} \geq 1$. Although somewhat speculative, we believe that the oscillations in the $p_{D}{ }^{\prime}$ function are due to the nature of the FFS - the fact that we used a fractal concept to represent a non-fractal process. While these oscillations are not trivial, we do believe that the FFS process yields a reasonable approximation for the $p_{D}{ }^{\prime}$ function; and most likely, this approach is sufficient for practical applications. 


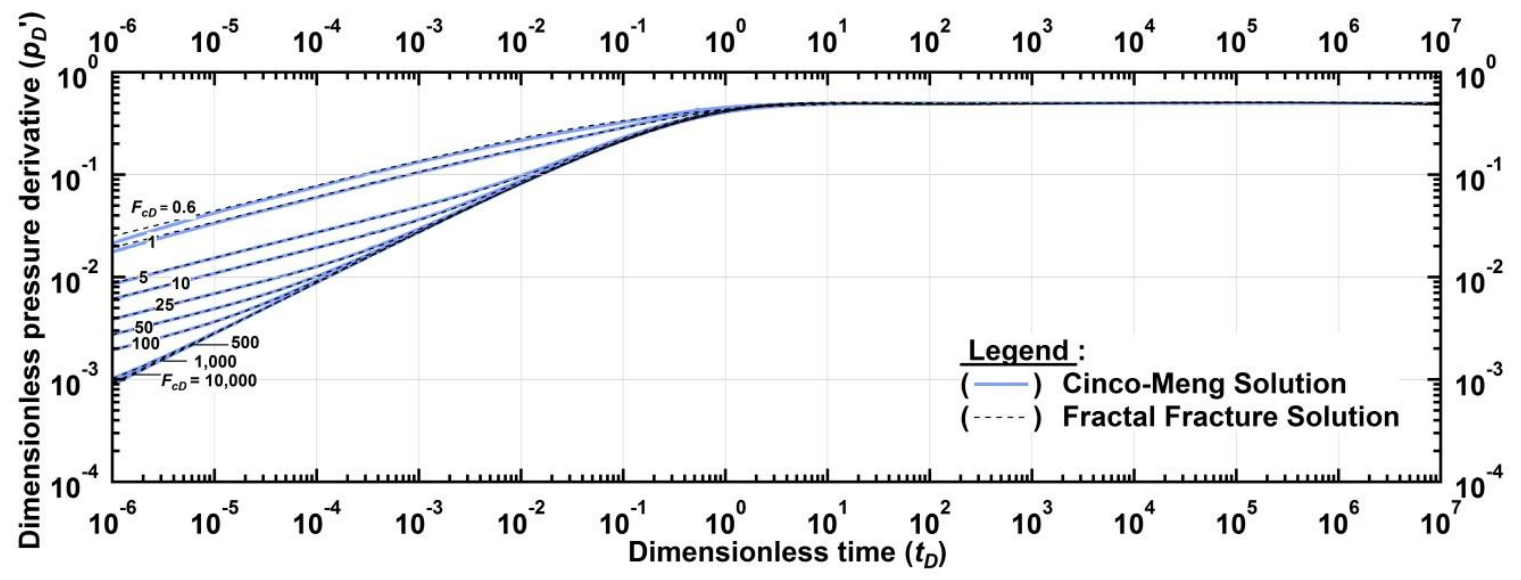

Figure 5.9- (Scenario 2) Log-log plot of dimensionless pressure derivative function versus dimensionless time for the FFS and Cinco-Meng Solutions (1988).

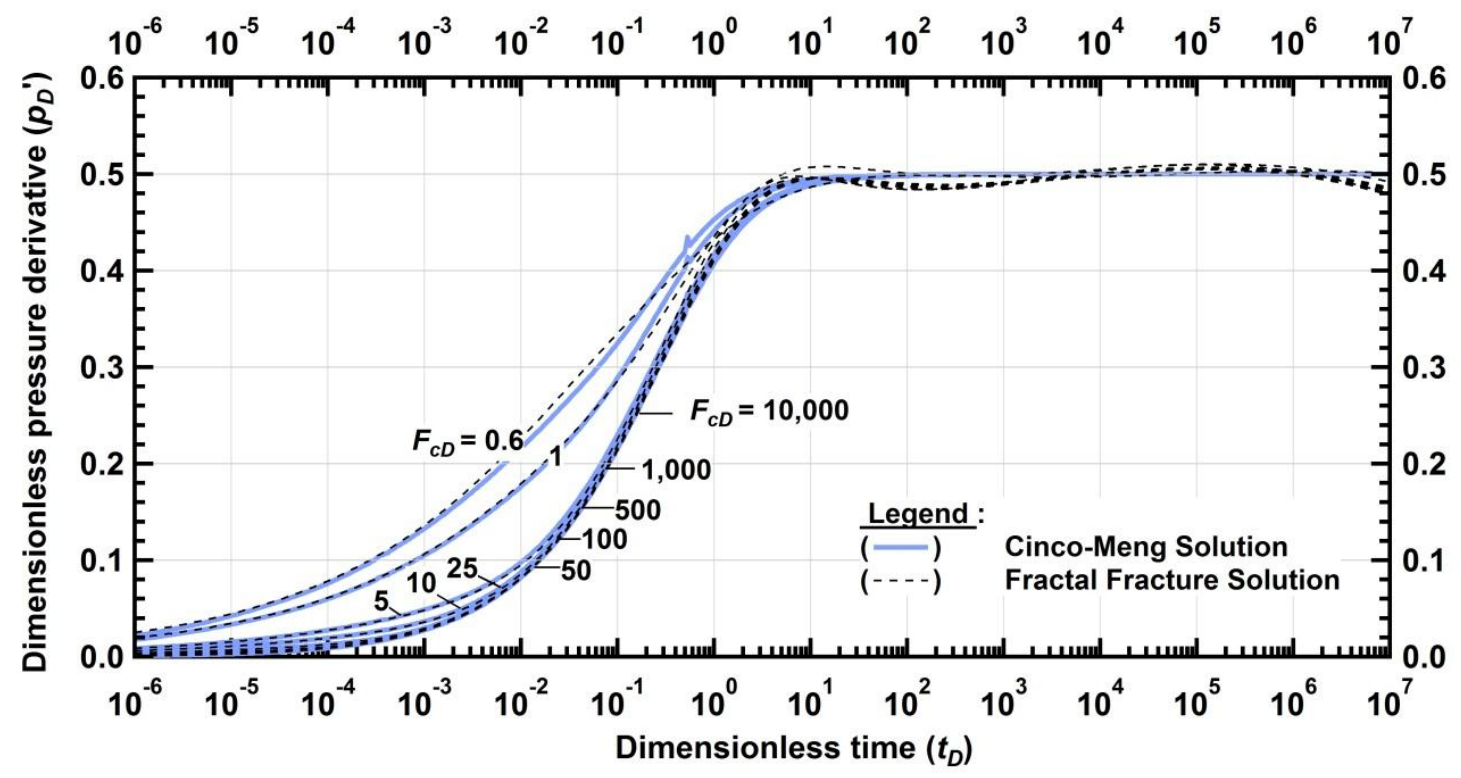

Figure 5.10 - (Scenario 2) Semi-log plot of dimensionless pressure derivative versus dimensionless time for the FFS and Cinco-Meng Solutions (1988). 
Dimensionless Pressure Derivative: $p_{\underline{D}}{ }^{\prime}$ relative error versus $t_{\underline{D}}$ (semi-log $(x$-axis $\left.)\right)$

The relative error for the $p_{D}{ }^{\prime}$ function is presented in Figure 5.11 (for $0.5 \leq F_{c D} \leq 10^{2}$ ) - and our first observation (as might be expected since this is a derivative function) is that the relative error for the $p_{D}{ }^{\prime}$ function is more "oscillatory" than that of the $p_{D}$ function — both in amplitude and frequency. The range of cases considered is the same as for the $p_{D}$ function case $\left(\right.$ i.e., $0.5<F_{c D}<10^{2}$ ) and we note the greatest magnitudes of errors for the $F_{c D}=0.5$ and 0.6 curves (which was also the case for the $p_{D}$ function). We observe that all cases have a maximum relative error of at least 3 percent - and the $F_{c D}=0.5$ case has two error peaks over 6 percent, as well as errors over 10 percent for very small values of $t_{D}$. As comment, this behavior is somewhat expected in a derivative function, as the visual oscillations in the FFS confirm in Figure 5.10.

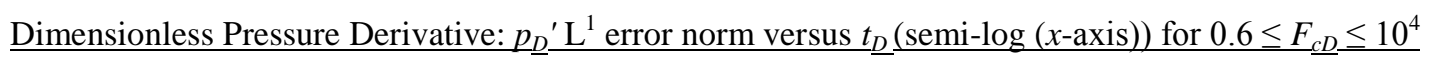

In Figure 5.12 we present the $\mathrm{L}^{1}$ error norm for the $p_{D}{ }^{\prime}$ function (for $0.6 \leq F_{c D} \leq 10^{4}$ ) versus the dimensionless fracture conductivity $\left(F_{c D}\right)$, for the Fractal-Fracture Solution (FFS) and the Trilinear Pseudoradial Solution (TPRS) (Blasingame and Poe 1993). As would be expected from the comparison of relative errors in Figure 5.11, $\mathrm{L}^{1}$ error norms for the FFS are highest for $0.6 \leq F_{c D} \leq 2$ (ranging between 1 and 2 percent). In contrast, the Blasingame and Poe (1993) solution (TPRS) exhibits relatively stable behavior in the $\mathrm{L}^{1}$ error norm — in particular $0.7 \leq \mathrm{L}^{1}$ error norm $\leq 1.2$ percent, and varies somewhat randomly with $F_{c D}$, although the highest observed errors occur for the $10^{2} \leq F_{c D} \leq 10^{4}$ cases.

As mentioned in the error analysis for the $p_{D}$ functions, we believe that the FFS and TPRS methods are sufficiently accurate for practical applications, but the observed oscillatory behavior of the $p_{D}{ }^{\prime}$ functions as shown in Figure 5.10 confirms that the FFS method can (and should) be improved. Any improvements should reduce the oscillatory nature of the $p_{D}{ }^{\prime}$ functions as well as reduce the relative errors and the $\mathrm{L}^{1}$ error norm behavior. 


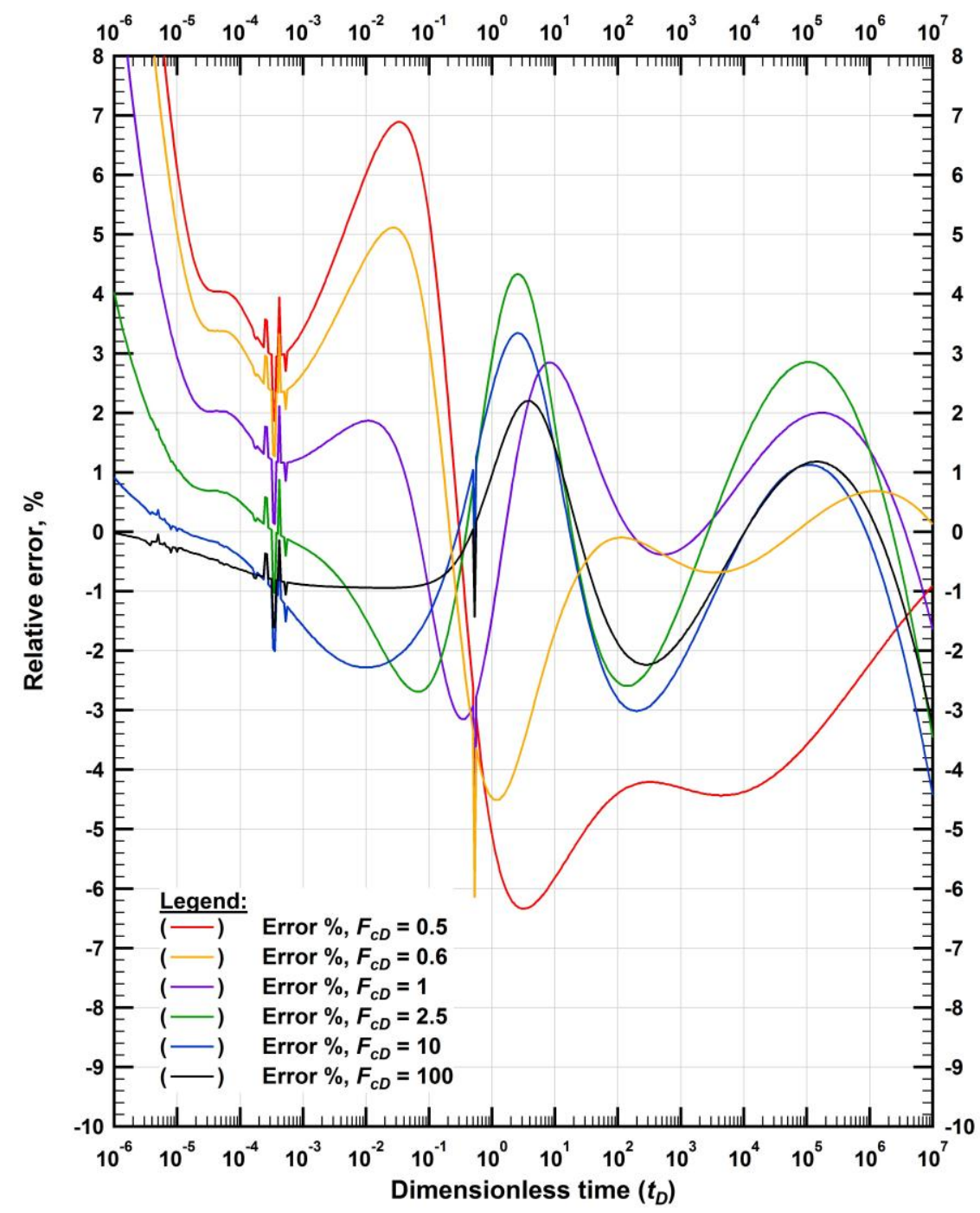

Figure 5.11 - (Scenario 2) Relative error (percent) in the dimensionless pressure derivative solutions versus dimensionless time. 


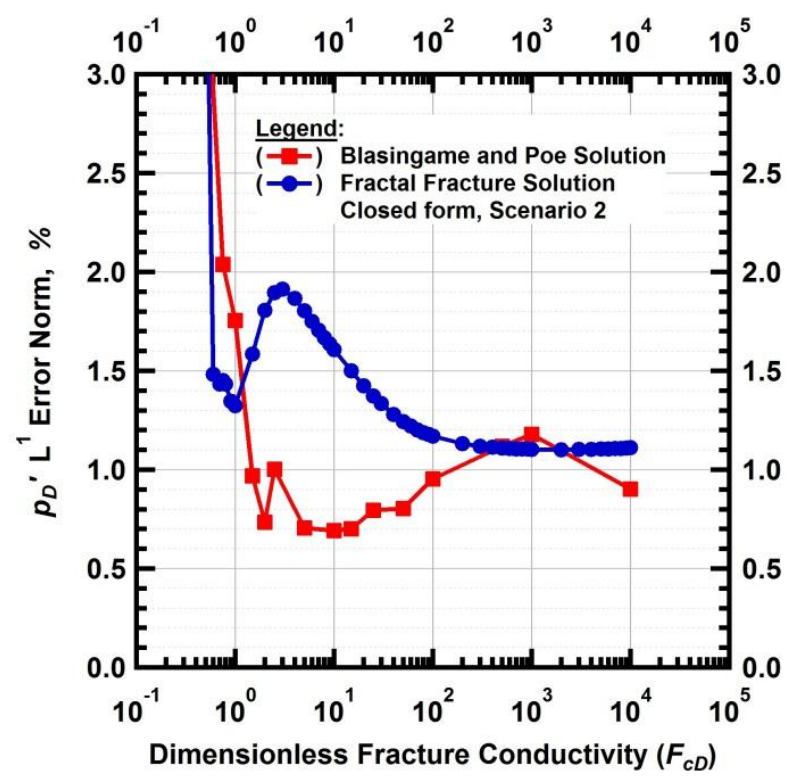

Figure 5.12 - (Scenario 2) $\mathrm{L}^{1}$ relative error norms for the dimensionless pressure derivative solutions for the closed form Fractal-Fracture Solution (FFS) and the Trilinear Pseudoradial Solution (TPRS) (Blasingame and Poe 1993) versus the dimensionless fracture conductivity $\left(F_{c D}\right)$. Reference solution obtained from Cinco-Meng (1988).

\subsection{Scenario 3: Parameter Correlations}

Our prior work with Scenario 2 [i.e., Region 1 (the fracture) non-fractal, Regions 2 and 3 fractal - Table 4.1] proved that we have room for improvement in terms of the performance of the Fractal-Fracture Solution (FFS) method - as such, we now pursue development of Scenario 3 [all Regions fractal Table 4.1]. In our development of Scenario 3 we will limit ourselves to the following conditions:

- Cases where $F_{c D} \geq 3$, as this appears to be the "tipping point" where the FFS method may fail.

- Parametric correlation relations (models) will contain no more than 10 coefficients..

For reference, the numerically optimized fractal parameters for both Scenarios 2 and 3 are shown in tabular fashion in Appendix A (these results form the basis for our correlations) — and, as a recommendation of this work, we invite the reader to create his/her own more sophisticated correlations [see also Figure $4.10\left(d_{1}, d_{2}, d_{3}\right)$ and Figure $\left.4.11\left(\theta_{1}, \theta_{2}, \theta_{3}\right)\right]$ for a graphical presentation of the optimized 
parameters. We note that a "perfect" correlation for all fractal parameters would match each point in Figure 4.10 and Figure 4.11.

As with the Scenario 2, we develop parameter-specific correlations — in particularly, for Scenario 3 we constructed the following correlations:

- $\theta_{1}=f\left(F_{c D}\right): \theta_{1}$ is a unique function of $F_{c D}$

- $d_{3}=f\left(F_{c D}\right): d_{3}$ is a unique function of $F_{c D}$

- $\theta_{3}=f\left(d_{3}\right): \quad \theta_{3}$ is a defined function of $d_{3}$

- $d_{1}=f\left(F_{c D}\right): d_{1}$ is a unique function of $F_{c D}$

- $\theta_{2}=f\left(d_{1}\right): \quad \theta_{2}$ is a defined function of $d_{1}$

- $d_{2}=f\left(d_{1}\right): d_{2}$ is a defined function of $d_{1}$

The parameter correlations for Scenario 3 are provided below:

- $\theta_{1}=f\left(F_{c D}\right): \theta_{1}$ is a unique function of $F_{c D}$

$$
\theta_{1}=A_{1}+B_{1} \sqrt{x} \ln (x)+C_{1}(\ln x)^{2}+D_{1} / x^{1.5}\left(\text { where } x=\log _{10}\left(F_{c D}\right)\right)
$$

The following coefficients were determined using the TableCurve 2D software (Systat 2012):

$$
\begin{aligned}
& A_{1}=-0.74232260 \quad C_{1}=-1.40767202 \\
& B_{1}=1.186334039 \quad D_{1}=0.425529601
\end{aligned}
$$

The data-model correlation for this case is shown in Figure 5.13.

- $d_{3}=f\left(F_{c D}\right): d_{3}$ is a unique function of $F_{c D}$

$$
d_{3}=\left(A_{2}+C_{2} x^{2}\right) /\left(1+B_{2} x^{2}\right)\left(\text { where } x=\log _{10}\left(F_{C D}\right)\right)
$$

The following coefficients were determined using the TableCurve 2D software (Systat 2012):

$$
\begin{aligned}
& A_{2}=1.306668018 \\
& B_{2}=3.121888858
\end{aligned} \quad C_{2}=5.810553378
$$

The data-model correlation for this case is shown in Figure 5.14. 


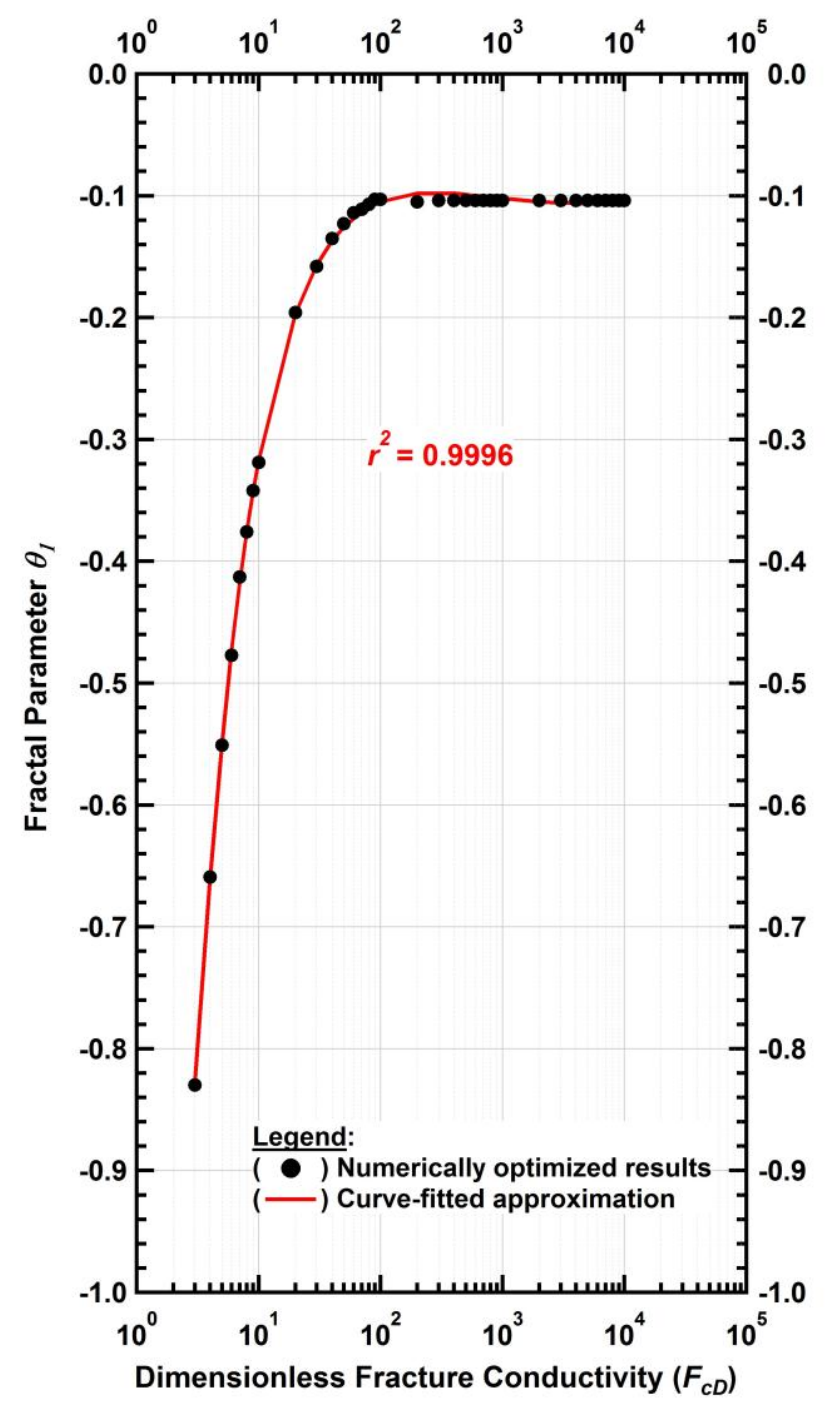

Figure 5.13 - Parameter $\theta_{1}$ versus dimensionless fracture conductivity $F_{c D}$. Each black circle represents the optimized $\theta_{1}$ for a given $F_{c D}$ value. The red line is the model approximation. The parameter $\theta_{1}$ is correlated solely in terms of $F_{c D}$. 


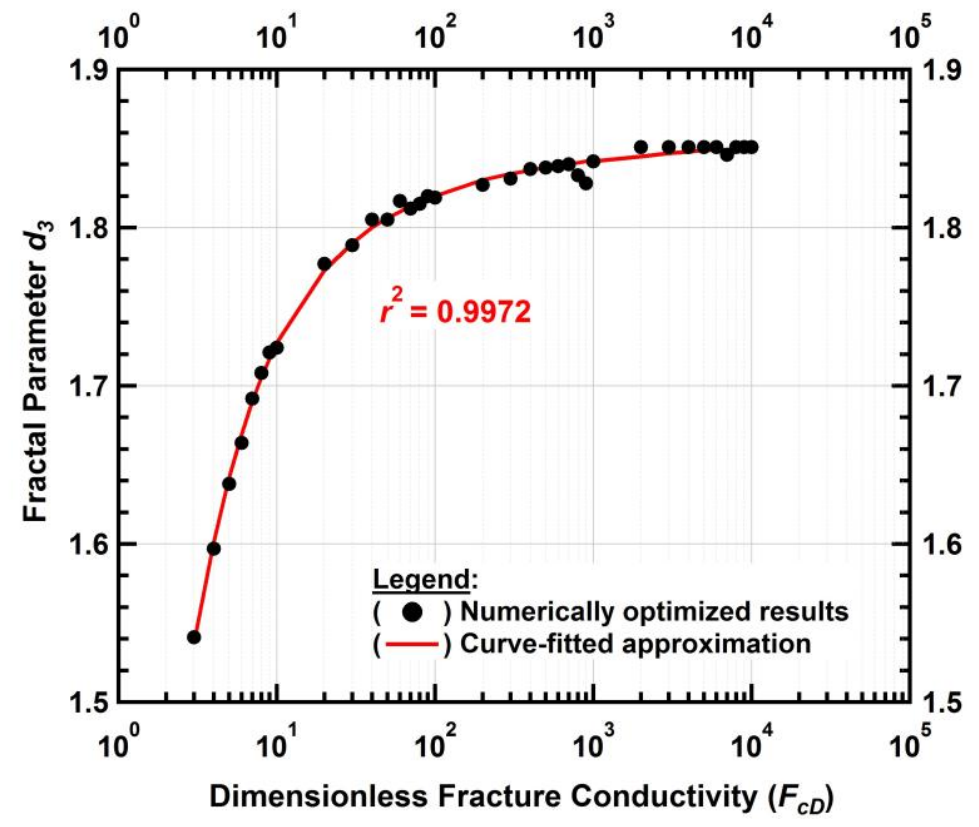

Figure 5.14 - Parameter $d_{3}$ versus dimensionless fracture conductivity $F_{c D}$. Each black circle represents the optimized $d_{3}$ for a given $F_{c D}$ value. The blue line is the model approximation. The parameter $d_{3}$ is correlated solely in terms of $F_{c D}$.

- $\theta_{3}=f\left(d_{3}\right): \theta_{3}$ is a defined function of $d_{3}$

$$
\theta_{3}=A_{3} d_{3}+B_{3}
$$

The following coefficients were determined using the TableCurve 2D software (Systat 2012):

$$
A_{3}=1.094 \quad B_{3}=-2.9493
$$

The data-model correlation for this case is shown in Figure 5.15. 


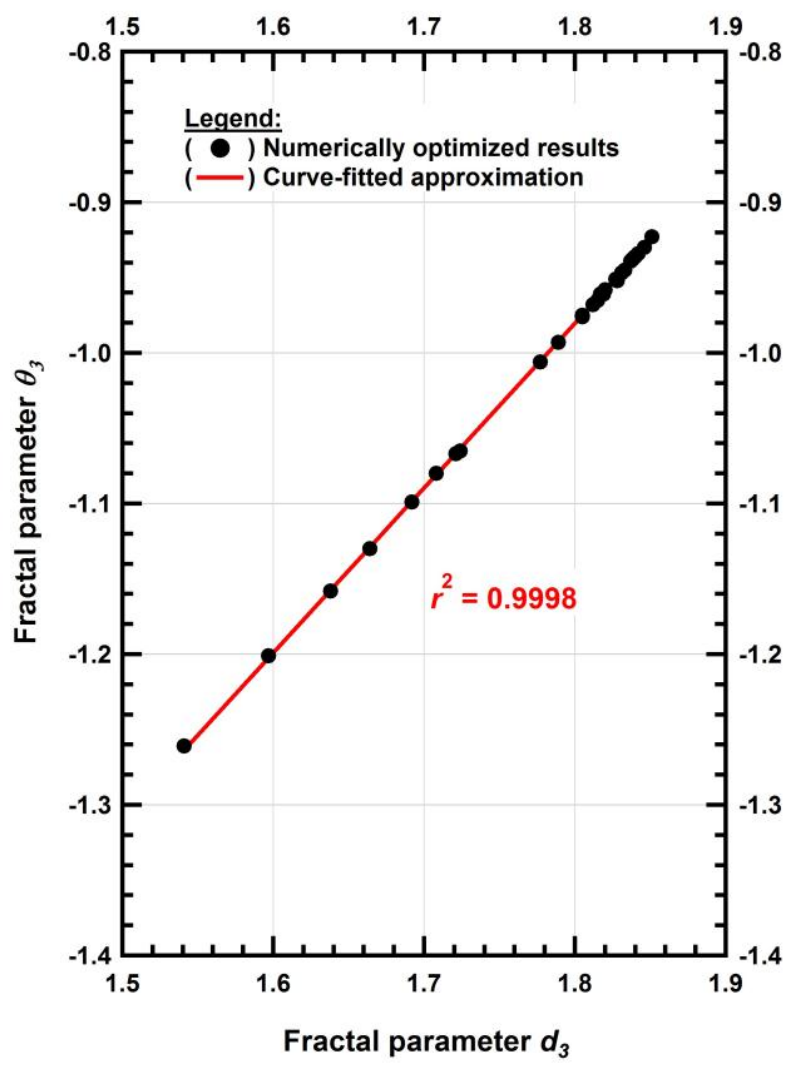

Figure 5.15 - Parameter $\theta_{3}$ versus parameter $d_{3}$. Each black circle represents the optimized $\theta_{3}$ for a given $d_{3}$ value. The red line is the model approximation. The parameter $\theta_{3}$ is correlated solely in terms of $d_{3}$.

- $d_{1}=f\left(F_{c D}\right): d_{1}$ is a unique function of $F_{c D}$

$$
d_{1}=A_{4}\left[1-\exp \left(-\frac{x-C_{4} \ln \left(1-\frac{\sqrt{2}}{2}\right)-B_{4}}{C_{4}}\right)\right]^{2}\left(\text { where } x=\log _{10}\left(F_{c D}\right)\right)
$$

The following coefficients were determined using the TableCurve 2D software (Systat 2012):

$$
\begin{aligned}
& A_{4}=1.977809038 \\
& B_{4}=0.045173200
\end{aligned} \quad C_{4}=0.472579200
$$

The data-model correlation for this case is shown in Figure 5.16.

- $\theta_{2}=f\left(d_{1}\right): \quad \theta_{2}$ is a defined function of $d_{1}$ 


$$
\theta_{2}=A_{5}\left(d_{1}\right)^{2}+B_{5} d_{1}+C_{5}
$$

The following coefficients were determined using the TableCurve 2D software (Systat 2012):

$$
\begin{array}{ll}
A_{5}=-0.5789 & C_{5}=3.2214 \\
B_{5}=2.6028 &
\end{array}
$$

The data-model correlation for this case is shown in Figure 5.17.

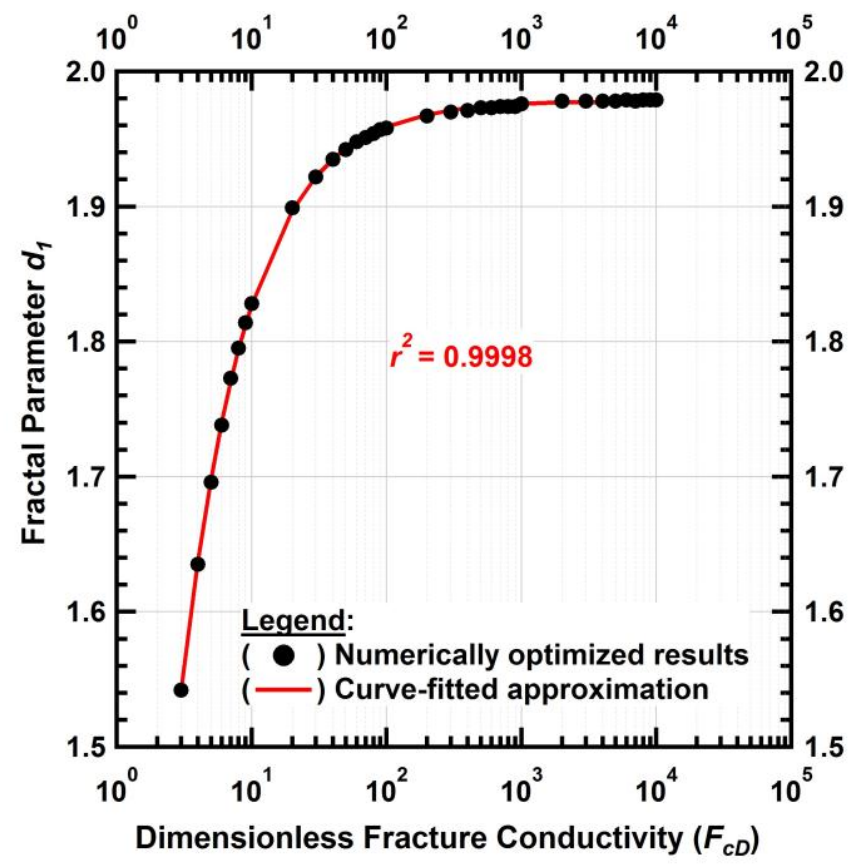

Figure 5.16 - Parameter $d_{1}$ versus dimensionless fracture conductivity $F_{c D}$. Each black circle represents the optimized $d_{1}$ for a given $F_{c D}$ value. The red line is the model approximation. The parameter $d_{1}$ is correlated solely in terms of $F_{c D}$. 


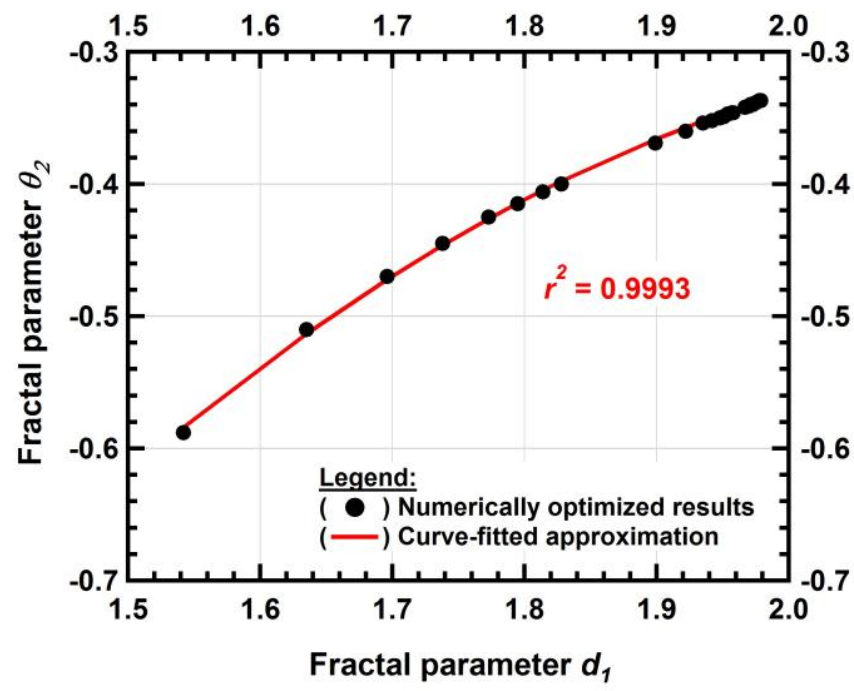

Figure 5.17 - Parameter $\theta_{2}$ versus parameter $d_{1}$. Each black circle represents the optimized $\theta_{2}$ for a given $d_{1}$ value. The red line is the model approximation. The parameter $\theta_{2}$ is correlated solely in terms of $d_{1}$.

- $d_{2}=f\left(d_{1}\right): d_{2}$ is a defined function of $d_{1}$

$$
d_{2}=A_{6}\left(d_{1}\right)^{2}+B_{6} d_{1}+C_{6}
$$

The following coefficients were determined using the TableCurve 2D software (Systat 2012):

$$
\begin{aligned}
& A_{6}=-0.4481 \\
& B_{6}=1.0935
\end{aligned} \quad C_{6}=1.3073
$$

The data-model correlation for this case is shown in Figure 5.18. 


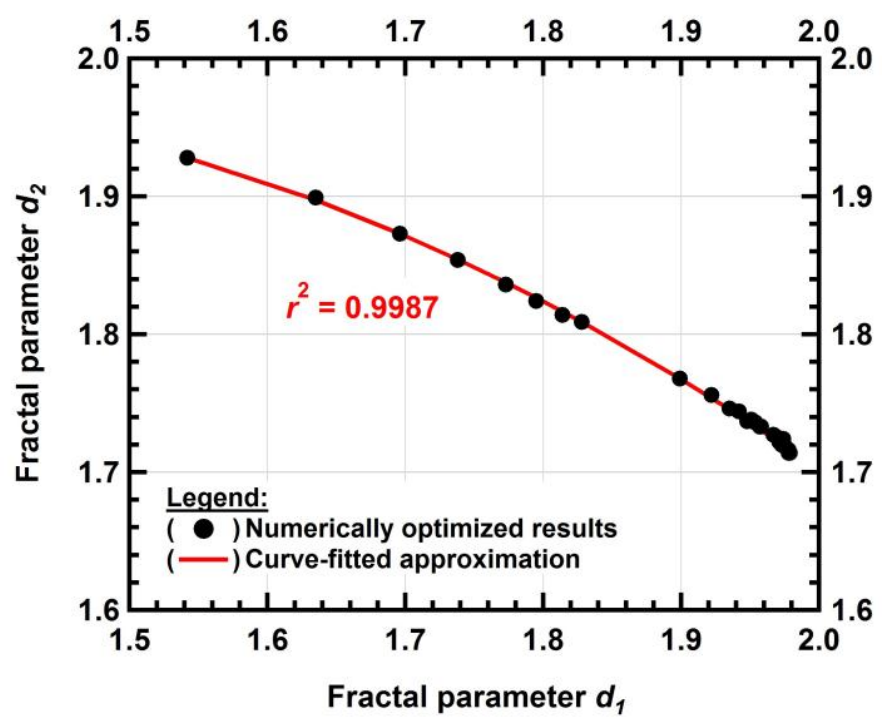

Figure 5.18 - Parameter $d_{2}$ versus parameter $d_{1}$. Each black circle represents the optimized $d_{2}$ for a given $d_{1}$ value. The red line is the model approximation. The parameter $d_{2}$ is correlated solely in terms of $d_{1}$.

As with Scenario 2, now that we have established correlations for all parameters in Scenario 3 (i.e., $d_{1}, d_{2}$, $d_{3}, \theta_{1}, \theta_{2}, \theta_{3}$ ), we proceed to generate comparator cases - in particular, we use the $p_{D}$ and $p_{D}{ }^{\prime}$ functions and associated relative error and $\mathrm{L}^{1}$ error norm cases to assess the relative accuracy of the FFS approach for Scenario 3. As with Scenario 2, all of our parametric correlations have very good to excellent statistical behavior, and our expectation is that this FFS formulation should yield very good results when compared with the reference solution (Cinco-Meng). For Scenario 3, only cases of $F_{c D} \geq 3$ are considered. 


\subsection{Scenario 3: Error Analysis}

We now present results generated using the "Scenario 3" closed-form Fractal-Fracture Solution (i.e., Eqs. 4.35, 5.7-5.12) compared to the "reference" solution of Cinco-Meng (1988). In this comparison we vary the dimensionless fracture conductivity $\left(F_{c D}\right)$ over the range of $3 \leq F_{c D} \leq 10^{4}$, where this range encompasses moderately low conductivity (3) to near-infinite conductivity $\left(10^{4}\right)$.

Dimensionless Pressure: $p_{\underline{D}}$ versus $t_{\underline{D}}(\log -\log$ format $)$ for $3 \leq F_{c D} \leq 10^{4}$

In Figure 5.19 we present the $p_{D}$ versus $t_{D}$ functions for FFS (Scenario 3) and the Cinco-Meng reference solution in log-log format ( $13 \log$ cycles are shown, this is 13 orders of magnitude in $\left.t_{D}\right)$. In this view the correlation is very strong between the FFS and Cinco-Meng (1998) reference cases — in short, there appear to be no discrepancies for any value $p_{D}\left(t_{D}\right)$ values.

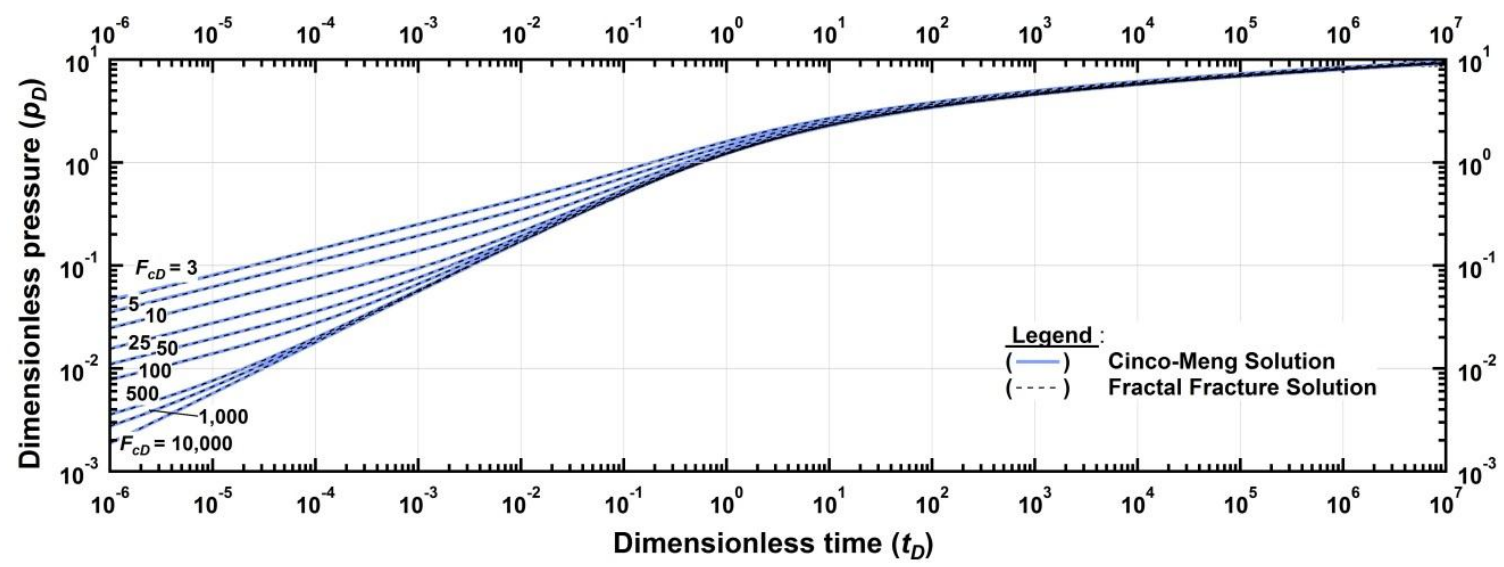

Figure 5.19 - (Scenario 3) Log-log plot of dimensionless pressure function versus dimensionless time for the FFS and Cinco-Meng Solutions (1988).

Dimensionless Pressure: $p_{\underline{D}}$ versus $t_{\underline{D}}$ (semi-log $\left(x\right.$-axis) format) for $3 \leq F_{\underline{c} \underline{ }} \leq 10^{4}$

In Figure 5.20 we present the $p_{D}$ versus $t_{D}$ functions for FFS (Scenario 3) and the Cinco-Meng reference solution in semi-log $\left(x\right.$-axis) format $\left(13 \log\right.$ cycles in $t_{D}$ are shown). We note an apparently perfect correlation of the FFS and Cinco-Meng solutions in this view. 


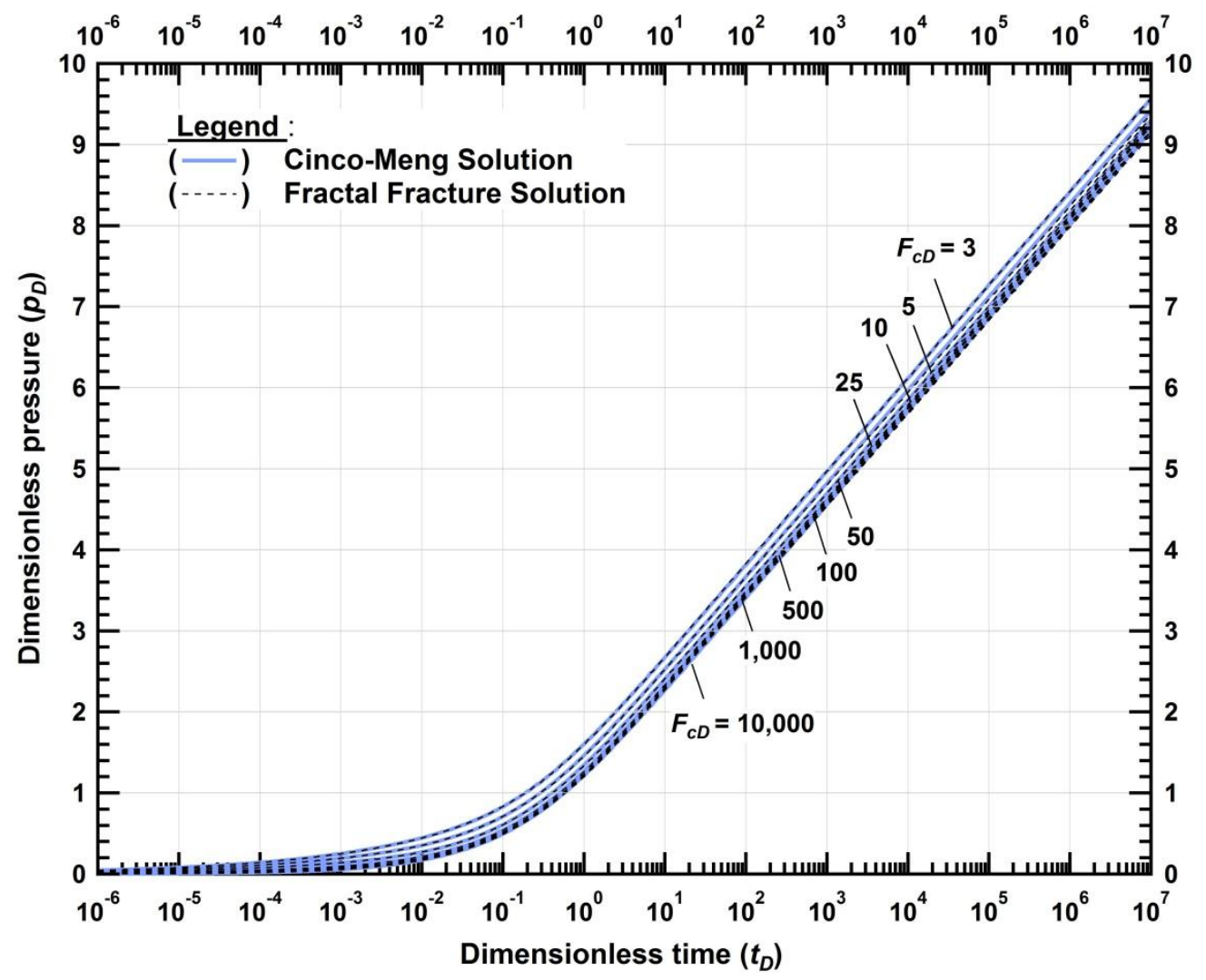

Figure 5.20 - (Scenario 3) Semi-log plot of dimensionless pressure versus dimensionless time for the FFS and Cinco-Meng Solutions (1988).

\section{Dimensionless Pressure: $\underline{p}_{\underline{D}}$ relative error versus $t_{D} \underline{(\text { semi- } \log (x \text {-axis }))}$}

In Figure 5.21 we present the relative error for the $p_{D}$ function (for $3 \leq F_{c D} \leq 10^{3}$ ) - and as with Scenario 2, the results for Scenario 3 do exhibit oscillatory behavior, but the limits on these errors are -0.5 to 0.5 percent (except at very early times). This suggests that Scenario 3 yields substantially more accuracy than Scenario 2 (recall that the relative error ranges for Scenario 2 were approximately -4 to 4 percent). The oscillatory behavior may be an issue which affects the $p_{D}{ }^{\prime}$ function (this will be discussed in the next section). 
Dimensionless Pressure: $p_{\underline{D}} \underline{L}^{1}$ error norm versus $t_{\underline{D}}\left(\right.$ semi-log $(x$-axis) $)$ for $3 \leq F_{\underline{c} \underline{D}} \leq 10^{3}$

In Figure 5.22 we present the $\mathrm{L}^{1}$ error norm for the $p_{D}$ function (for $3 \leq F_{c D} \leq 10^{3}$ ) versus the dimensionless fracture conductivity $\left(F_{c D}\right)$, for the Fractal-Fracture Solution (FFS) and the Trilinear Pseudoradial Solution (TPRS) (Blasingame and Poe 1993). The FFS and TPRS L ${ }^{1}$ error norms are essentially identical for $3 \leq F_{c D} \leq 10^{2}$, and the TPRS $\mathrm{L}^{1}$ error norm does drift up to almost 2 percent for $10^{2} \leq F_{c D} \leq 10^{4}$ while the FFS for this case has a range of 0.2 to 0.5 percent, suggesting that the FFS is a somewhat better approximation over the $10^{2} \leq F_{c D} \leq 10^{4}$ range. It is relevant to note that we have limited the FFS solution to cases where $F_{c D} \geq 3$, due to weaker performance for $F_{c D} \leq 3$.

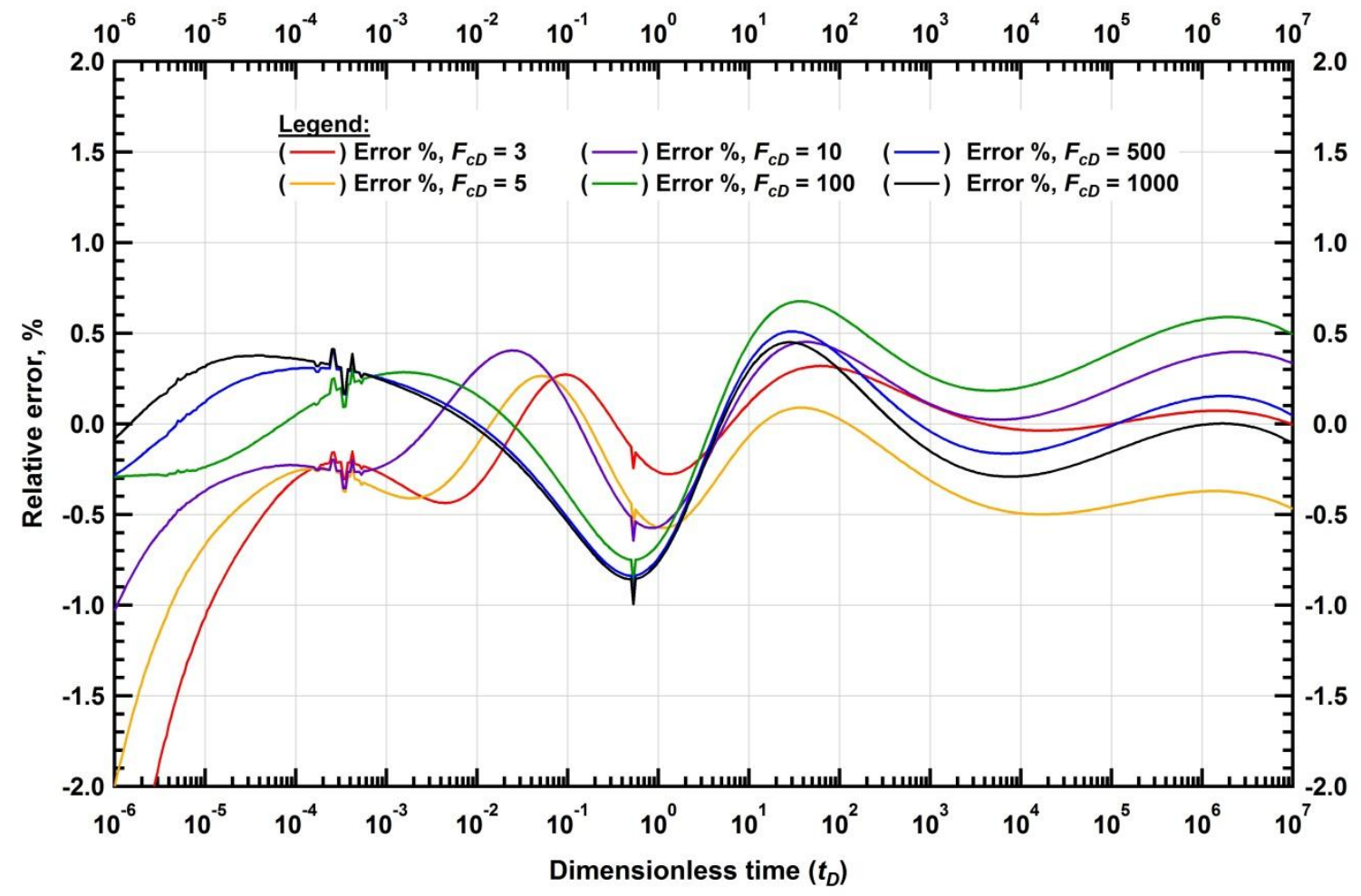

Figure 5.21 - (Scenario 3) Relative error (percent) in the dimensionless pressure solutions versus dimensionless time. 


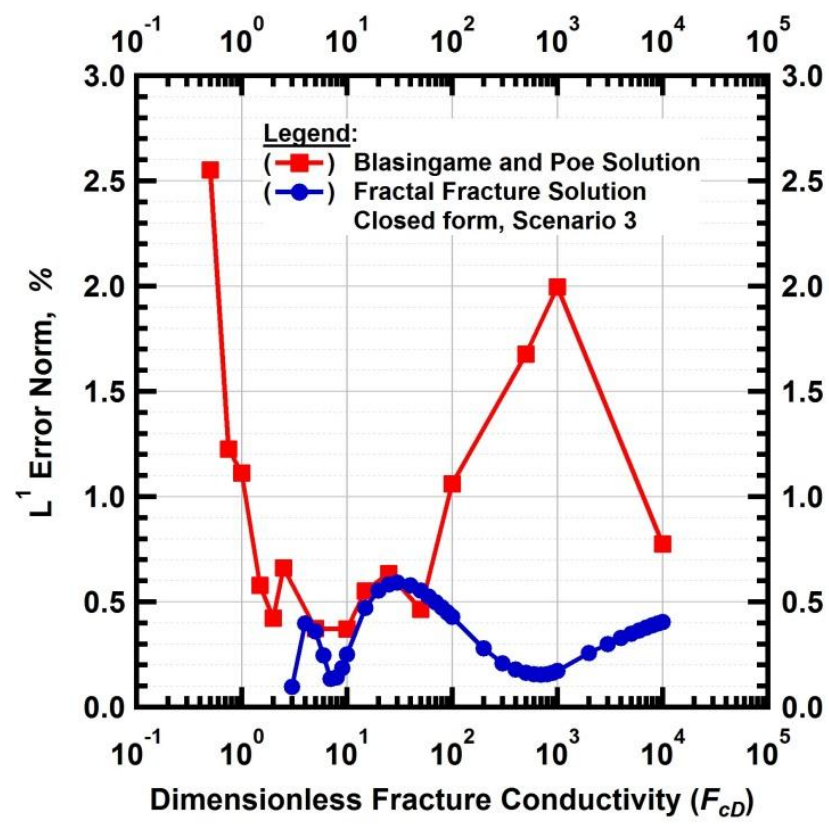

Figure 5.22 - (Scenario 3) $\mathrm{L}^{1}$ relative error norms for the dimensionless pressure solutions for the closed form Fractal-Fracture Solution (FFS) and the Trilinear Pseudoradial Solution (TPRS) (Blasingame and Poe 1993) versus the dimensionless fracture conductivity

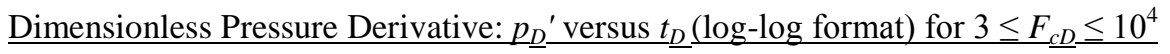

In Figure 5.23 we present the $p_{D}{ }^{\prime}$ versus $t_{D}$ functions for FFS (Scenario 3) and the Cinco-Meng reference solution in log-log format (13 log cycles are shown, this is 13 orders of magnitude in $\left.t_{D}\right)$. As with the $p_{D}$ function for this case, the comparison appears to be near-perfect, there are no visible discrepancies in the solutions.

Dimensionless Pressure Derivative: $\underline{p}_{\underline{D}}{ }^{\prime}$ versus $t_{\underline{D}}$ (semi-log $(x$-axis $)$ format) for $3 \leq F_{c \underline{D}} \leq 10^{4}$

The $p_{D}{ }^{\prime}$ versus $t_{D}$ functions for FFS (Scenario 3) and the Cinco-Meng reference solution in semi-log $(x-$ axis) format (13 log cycles in $\left.t_{D}\right)$ are shown in Figure 5.24. We note that the behavior of the $p_{D}{ }^{\prime}$ function for the range $10^{-6} \leq t_{D} \leq 1$ is excellent, essentially no deviations/discrepancies in the FFS and Cinco-Meng solutions. However, for $t_{D} \geq 1$ we note subtle (but consistent) oscillations in the $p_{D}{ }^{\prime}$ function - which suggests that these oscillations (observed for both Scenarios 2 and 3) are likely artifacts of using the fractal diffusivity equation for a "non-fractal" process. That is, the oscillations are inherent features of the FFS 
method. However, the minimal nature of these oscillatory behaviors suggests that the FFS approach (particularly Scenario 3) is sufficient for practical applications.

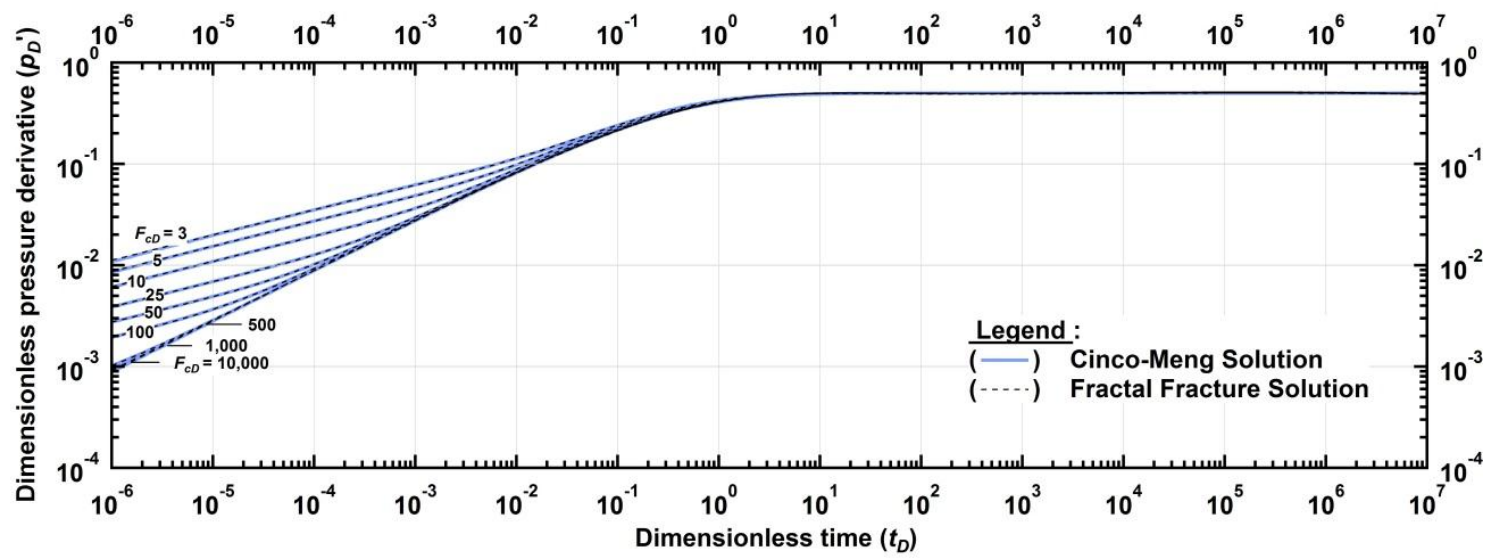

Figure 5.23 - (Scenario 3) Log-log plot of dimensionless pressure derivative function versus dimensionless time for the FFS and Cinco-Meng Solutions (1988).

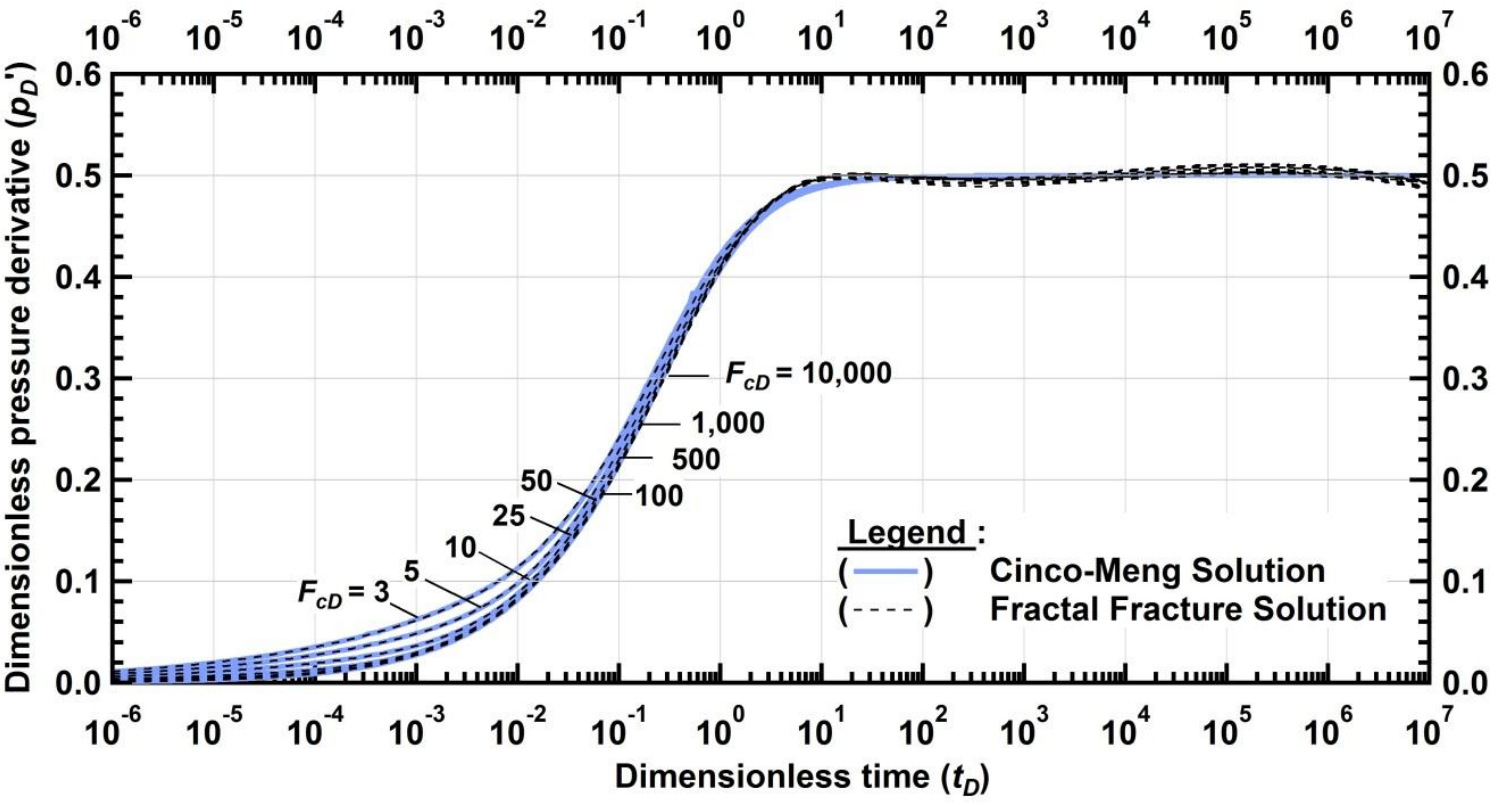

Figure 5.24 - (Scenario 3) Semi-log plot of dimensionless pressure derivative versus dimensionless time for the FFS and Cinco-Meng (1988) Solutions. 
Dimensionless Pressure Derivative: $p_{\underline{D}}{ }^{\prime}$ relative error versus $t_{\underline{D}}($ semi- $\log (x$-axis $))$ for $3<F_{\underline{c D}}<10^{3}$

The relative error for the $p_{D}{ }^{\prime}$ function is presented in Figure 5.25 (for $3<F_{c D}<10^{3}$ ). In Figure 5.25 the oscillations are similar in frequency as those observed in Figure 5.11 (for Scenario 2), but in Figure 5.25 the oscillations vary less (between -2 and 2 percent), suggesting that Scenario 3 is a much better "fit" by comparison to the Cinco-Meng reference solution.

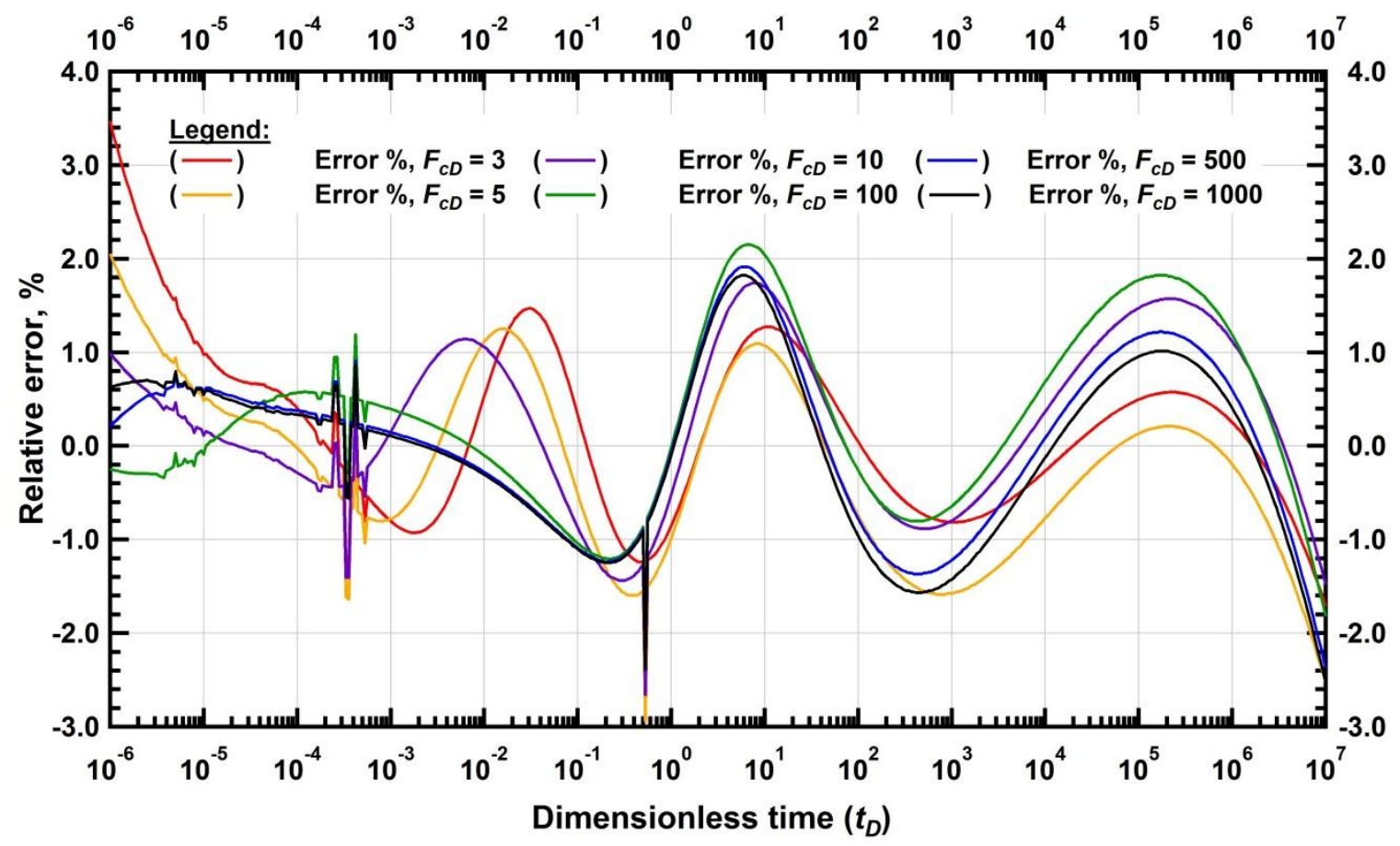

Figure 5.25 - (Scenario 3) Relative error (percent) in the dimensionless pressure derivative solutions versus dimensionless time. 
Dimensionless Pressure Derivative: $\underline{p}_{\underline{D}}{ }^{\prime} \mathrm{L}^{1}$ error norm versus $t_{\underline{D}}$ (semi-log $(x$-axis) $)$ for $3 \leq F_{\underline{c} \underline{D}} \leq 10^{4}$

In Figure 5.26 we present the $\mathrm{L}^{1}$ error norm for the $p_{D}{ }^{\prime}$ function (for $3 \leq F_{c D} \leq 10^{4}$ ) versus the dimensionless fracture conductivity $\left(F_{c D}\right)$, for the Fractal-Fracture Solution (FFS) and the Trilinear Pseudoradial Solution (TPRS) (Blasingame and Poe 1993). From our observations in Figure 5.26, we conclude that the performance of the $p_{D}{ }^{\prime}$ function is very similar for the FFS and TPRS methods, and that the $\mathrm{L}^{1}$ error norm suggests that these solutions should be considered essentially the same (certainly so for practical purposes). As a reminder, we elected not to consider cases for the FFS approach (Scenario 3) where $F_{c D} \leq$ 3 due to weak performance for those cases.

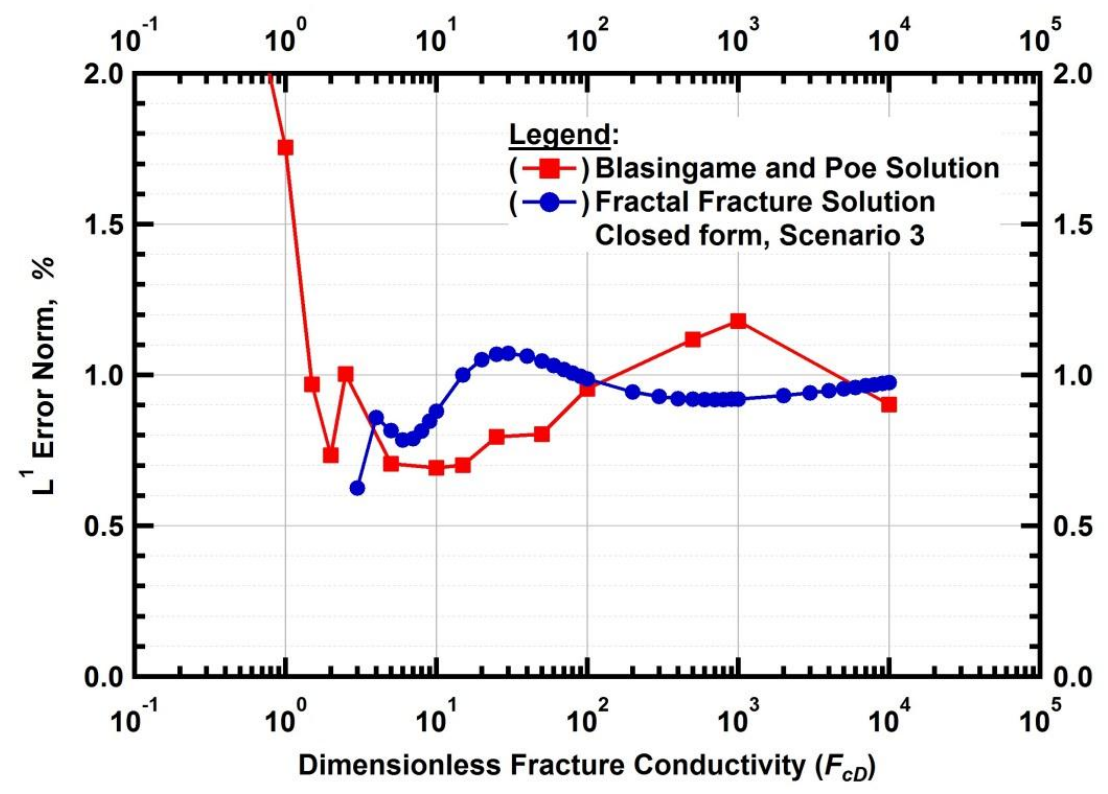

Figure 5.26 - (Scenario 3) $\mathrm{L}^{1}$ relative error norms for the dimensionless pressure derivative solutions for the closed form Fractal-Fracture Solution (FFS) and the Trilinear Pseudoradial Solution (TPRS) (Blasingame and Poe 1993) versus the dimensionless fracture conductivity $\left(F_{c D}\right)$. Reference solution obtained from Cinco-Meng (1988) 


\section{SUMMARY AND CONCLUSIONS}

\subsection{Summary}

1. A rigorous analytical study of the Fractal Diffusivity Equation (Eq. 3.33) was performed, beginning with the Fractal Porosity-Permeability Relations (Eqs. 3.1 and 3.2) - the physical meaning of these relations is discussed, showing why these relations have been largely ignored in the literature.

2. A rigorous analytical and numerical study of the Fractal Diffusivity Equation was performed in Sections 3.3 and 3.4, respectively.

3. In this work we combined the Fractal Diffusivity Equation with the Trilinear Flow Solution originally proposed by Lee and Brockenbrough (1986) (see Figure 4.1). We have called this new solution the "Fractal-Fracture Solution". We replaced the three original linear diffusivity equations of Regions 1, 2 and 3 with the fractal counterparts for these regions. In doing so, 6 unknowns were introduced to the problem - namely the fractal parameters $d_{1}, d_{2}, d_{3}, \theta_{1}, \theta_{2}$ and $\theta_{3}$. We validated this new solution by matching the Cinco-Meng (1988) semi-analytical solution for a single vertical hydraulic fracture.

4. Since it is not obvious what values these parameters should have, we coupled the Fractal-Fracture Solution (FFS) with the Levenberg-Marquardt numerical optimization algorithm (Figure 4.5). We considered three different scenarios; where all three scenarios were successfully matched to the target solution (Figure 4.8), albeit with varying degrees of accuracy.

5. The correlation parameters were generally well behaved (see Figure 4.9, Figure 4.10 and Figure 4.11) and relationships were developed with respect to dimensionless fracture conductivity $F_{c D}$ (Section 5.1 and 5.3). Lastly, these parameters led to two closed-form Fractal-Fracture Solution, one derived from Scenario 2 (Section 5.2) and one derived from Scenario 3 (Section 5.4). 


\subsection{Conclusions}

1. Depending on the value of the fractal parameters $d$ and $\theta$ chosen, it is possible to obtain both the classic linear and radial flow solutions from the Fractal Diffusivity Equation.

2. The pressure signal predicted by the analytical solution for the Fractal Diffusivity Equation was successfully matched via numerical simulation.

3. FFS-Sc2 performance is globally superior to the Blasingame and Poe (1993) Trilinear Pseudoradial Solution in terms of pressure (Figure 5.8) accuracy, but falls a somewhat short in terms of pressure derivative (Figure 5.12) accuracy. We do not recommend using the Fractal-Fracture Solution for values $F_{c D}<0.5$. The FFS-Sc3 has a better pressure derivative behavior than FFS-Sc2 (Figure 5.26), but is only valid for $F_{c D} \geq 3$.

4. For what we believe is the first time, fractal theory has been used in reservoir engineering to address a problem that is not related to naturally-fractured reservoirs or heterogeneous media. We have showed that the Fractal Diffusivity Equation may have untapped potential due to its flexibility in describing a multitude of flow regimes. We believe that this approach may lead to solutions for intractable problems such as multiple transverse fractures, sorption effects or formation damage.

\subsection{Recommendations for Future Research}

\section{General remarks on the physical meaning of the FFS}

In Figure 3.1 we presented a case where two reservoirs in different coordinate systems with different hydraulic properties produce identical pressure signals. Similarly, the Fractal-Fracture Solution (FFS) and the Cinco-Meng Solution (1988) produce nearly identical pressure signals, even though the way the fracture is modeled in each case is vastly different. As such, we have presented two examples where it is possible to create "equivalent flow systems" with relative ease due to the flexibility of fractal theory. 
If $d=2$ implies linear flow, and $d=3$ implies radial flow, what does it mean if $d$ is equal to, say, 2.5? Is this the equivalent of elliptical flow? If so, what does it look like? At present we do not have answers to these questions, but we intuitively suspect a relationship between the fractal parameters $\{d, \theta\}$ and the shape of the pressure waves and/or streamlines.

\section{$\underline{\text { Possible future research directions }}$}

During the investigation it was realized that the fractal parameters 'bend' the pressure curve in different ways, hence this procedure should have great flexibility. In this work we use the classic Cinco-Meng (1988) solution to calibrate/validate our approach, and we note that at present, a basis solution (analytical, semi-analytical, numerical) must be available to calibrate any proposed solution which is based on the generalized Fractal Diffusivity Equation.

Possible problems of interest include reservoirs with sorption effects, wells with formation damage, naturally-fractured reservoirs, and wells with multiple transverse fractures. Table 4.2 contains the analytical solutions for other models should the user want to perform his/her own calibrations with a different Scenario. Should a match be successful, one must be careful not to reduce the problem to a curve-fitting exercise as there is no substitute for understanding the physics of the problem at its root. 


\section{NOMENCLATURE}

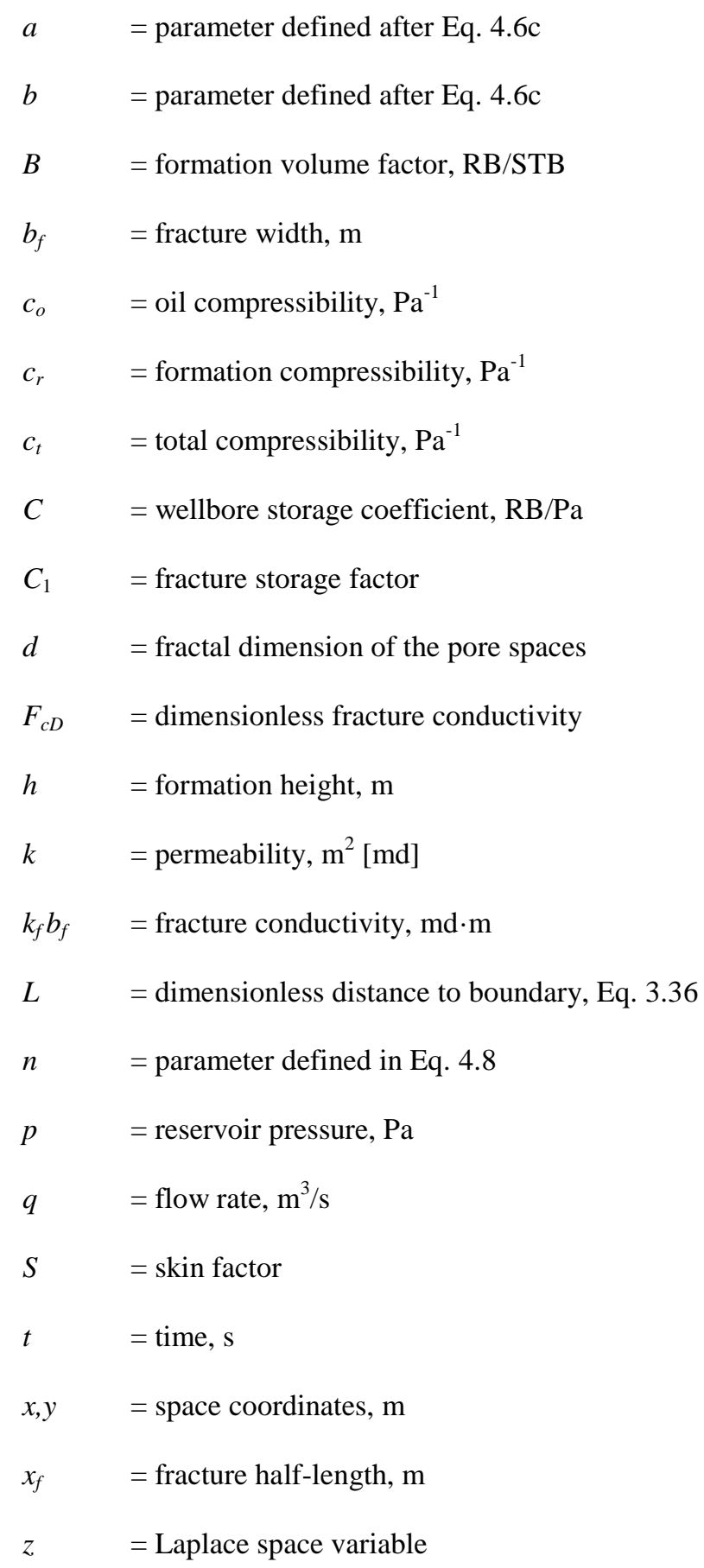




\section{Greek Symbols}

$$
\begin{array}{ll}
\alpha & =\text { parameter defined in Eq. } 4.8 \\
\gamma & =\text { parameter defined in Eq. } 4.8 \\
\theta & =\text { fractal dimension of the fluid flow } \\
\mu & =\text { viscosity, Pa.s [cp] } \\
\phi & =\text { porosity, fraction } \\
\psi & =\text { parameter defined in Eq. } 4.20 \\
\Omega & =\text { parameter defined in Eq. } 4.13
\end{array}
$$

\section{Subscripts}

$$
\begin{array}{ll}
D & =\text { dimensionless } \\
f & =\text { related to the fracture } \\
i & =\text { initial condition } \\
o & =\text { oil } \\
w & =\text { at the sandface } \\
1,2,3 & =\text { index of flow region }
\end{array}
$$




\section{REFERENCES}

Abdassah, D. and Ershaghi, I. 1986. Triple porosity models for representing naturally-fractured reservoirs. SPE Formation Evaluation 1 (2): 113-127. SPE 13409-PA. http://dx.doi.org/10.2118/13409$\underline{\text { PA }}$

Acuña, J.A., Ershaghi, I., and Yortsos, Y.S. 1995. Practical Application of Fractal Pressure-Transient Analysis in Naturally-fractured Reservoirs. SPE Formation Evaluation 10 (3): 173-179. SPE 24705-PA. http://dx.doi.org/10.2118/24705-PA

Acuña, J.A. and Yortsos, Y.C. 1991. Numerical Construction and Flow Simulation in Networks of Fractures using Fractal Geometry. Paper SPE 22703-MS presented at the SPE Annual Technical Conference and Exhibition, Dallas, Texas, 6-9 October 1991. http://dx.doi.org/10.2118/22703-MS.

Baihly, J., Laursen, P., Ogrin, J. et al. 2006. Using Microseismic Monitoring and Advanced Stimulation Technology To Understand Fracture Geometry and Eliminate Screenout Problems in the Bossier Sand of East Texas. Paper SPE 102493 presented at the SPE Annual Technical Conference and Exhibition, San Antonio, Texas, USA, 24-27 September 2006. http://dx.doi.org/10.2118/102493-MS.

Bear, J. 1972. Dynamics of Fluids in Porous Media: Dover.Edition. ISBN 0-486-65675-6.

Beier, R.A. 1994. Pressure-Transient Model for a Vertically Fractured Well in a Fractal Reservoir. SPE Formation Evaluation 9 (2): 122-128. SPE 20582-PA. http://dx.doi.org/10.2118/20582-PA

Blasingame, T.A. 2010a. Petroleum Engineering 620 - Fluid Flow in Petroleum Reservoirs Reservoir Flow Solutions - Linear Flow Solutions: Infinite and Finite-Acting Reservoir Cases. Texas A\&M University, Harold Vance Department of Petroleum Engineering.

Blasingame, T.A. 2010b. Petroleum Engineering 620 - Fluid Flow in Petroleum Reservoirs Reservoir Flow Solutions - Solution of the Radial Flow Diffusivity Equation. Texas A\&M University, Harold Vance Department of Petroleum Engineering.

Blasingame, T.A. and Poe, B.D. 1993. Semianalytic Solutions for a Well With a Single FiniteConductivity Vertical Fracture. Paper SPE 26424-MS presented at the SPE Annual Technical Conference and Exhibition, Houston, Texas, 3-6 October. http://dx.doi.org/10.2118/26424-MS.

Bowman, F. 1958. Introduction to Bessel Functions. New York,: Dover Publications.Edition. ISBN 0486604624 .

Camacho-Velazquez, R., Fuentes-Cruz, G., and Vasquez-Cruz, M.A. 2008. Decline-Curve Analysis of Fractured Reservoirs With Fractal Geometry. SPE Reservoir Evaluation \& Engineering 11 (3): 606619. SPE 104009-PA. http://dx.doi.org/10.2118/104009-PA

Carslaw, H.S. and Jaeger, J.C. 1959. Conduction of Heat in Solids: Oxford University Press.Edition. ISBN 978-0-19-853368-9.

Chang, J. and Yortsos, Y. 1990. Pressure Transient Analysis of Fractal Reservoirs. SPE Formation Evaluation 5 (1). SPE 18170-PA. http://dx.doi.org/10.2118/18170-PA

Chapman, S.J. 2008. Fortran 95/2003 for Scientists and Engineers. Boston: McGraw-Hill.Edition 3rd. ISBN 9780073191577 (pbk. alk. paper) 
Cinco-Ley, H. and Meng, H.-Z. 1988. Pressure Transient Analysis of Wells With Finite Conductivity Vertical Fractures in Double Porosity Reservoirs. Paper SPE 18172-MS presented at the SPE Annual Technical Conference and Exhibition, Houston, Texas, 2-5 October 1988. http://dx.doi.org/10.2118/18172-MS.

Cinco-Ley, H., Samaniego, F., and Dominguez, N. 1978. Transient Pressure Behavior for a Well With a Finite-Conductivity Vertical Fracture SPE Journal 18 (4): 253-264. SPE 6014-PA. http://dx.doi.org/10.2118/6014-PA

Dar, V. 2010. New Investment Models Have Operators Targeting Tight Oil And Hybrid Unconventional Reservoirs. The American Oil and Gas Reporter.

Feder, J. 1988. Fractals. Physics of solids and liquids. New York: Plenum Press.Edition. ISBN 0306428512.

Ferrandon, J. 1948. Les lois de l'écoulement de filtration. Le Génie Civile 2 (125): 24-28.

Flamenco-Lopez, F. and Camacho-Velazquez, R. 2003. Determination of Fractal Parameters of Fracture Networks Using Pressure-Transient Data. SPE Reservoir Evaluation \& Engineering 6 (1). SPE 82607-PA. http://dx.doi.org/10.2118/82607-PA

Frame, M., Manna, S., and Novak, M. 2012. World Scientific Publishing Company Journal. Fractals: Complex Geometry, Patterns, and Scaling in Nature and Society. http://www.worldscinet.com/fractals/mkt/aims_scope.shtml

Friedrich, R. and Gurevich, S. 2010. Numerische Methoden für komplexe Systeme Diffusionsgleichung. Institut für Theoretische Physik - Westfälische Wilhelms-Universität Münster, Germany. http://pauli.uni-muenster.de/tp/en/menu/teaching/archiv/numerische-methoden-0910.html

Fuentes-Cruz, G., Camacho-Velázquez, R., and Vásquez-Cruz, M. 2010. A Unified Approach for Falloff and Buildup Tests Analysis Following a Short Injection/Production Time. Paper SPE 133539-MS presented at the SPE Western Regional Meeting, Anaheim, California, USA, 27-29 May 2010. http://dx.doi.org/10.2118/133539-MS.

Gringarten, A.C., Henry J. Ramey, J., and Raghavan, R. 1974. Unsteady-State Pressure Distributions Created by a Well With a Single Infinite-Conductivity Vertical Fracture. SPE Journal 14 (4). SPE 4051PA. http://dx.doi.org/10.2118/4051-PA

Hardy, H.H. and Beier, R.A. 1994. Fractals in reservoir engineering. Singapore ; River Edge, NJ: World Scientific.Edition. ISBN 9810220693.

Katz, A.J. and Thompson, A.H. 1985. Fractal Sandstone Pores - Implications for Conductivity and Pore Formation. Physical Review Letters 54 (12): 1325-1328.

Kong, X.Y., Li, D.L., and Lu, D.T. 2009. Transient pressure analysis in porous and fractured fractal reservoirs. Sci China Ser E-Tech Sci 52 (9): 2700-2708.

Kreyszig, E. 2005. Advanced Engineering Mathematics: Wiley.Edition 9. ISBN 0471488852.

Krohn, C.E. and Thompson, A.H. 1986. Fractal Sandstone Pores - Automated Measurements Using Scanning-Electron-Microscope Images. Physical Review B 33 (9): 6366-6374.

Lee, J. and Wattenbarger, R.A. 1996. Gas reservoir engineering. SPE textbook series. Richardson, TX: Henry L. Doherty Memorial Fund of AIME, Society of Petroleum Engineers.Edition 1. ISBN 1555630731. 
Lee, S.-T. and Brockenbrough, J.R. 1986. A New Approximate Analytic Solution for FiniteConductivity Vertical Fractures. SPE Formation Evaluation 1 (1). SPE 12013-PA. http://dx.doi.org/10.2118/12013-PA

Maloy, K.J., Boger, F., Feder, J. et al. 1987. Dynamics of Viscous-Fingering Fractals in PorousMedia. Physical Review A 36 (1): 318-324.

Maloy, K.J., Feder, J., and Jossang, T. 1985. Viscous Fingering Fractals in Porous-Media. Physical Review Letters 55 (24): 2688-2691.

Mandelbrot, B. 1967. How Long Is the Coast of Britain? Statistical Self-Similarity and Fractional Dimension. Science 156 (3775): 636-638. http://dx.doi.org/10.1126/science.156.3775.636

Mandelbrot, B.B. 1982. The fractal geometry of nature. San Francisco: W.H. Freeman.Edition. ISBN 0716711869.

McNally, J. 2010. Earth's Most Stunning Natural Fractal Patterns. Wired Magazine. http://www.wired.com/wiredscience/2010/09/fractal-patterns-in-nature/?pid=162. Accessed February 22nd 2012.

Moran, C. 2010. Fractal Coast. http://www.flickr.com/photos/buggs_moran/4516938146/. Accessed February 22nd 2012.

Moré, J.J., Garbow, B.S., and Hillstrom, K.E. 1984. The MINPACK Project. In Sources and Development of Mathematical Software (Prentice-Hall Series in Computational Mathematics), ed. W. J. Cowell, E.

Muñoz, A., Ehlig-Economides, C., and Economides, M.J. 1998. Principal Permeability Determination from Multiple Horizontal Well Tests. Paper SPE 50396-MS presented at the SPE International Conference on Horizontal Well Technology, Calgary, Alberta, Canada, 1-4 November 1998. http://dx.doi.org/10.2118/50396-MS.

Nagel, N.B., Sanchez-Nagel, M., and Lee, B. 2012. Gas Shale Hydraulic Fracturing: A Numerical Evaluation of the Effect of Geomechanical Parameters. Paper 152192 presented at the SPE Hydraulic Fracturing Technology Conference, The Woodlands, Texas, 6-8 February 2012. http://dx.doi.org/10.2118/152192-MS.

Ozkan, E. and Raghavan, R. 1991. New Solutions for Well-Test-Analysis Problems: Part 1 Analytical Considerations SPE Formation Evaluation $6 \quad$ (3). SPE 18615-PA. http://dx.doi.org/10.2118/18615-PA

Ruis, J. 2008. Pore Fractal. Fractal.org. http://www.fractal.org/Life-ScienceTechnology/Publications/Pore-fractal.htm. Accessed February 22nd 2012.

Sheng, J.J. 2010. Discussion of Permeability Anisotropy Effect in Transformation. Journal of Canadian Petroleum Technology 49 (7): 42-46. SPE 139430-PA. http://dx.doi.org/10.2118/139430-PA

Smidt, J.L. and Monro, D.M. 1998. Fractal modeling applied to reservoir characterization and flow simulation. Fractals-Complex Geometry Patterns and Scaling in Nature and Society 6 (4): 401-408. 47-49.

Stehfest, H. 1970. Numerical Inversion of Laplace Transforms. Communications of the ACM 13 (1):

Systat. 2012. TableCurve 2D 5.01. Systat Software, Inc., San Jose, CA. 
Taheri, A., Wessel-Berg, D., Torsæter, O. et al. 2012. The Effects of Anisotropy and Heterogeneity on $\mathrm{CO}_{2}$ Dissolution in Deep Saline Aquifers. Paper SPE 151345-MS presented at the Carbon Management Technology Conference, Orlando, Florida, USA, 7-9 February 2012. http://dx.doi.org/10.7122/151345MS.

US DOE EIA. 2011. Annual Energy Outlook 2011 with Projections to 2035. http://www.eia.gov/forecasts/aeo/pdf/0383\%282011\%29.pdf

Wold, M.B. and Jeffrey, R.G. 1999. A Comparison of Coal Seam Directional Permeability as Measured in Laboratory Core Tests and in Well Interference Tests. Paper SPE 55598-MS presented at the SPE Rocky Mountain Regional Meeting, Gillette, Wyoming, 15-18 May 1999. http://dx.doi.org/10.2118/55598-MS.

Wolfram. 2010. Mathematica: Version 8.0. Wolfram Research, Inc., Champaign, Illinois.

Yu, B. 2008. Analysis of Flow in Fractal Porous Media. Applied Mechanics Reviews 61 (5): 050801105080119.

Yun, M.J., Yu, B.M., and Cai, J.C. 2009. Analysis of seepage characters in fractal porous media. International Journal of Heat and Mass Transfer $52 \quad$ (14): 3272-3278. http://dx.doi.org/10.1016/j.ijheatmasstransfer.2009.01.024 


\section{APPENDIX A}

Table A.1 - Tabular results from Scenario 2

\begin{tabular}{|c|c|c|c|c|c|c|}
\hline$F_{c D}$ & $\begin{array}{c}d_{3} \\
\text { optimized }\end{array}$ & $\begin{array}{c}\theta_{3} \\
\text { optimized }\end{array}$ & $\begin{array}{c}d_{2} \\
\text { optimized }\end{array}$ & $\begin{array}{c}\theta_{2} \\
\text { optimized }\end{array}$ & $\begin{array}{c}\text { FFS_Sc2_closed } \\
p_{D} \text { norm error }\end{array}$ & $\begin{array}{c}\text { FFS_Sc2_closed } \\
p_{D}{ }^{\prime} \text { norm error }\end{array}$ \\
\hline 0.5 & 1.258 & -1.611 & 2.001 & -0.7022 & 2.148 & 4.12 \\
\hline 0.6 & 1.3 & -1.587 & 2.014 & -0.51 & 0.45 & 1.482 \\
\hline 0.7 & 1.413 & -1.453 & 1.95 & -0.433 & 0.543 & 1.433 \\
\hline 0.8 & 1.566 & -1.264 & 1.864 & -0.39 & 0.643 & 1.432 \\
\hline 0.9 & 1.709 & -1.091 & 1.79 & -0.361 & 0.615 & 1.347 \\
\hline 1 & 1.839 & -0.9352 & 1.728 & -0.34 & 0.557 & 1.323 \\
\hline 1.5 & 2.263 & -0.428 & 1.548 & -0.295 & 0.4 & 1.584 \\
\hline 2 & 2.45 & -0.203 & 1.479 & -0.282 & 0.422 & 1.805 \\
\hline 3 & 2.551 & -0.0769 & 1.447 & -0.279 & 0.405 & 1.912 \\
\hline 4 & 2.515 & -0.1195 & 1.461 & -0.281 & 0.35 & 1.866 \\
\hline 5 & 2.47 & -0.171 & 1.477 & -0.285 & 0.316 & 1.804 \\
\hline 6 & 2.412 & -0.2415 & 1.497 & -0.289 & 0.324 & 1.75 \\
\hline 7 & 2.369 & -0.2922 & 1.512 & -0.292 & 0.368 & 1.706 \\
\hline 8 & 2.344 & -0.322 & 1.522 & -0.294 & 0.401 & 1.668 \\
\hline 9 & 2.313 & -0.3594 & 1.533 & -0.296 & 0.423 & 1.636 \\
\hline 10 & 2.289 & -0.3876 & 1.542 & -0.298 & 0.437 & 1.607 \\
\hline 20 & 2.145 & -0.5623 & 1.594 & -0.309 & 0.442 & 1.425 \\
\hline 30 & 2.091 & -0.628 & 1.614 & -0.313 & 0.403 & 1.334 \\
\hline 40 & 2.061 & -0.6649 & 1.625 & -0.315 & 0.37 & 1.279 \\
\hline 50 & 2.045 & -0.6846 & 1.632 & -0.317 & 0.345 & 1.244 \\
\hline 60 & 2.031 & -0.7012 & 1.637 & -0.318 & 0.325 & 1.22 \\
\hline 70 & 2.023 & -0.7117 & 1.64 & -0.319 & 0.309 & 1.202 \\
\hline 80 & 2.017 & -0.7192 & 1.642 & -0.319 & 0.295 & 1.188 \\
\hline 90 & 2.011 & -0.7257 & 1.644 & -0.319 & 0.284 & 1.178 \\
\hline 100 & 2.008 & -0.7295 & 1.646 & -0.32 & 0.275 & 1.169 \\
\hline 200 & 1.987 & -0.7558 & 1.654 & -0.321 & 0.228 & 1.131 \\
\hline 300 & 1.984 & -0.7599 & 1.655 & -0.322 & 0.217 & 1.119 \\
\hline 400 & 1.978 & -0.7667 & 1.657 & -0.322 & 0.213 & 1.113 \\
\hline 500 & 1.977 & -0.7675 & 1.658 & -0.322 & 0.211 & 1.109 \\
\hline 600 & 1.975 & -0.7704 & 1.659 & -0.322 & 0.21 & 1.107 \\
\hline 700 & 1.974 & -0.7715 & 1.659 & -0.322 & 0.21 & 1.106 \\
\hline 800 & 1.973 & -0.7725 & 1.659 & -0.322 & 0.21 & 1.104 \\
\hline 900 & 1.974 & -0.772 & 1.659 & -0.323 & 0.209 & 1.104 \\
\hline 1000 & 1.974 & -0.7721 & 1.659 & -0.323 & 0.21 & 1.103 \\
\hline 2000 & 1.97 & -0.7761 & 1.66 & -0.323 & 0.213 & 1.101 \\
\hline 3000 & 1.97 & -0.7762 & 1.66 & -0.323 & 0.218 & 1.102 \\
\hline 4000 & 1.97 & -0.7766 & 1.661 & -0.323 & 0.225 & 1.103 \\
\hline 5000 & 1.971 & -0.7752 & 1.66 & -0.323 & 0.232 & 1.105 \\
\hline 6000 & 1.968 & -0.7789 & 1.661 & -0.323 & 0.239 & 1.106 \\
\hline 7000 & 1.969 & -0.7779 & 1.661 & -0.323 & 0.244 & 1.107 \\
\hline 8000 & 1.97 & -0.777 & 1.661 & -0.323 & 0.249 & 1.108 \\
\hline 9000 & 1.969 & -0.7778 & 1.661 & -0.323 & 0.254 & 1.11 \\
\hline 10000 & 1.969 & -0.7778 & 1.661 & -0.323 & 0.258 & 1.111 \\
\hline
\end{tabular}


Table A.2 - Tabular results from Scenario 3

\begin{tabular}{|c|c|c|c|c|c|c|c|c|}
\hline$F c D$ & $\begin{array}{c}d_{3} \\
\text { optimized }\end{array}$ & $\begin{array}{c}\theta_{3} \\
\text { optimized }\end{array}$ & $\begin{array}{c}d_{2} \\
\text { optimized }\end{array}$ & $\begin{array}{c}\theta_{2} \\
\text { optimized }\end{array}$ & $\begin{array}{c}d_{1} \\
\text { optimized }\end{array}$ & $\begin{array}{c}\theta_{1} \\
\text { optimized } \\
\end{array}$ & $\begin{array}{c}\text { FFS_Sc3_closed } \\
p_{D} \text { norm error }\end{array}$ & $\begin{array}{c}\text { FFS_Sc3_closed } \\
p_{D}{ }^{\prime} \text { norm error }\end{array}$ \\
\hline 0.5 & 1.570 & -1.174 & 1.840 & -0.991 & 1.406 & -1.983 & $\mathrm{n} / \mathrm{a}$ & $\mathrm{n} / \mathrm{a}$ \\
\hline 0.6 & 1.573 & -1.168 & 1.774 & -1.099 & 1.443 & -1.662 & $\mathrm{n} / \mathrm{a}$ & $\mathrm{n} / \mathrm{a}$ \\
\hline 0.7 & 1.596 & -1.136 & 1.764 & -1.110 & 1.415 & -1.526 & $\mathrm{n} / \mathrm{a}$ & $\mathrm{n} / \mathrm{a}$ \\
\hline 0.8 & 1.589 & -1.146 & 1.797 & -1.057 & 1.383 & -1.454 & $\mathrm{n} / \mathrm{a}$ & $\mathrm{n} / \mathrm{a}$ \\
\hline 0.9 & 1.581 & -1.157 & 1.820 & -1.015 & 1.364 & -1.398 & $\mathrm{n} / \mathrm{a}$ & $\mathrm{n} / \mathrm{a}$ \\
\hline 1 & 1.570 & -1.175 & 1.835 & -0.988 & 1.357 & -1.345 & $\mathrm{n} / \mathrm{a}$ & $\mathrm{n} / \mathrm{a}$ \\
\hline 1.5 & 1.527 & -1.238 & 1.839 & -0.994 & 1.375 & -1.179 & $\mathrm{n} / \mathrm{a}$ & $\mathrm{n} / \mathrm{a}$ \\
\hline 2 & 1.504 & -1.274 & 1.791 & -1.085 & 1.425 & -1.054 & $\mathrm{n} / \mathrm{a}$ & $\mathrm{n} / \mathrm{a}$ \\
\hline 3 & 1.541 & -1.261 & 1.928 & -0.588 & 1.542 & -0.830 & 0.095 & 0.625 \\
\hline 4 & 1.597 & -1.201 & 1.899 & -0.510 & 1.635 & -0.659 & 0.397 & 0.859 \\
\hline 5 & 1.638 & -1.158 & 1.873 & -0.470 & 1.696 & -0.551 & 0.360 & 0.816 \\
\hline 6 & 1.664 & -1.130 & 1.854 & -0.445 & 1.738 & -0.477 & 0.245 & 0.784 \\
\hline 7 & 1.692 & -1.099 & 1.836 & -0.425 & 1.773 & -0.413 & 0.135 & 0.789 \\
\hline 8 & 1.708 & -1.080 & 1.824 & -0.415 & 1.795 & -0.376 & 0.140 & 0.814 \\
\hline 9 & 1.721 & -1.067 & 1.814 & -0.406 & 1.814 & -0.342 & 0.186 & 0.847 \\
\hline 10 & 1.724 & -1.065 & 1.809 & -0.400 & 1.828 & -0.319 & 0.251 & 0.880 \\
\hline 20 & 1.777 & -1.006 & 1.768 & -0.369 & 1.899 & -0.196 & 0.553 & 1.051 \\
\hline 30 & 1.789 & -0.993 & 1.756 & -0.360 & 1.922 & -0.158 & 0.591 & 1.072 \\
\hline 40 & 1.805 & -0.975 & 1.746 & -0.354 & 1.935 & -0.135 & 0.578 & 1.062 \\
\hline 50 & 1.805 & -0.976 & 1.744 & -0.352 & 1.942 & -0.123 & 0.553 & 1.046 \\
\hline 60 & 1.817 & -0.961 & 1.737 & -0.350 & 1.948 & -0.114 & 0.525 & 1.031 \\
\hline 70 & 1.812 & -0.968 & 1.738 & -0.349 & 1.951 & -0.111 & 0.498 & 1.018 \\
\hline 80 & 1.815 & -0.965 & 1.736 & -0.347 & 1.954 & -0.107 & 0.473 & 1.006 \\
\hline 90 & 1.820 & -0.958 & 1.733 & -0.346 & 1.957 & -0.103 & 0.449 & 0.996 \\
\hline 100 & 1.819 & -0.961 & 1.733 & -0.346 & 1.958 & -0.103 & 0.428 & 0.987 \\
\hline 200 & 1.827 & -0.951 & 1.727 & -0.342 & 1.967 & -0.105 & 0.279 & 0.943 \\
\hline 300 & 1.831 & -0.947 & 1.725 & -0.341 & 1.970 & -0.104 & 0.208 & 0.928 \\
\hline 400 & 1.837 & -0.939 & 1.722 & -0.340 & 1.971 & -0.104 & 0.179 & 0.921 \\
\hline 500 & 1.838 & -0.938 & 1.721 & -0.340 & 1.973 & -0.104 & 0.164 & 0.919 \\
\hline 600 & 1.839 & -0.937 & 1.720 & -0.340 & 1.973 & -0.104 & 0.156 & 0.918 \\
\hline 700 & 1.840 & -0.936 & 1.720 & -0.339 & 1.974 & -0.104 & 0.154 & 0.918 \\
\hline 800 & 1.833 & -0.945 & 1.722 & -0.339 & 1.974 & -0.104 & 0.157 & 0.918 \\
\hline 900 & 1.828 & -0.952 & 1.724 & -0.339 & 1.974 & -0.104 & 0.162 & 0.919 \\
\hline 1000 & 1.842 & -0.934 & 1.718 & -0.338 & 1.976 & -0.104 & 0.171 & 0.920 \\
\hline 2000 & 1.851 & -0.923 & 1.714 & -0.337 & 1.978 & -0.104 & 0.256 & 0.932 \\
\hline 3000 & 1.851 & -0.923 & 1.714 & -0.337 & 1.978 & -0.104 & 0.299 & 0.941 \\
\hline 4000 & 1.851 & -0.923 & 1.714 & -0.337 & 1.978 & -0.104 & 0.328 & 0.948 \\
\hline 5000 & 1.851 & -0.923 & 1.714 & -0.337 & 1.978 & -0.104 & 0.348 & 0.954 \\
\hline 6000 & 1.851 & -0.923 & 1.714 & -0.337 & 1.979 & -0.104 & 0.364 & 0.959 \\
\hline 7000 & 1.846 & -0.930 & 1.716 & -0.337 & 1.978 & -0.104 & 0.377 & 0.964 \\
\hline 8000 & 1.851 & -0.923 & 1.714 & -0.337 & 1.979 & -0.104 & 0.388 & 0.967 \\
\hline 9000 & 1.851 & -0.923 & 1.714 & -0.337 & 1.979 & -0.104 & 0.397 & 0.971 \\
\hline 10000 & 1.851 & -0.923 & 1.714 & -0.337 & 1.979 & -0.104 & 0.405 & 0.974 \\
\hline
\end{tabular}




\section{APPENDIX B}

In this Appendix we show the FORTRAN2003 code for the closed-form Fractal-Fracture Solution

(Scenario 2). The following is the code for the file containing the driver.

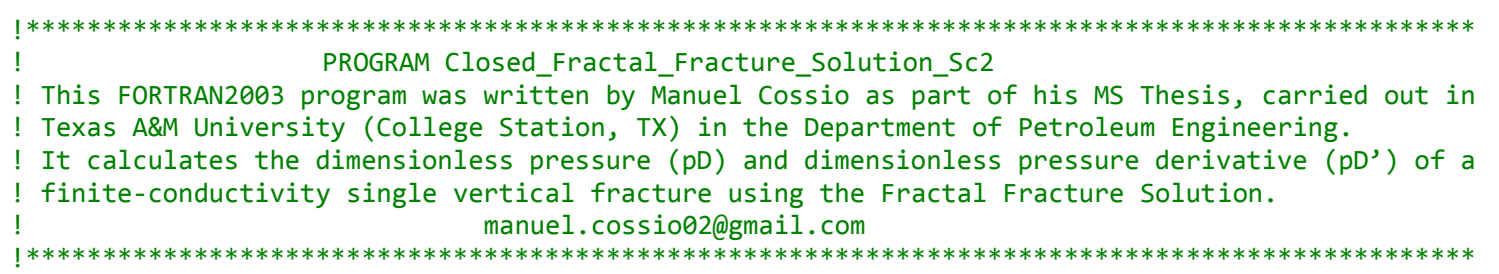

PROGRAM Closed_Fractal_Fracture_Solution_Sc2

USE Aux_Functions ! This module contains the Auxiliary functions

USE Precision ! This module contains the precision integer DP, in this case set to Quad Precision

IMPLICIT NONE

$\begin{array}{ll}\text { INTEGER } & :: \text { sizetime } \\ \text { INTEGER } & :: \text {, j } \\ \text { INTEGER } & :: \text { ngavmax } \\ \text { REAL (DP) } & :: \text { gstehfest_sum, gstehfest_sum_der, starttimer, stoptimer } \\ \text { REAL (DP) } & :: t, d 3, z i, \text { theta3, d2, theta2, FCD, tDmin, tDmax, tDinc, expi } \\ \text { REAL (DP), ALLOCATABLE, DIMENSION(:) :: tD, pwD, pwDder }\end{array}$

! Use the Fortran intrinsic function CPU_TIME to measure how long calculation takes place CALL CPU_TIME(starttimer)

! Read input file using Namelists NAMELIST/CFFS_input/ FCD, tDmin, tDmax, sizetime, ngavmax

! Call Subroutine "Estimate_Fractal_Params" to obtain values of fractal parameters from correlations discussed in Section 5.1

! INPUT: FCD

! OUTPUT: d3, theta3, d2, theta2

CALL Estimate_Fractal_Params(FcD, d3, theta3, d2, theta2)

! Write in the Output file the fracture conductivity and its corresponding fractal parameters $\operatorname{WRITE}(*, 11) \mathrm{FcD}, \mathrm{d} 3$, theta3, d2, theta2 


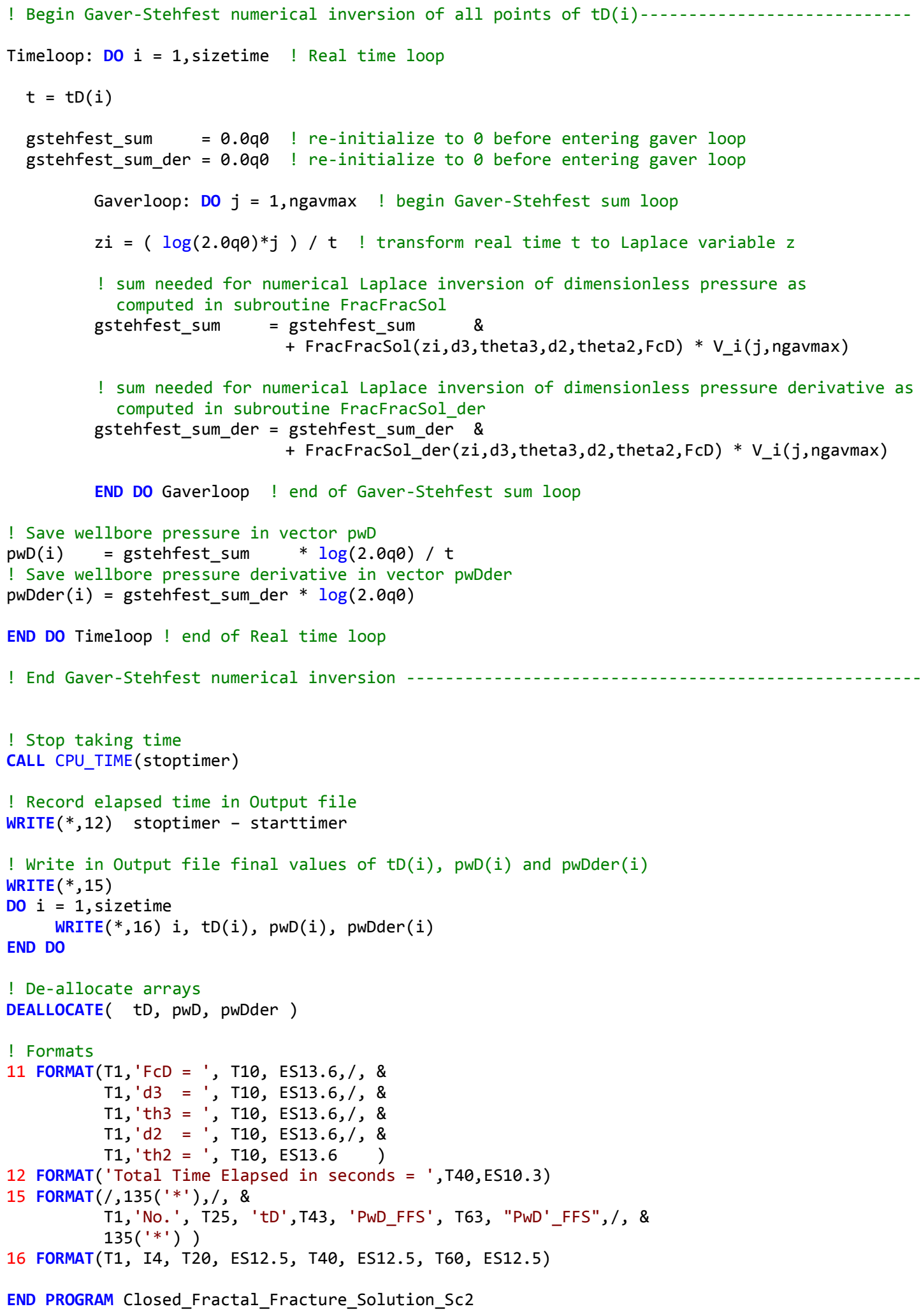


The following code may be placed in a separate file. It shows the additional needed modules.

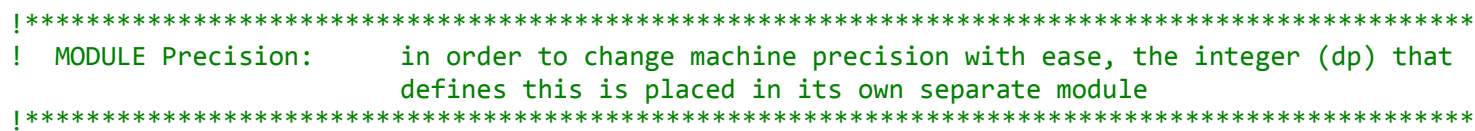

MODULE Precision

INTEGER, PARAMETER : : $d p=\operatorname{KIND}(1.0 \mathrm{Q} 0)$ ! currently in quad precision $Q$

END MODULE Precision

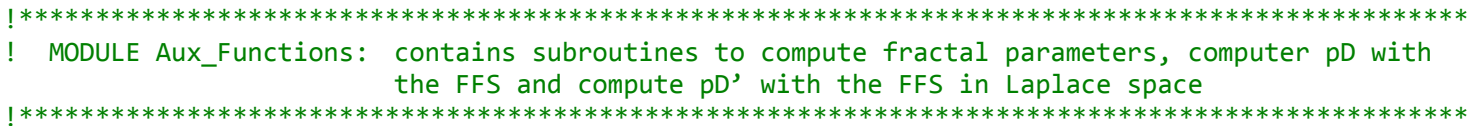

MODULE Aux_Functions

IMPLICIT NONE

PRIVATE

PUBLIC :: Estimate_Fractal_Params, FracFracSol, FracFracSol_der, V_i

\section{CONTAINS}

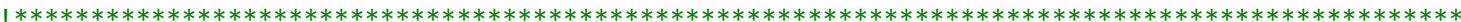

! SUBROUTINE Estimate_Fractal_Params : computes fractal parameters based on correlations as described in Section 5.1

! $* * * * * * * * * * * * * * * * * * * * * * * * * * * * * * * * * * * * * * * * * * * * * * * * * * * * * * * * * * * * * * * * * * * * * * * * * * * * * * * * * * * * * * * * * * * * * * *$

SUBROUTINE Estimate_Fractal_Params(FcD, d3param, theta3param, d2param, theta2param)

USE Precision

REAL (dp), INTENT(IN) : : FCD

REAL (dp), INTENT(OUT) : : d3param, theta3param, d2param, theta2param

REAL (dp) : : A1, B1, C1, D1, E1, F1, G1, $x$

REAL (dp) : : A2, B2, C2, D2, E2, F2, G2

$X=\operatorname{LOG10}(\mathrm{FCD})$

! Coefficients for correlations

$\mathrm{A} 1=1.732300052 \mathrm{~d} 0$

$\mathrm{B1}=0.145343775 \mathrm{d \theta}$

$\mathrm{C} 1=-1.00458904 \mathrm{~d} \theta$

$\mathrm{D} 1=2.270990983 \mathrm{~d} \theta$

$E 1=4.547075660 \mathrm{~d} \theta$

$\mathrm{F} 1=1.016405890 \mathrm{d \theta}$

$\mathrm{G} 1=1.566926538 \mathrm{~d} \theta$

$A 2=-0.34048432 \mathrm{~d} 0$

$B 2=1.918772436 \mathrm{d \theta}$

$C 2=-0.26952048 \mathrm{~d} 0$

$\mathrm{D} 2=0.433916281 \mathrm{~d} \theta$

$E 2=-0.34553019 \mathrm{~d} 0$ 


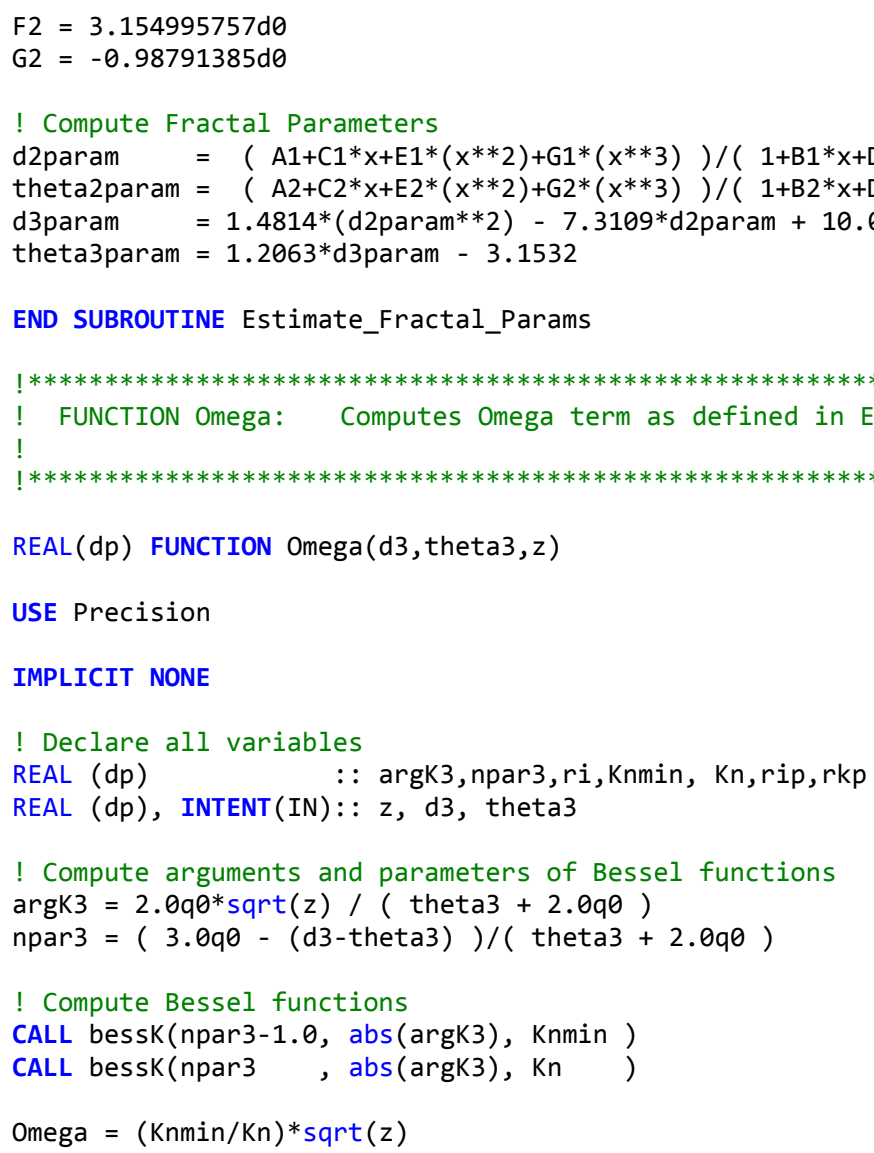

\section{IMPLICIT NONE}

! Declare all variables

REAL (dp), PARAMETER :: Pi = 3.141592653589793238462643

REAL (dp) :: argK2, npar2, ri, Knmin, Kn, rip, rkp, Kntry

REAL (dp) :: Psi, testpsi

REAL (dp), INTENT(IN):: $z, d 3$, theta3, d2, theta2, FcD

! Compute arguments and parameters of Bessel functions

$\operatorname{argK} 2=2.0 \mathrm{q} \theta^{*} \operatorname{sqrt}(\mathrm{z}+$ Omega $(\mathrm{d} 3$, theta $3, \mathrm{z})) /($ theta $2+2.0 \mathrm{q} \theta)$

npar2 $=(3.0 \mathrm{q} \theta-(\mathrm{d} 2-$ theta 2$)) /($ theta $2+2.0 \mathrm{q} \theta)$

! Compute Bessel functions

CALL bessK( npar2-1.0q0, abs(argK2), Knmin )

CALL bessK( npar2, abs(argK2), Kn )

! Testing to see if there is a problem inside Psi, only for debugging purposes

testpsi $=(2.0 \mathrm{q} \theta * \operatorname{SQRT}(\mathrm{z}+\operatorname{Omega}(\mathrm{d} 3$, theta3,z $)) *(\mathrm{Knmin} / \mathrm{Kn})-$ theta2*npar2 $) / \mathrm{Fcd}$ 


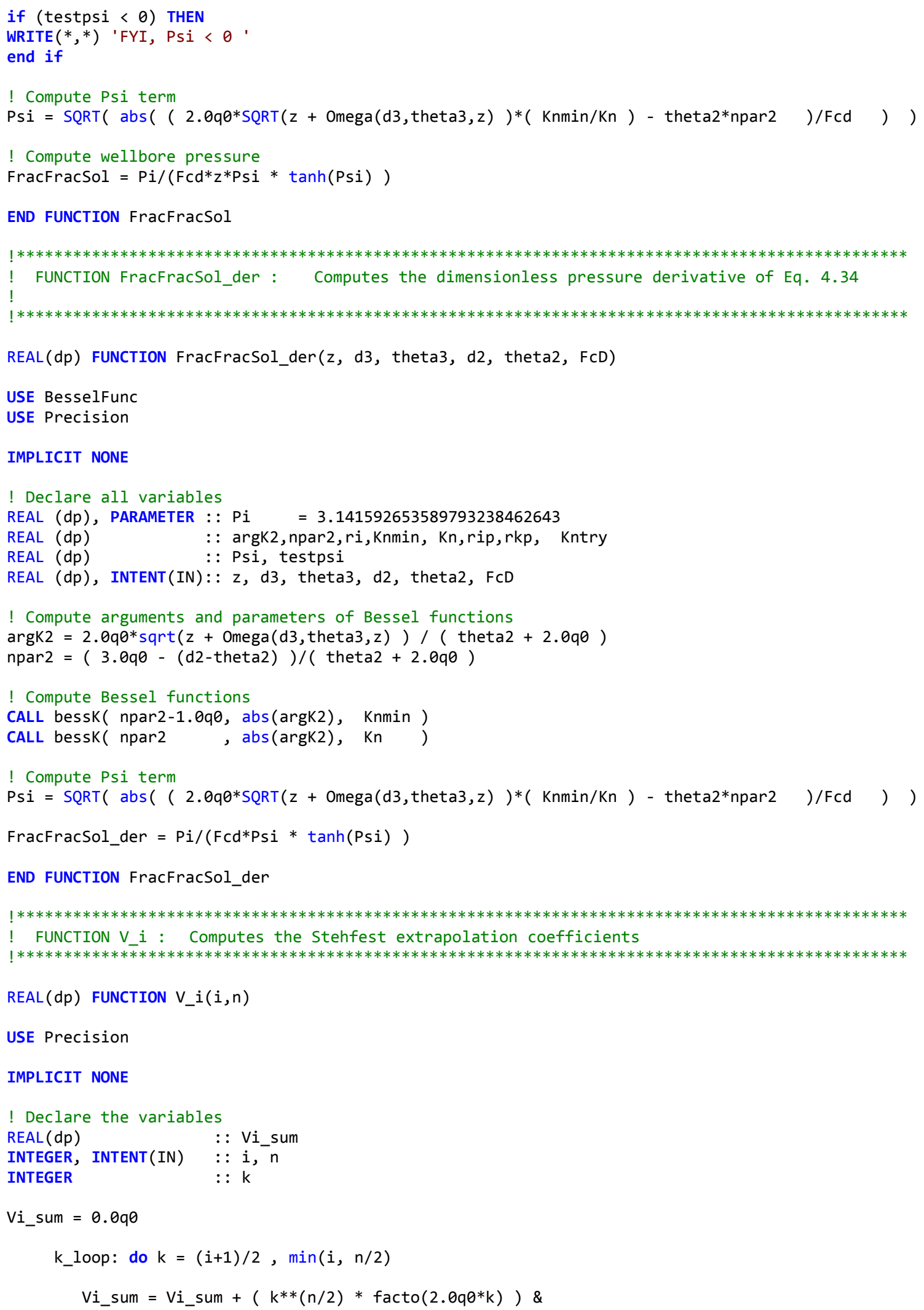




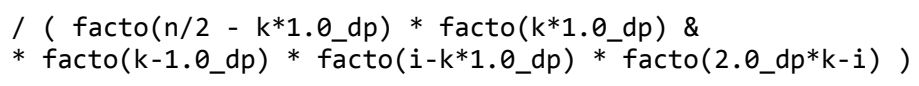

In addition to the three files shown above, the user needs to have a subroutine or function for the computation of modified Bessel functions $I_{n}$ and $K_{n}$, where $n$ is a real number. In this code they are called by the subroutine bess $K$. 


\section{VITA}

Name:

Mailing Address:

E-mail Address:

Education:
Manuel Cossío Santizo

3116 TAMU - 407 Richardson Building

College Station, TX 77843-3116

manuel.cossio02@gmail.com

manuel.cossio02@imperial.ac.uk

manuel.cossio@pe.tamu.edu

Texas A\&M University, College Station, TX, USA

Master of Science Degree in Petroleum Engineering

August 2012

Imperial College London, UK

Master of Engineering Degree in Aeronautical Engineering

June 2006

Society of Petroleum Engineers 